\title{
Fabrication of 3D Conjugated Polymer Structures via Vat Polymerization Additive Manufacturing
}

Andrew T. Cullen, The University of Western Ontario

Supervisor: Price, Aaron D., The University of Western Ontario

A thesis submitted in partial fulfillment of the requirements for the Master of Engineering Science degree in Mechanical and Materials Engineering

(c) Andrew T. Cullen 2018

Follow this and additional works at: https://ir.lib.uwo.ca/etd

Part of the Electrical and Electronics Commons, Electro-Mechanical Systems Commons, Manufacturing Commons, Other Mechanical Engineering Commons, and the Polymer and Organic Materials Commons

\section{Recommended Citation}

Cullen, Andrew T., "Fabrication of 3D Conjugated Polymer Structures via Vat Polymerization Additive Manufacturing" (2018). Electronic Thesis and Dissertation Repository. 5356.

https://ir.lib.uwo.ca/etd/5356

This Dissertation/Thesis is brought to you for free and open access by Scholarship@Western. It has been accepted for inclusion in Electronic Thesis and Dissertation Repository by an authorized administrator of Scholarship@Western. For more information, please contact wlswadmin@uwo.ca. 


\begin{abstract}
Conjugated polymers are a class of electromechanically active materials that can produce motion in response to an electric potential. This motion can be harnessed to perform mechanical work, and therefore these materials are particularly well suited for use as sensors and actuators in microelectromechanical systems. Conventional methods to fabricate conjugated polymer actuators result in planar morphologies that limit fabricated devices to simplistic linear or bending actuation modes. To overcome this limitation, this work develops a conjugated polymer formulation and associated additive manufacturing method capable of realizing three-dimensional conductive polymer structures. A light-based additive manufacturing technique known as vat polymerization is employed due to its ability to fabricate complex microscale features. A specially-formulated photosensitive polypyrrole resin was optimized for the production of microscale 3D structures. The transduction properties of the photosensitive polymer formulation were characterized to evaluate the material's application in mechanical sensing and actuation technologies.
\end{abstract}

Keywords: polypyrrole, conjugated polymers, photopolymerization, additive manufacturing, vat polymerization, 3D printing. 


\section{Acknowledgements}

Financial support for my project was generously provided by the Province of Ontario, NSERC Canada, WORLDiscoveries, and the University of Western Ontario.

Thanks to my Program Advisor, Prof. Liying Jiang for her guidance throughout my project. Thanks to Esstech Inc., IGM Resins Inc., and Chemroy Canada Inc. for the donation of chemicals to support my photopolymer research. Thanks to Dr. Yara Hosein for her assistance with my investigation into the piezoresistive properties of hybrid polypyrrole formulations. Thank you to Dr. Cheryle Séguin for the use of her laboratory's Univert Materials Testing Machine. Thanks to Karen Nygard for her microscopy support. Thanks to Chris Vandelaar for his assistance manufacturing numerous components throughout my project.

Special thanks to my thesis supervisor Prof. Aaron Price for his support and dedication to my project and professional development. Thank you for providing me with the opportunity to interact with global leaders in the field of electroactive polymers, introducing me to many new areas of interest, and being a great role model.

Thank you to my colleagues in the Organic Mechatronics and Smart Materials Laboratory for your insight, support, and friendship throughout my studies. I would like to finally thank my family and friends for their unconditional support in all of my endeavours. 


\section{Contents}

Contents $\quad$ iv

List of Tables viii

List of Figures $\quad$ ix

List of Acronyms and Symbols $\quad$ xiii

1 Introduction 1

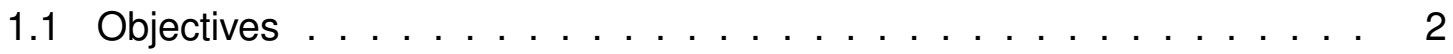

1.2 Major contributions . . . . . . . . . . . . . . 3

1.3 Organization of the thesis . . . . . . . . . . . . . . . 4

List of references . . . . . . . . . . . . . . . . . . . . 5

2 Background and state of the art $\quad 6$

2.1 Review of vat polymerization additive manufacturing technology . . . . . 6

2.2 Review of photopolymerization . . . . . . . . . . . . . . . . . . 14

2.2.1 Photoinitiating system . . . . . . . . . . . . . . . . . . 15

2.2 .2 Oligomers . . . . . . . . . . . . . . . . . 16

2.2 .3 Monomers . . . . . . . . . . . . . . . . . . . . 17

2.2 .4 Light absorber . . . . . . . . . . . . . . . . . . . . . 17

2.2.5 Characterization of photopolymer formulations . . . . . . . . . . 17 
2.3 Review of conjugated polymers . . . . . . . . . . . . . . 18

2.3.1 Photosensitive polypyrrole formulations . . . . . . . . 21

2.4 Chapter summary . . . . . . . . . . . . . . . . . . . 25

List of references . . . . . . . . . . . . . . . . . . . . 25

3 Fabrication system development 31

3.1 Preliminary investigation into vat polymerization of conjugated polymers 32

3.1.1 Fixed-surface vat polymerization . . . . . . . . . . . 32

3.1.2 Free-surface vat polymerization . . . . . . . . 33

3.2 Design of high resolution vat polymerization system $\ldots \ldots \ldots$

3.2.1 Design requirements . . . . . . . . . . . . . . . . 35

3.2.2 Design of DLP light engine setup . . . . . . . . . . . 36

3.2.3 Design of laminating system $\ldots \ldots \ldots$. . . . . . . . 37

3.2.4 Design of multi-material system and build platform . . . . . . 40

3.2 .5 System control . . . . . . . . . . . . . . . . . . 43

3.3 Calibration procedures $\ldots \ldots \ldots \ldots \ldots \ldots$

3.3.1 Build plate levelling procedure $\ldots \ldots \ldots \ldots$

3.3.2 Liquid level measurement . . . . . . . . . . . . . . . . 48

3.3.3 Light engine focal length measurement $\ldots \ldots \ldots \ldots$

3.3.4 Settling time measurement . . . . . . . . . . . . . 5 53

3.4 Fabrication of $3 \mathrm{D}$ microscale structures $\ldots \ldots \ldots \ldots \ldots$

3.5 Chapter summary $\ldots \ldots \ldots \ldots \ldots \ldots \ldots$

List of references . . . . . . . . . . . . . . . 56

4 Development of a photosensitive conjugated polymer formulation $\quad 57$

4.1 Photopolymerization of a pyrrole-silver formulation $\ldots \ldots \ldots$

4.1.1 Design and characterization of a pyrrole-silver formulation . . . 57

4.1 .2 Fixed-surface vat polymerization . . . . . . . . . . . 59 
4.1.3 Free-surface vat polymerization $\ldots \ldots \ldots$. . . . . . 61

4.1.4 Conclusions on the pyrrole-silver formulation . . . . . . . 62

4.2 Photopolymerization of pyrrole-UDMA hybrid polymer formulation . . . 63

4.2.1 Design of pyrrole-UDMA hybrid polymer formulation . . . . 63

4.2.2 Depth of cure . . . . . . . . . . . . . . 65

4.2.3 Minimum feature resolution . . . . . . . . . . . . . 65

4.2.4 3D Structure Fabrication $\ldots \ldots$. . . . . . . . . . . . 67

4.2 .5 FTIR analysis . . . . . . . . . . . . . . 68

4.2.6 Cross-sectional morphology and composition analysis . . . . 69

4.2.7 Electrochemical activity . . . . . . . . . . . . . . 72

4.2 .8 Electrical conductivity . . . . . . . . . . . . . . . 72

4.2.9 Conclusions on pyrrole-UDMA hybrid polymer formulation . . . 75

4.3 Photopolymerization of pyrrole-BEMA hybrid polymer formulation . . . 77

4.3.1 Design of pyrrole-BEMA hybrid polymer formulation . . . . . . 77

4.3.2 Depth of cure . . . . . . . . . . . . . . . . . . . . . . 79

4.3 .3 Electrochemical activity . . . . . . . . . . . . . . . 79

4.3 .4 Electrical conductivity . . . . . . . . . . . . 80

4.3.5 3D structure fabrication $\ldots \ldots \ldots \ldots$. . . . . . . . . 81

4.3.6 Multi-material structure fabrication . . . . . . . . . . 83

4.3.7 Conclusions on pyrrole-BEMA photopolymer formulation . . . . 85

4.4 Photopolymerization of pyrrole-silver in commercial photopolymer formu-

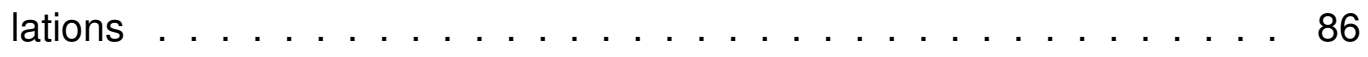

4.4.1 Integration commercial photopolymer formulations . . . . . 86

4.4.2 Electrical conductivity . . . . . . . . . . . . . . 87

4.4.3 Conclusions on commercial formulations . . . . . . . . 87

4.5 Chapter summary . . . . . . . . . . . . . . . . . . . . 88

List of references . . . . . . . . . . . . . . . . . 89 
5 Application of 3D conjugated polymer transducers

5.1 Actuation performance of pyrrole-BEMA hybrid polymer formulation . . . 93

5.1 .1 Methods . . . . . . . . . . . . . . . 93

5.1 .2 Results and discussion $\ldots \ldots \ldots$. . . . . . . . . . 93

5.2 Strain sensing performance of pyrrole-BEMA hybrid polymer formulation 96

5.2 .1 Methods . . . . . . . . . . . . . . . . 9 97

5.2 .2 Cyclic strain response . . . . . . . . . . . . . . . . . 98

5.2 .3 Strain to break response . . . . . . . . . . . . . . . . 999

5.2 .4 Discussion . . . . . . . . . . . . . . . . . . . . 101

5.3 Chapter summary . . . . . . . . . . . . . . . . . . . . . 102

List of references . . . . . . . . . . . . . . . . . . . 103

6 Concluding Remarks $\quad 104$

6.1 Summary of conclusions . . . . . . . . . . . . . . 104

6.2 Summary of contributions . . . . . . . . . . . . . 105

6.3 Recommendations for future research $\ldots \ldots \ldots \ldots$

$\begin{array}{ll}\text { Appendices } & 108\end{array}$

$\begin{array}{ll}\text { A Prototype fixed-surface vat polymerization design } & 109\end{array}$

B Prototype free-surface vat polymerization design 112

$\begin{array}{ll}\text { C Curriculum vitæ } & 115\end{array}$ 


\section{List of Tables}

3.1 Definition of variables in Equation $3.1 \ldots \ldots \ldots \ldots$

3.2 Definition of variables in Equation $3.2 \ldots \ldots \ldots \ldots$

4.1 Elemental composition of the hybrid photopolymer $\ldots \ldots \ldots$. . . 72 


\section{List of Figures}

2.1 Vat polymerization techniques ............... 8

2.2 Projection vat polymerization methods . . . . . . . . . . . . . . . . . 9

2.3 Slide peel release mechanism . . . . . . . . . . . . . . . . . . . . . 11

2.4 Flex vat release mechanism f . . . . . . . . . . . . . . . . . . . 11

2.5 Continuous liquid interface production additive manufacturing technique 12

2.6 Redox state of polypyrrole . . . . . . . . . . . . . . . . . . . . . 19

2.7 Trilayer conjugated polymer actuator design . . . . . . . . . . . . . 20

2.8 The oxidative polymerization reaction for polypyrrole . . . . . . . . . 22

3.1 Fixed-surface modifications made to the B9Creator 3D printer . . . . . . 33

3.2 Free-surface modifications made to the B9Creator 3D printer . . . . . . 34

3.3 CAD model of the DLP light engine assembly . . . . . . . . . . . . . . 38

3.4 Schematic representation of the lamination procedure . . . . . . . . . . 40

3.5 CAD model of the motorized laminating system . . . . . . . . . . . . . 41

3.6 CAD model of the multi-material system . . . . . . . . . . . . . . 43

3.7 CAD model of the build plate system . . . . . . . . . . . . . . . . . 44

3.8 Schematic diagram of the shielding gas system . . . . . . . . . . . . 45

3.9 Schematic diagram of the control system . . . . . . . . . . . . . . 46

3.10 CAD model of the system enclosure . . . . . . . . . . . . . . . . . 49

3.11 CCD sensor made from a disassembled Logitech C170 webcam . . . . 52 
3.12 Sample CCD images from the light engine focal length algorithm . . . 53

3.13 Failed multi-pillar fabrication job . . . . . . . . . . . . . . 54

3.14 Height of the liquid photopolymer over time $\ldots \ldots \ldots \ldots$

3.15 3D printed $6 \mathrm{~mm}$ tall chess rook $\ldots \ldots \ldots \ldots \ldots$

$3.163 \mathrm{D}$ printed $2.5 \mathrm{~mm}$ tall standard benchmarking model made from a commercial photopolymer . . . . . . . . . . . . . 56

4.1 Emission spectrum of the projector and absorption spectrum of the $\mathrm{H}-\mathrm{Nu}$ 470 photoinitiator . . . . . . . . . . . . . . . 59

4.2 Time-resolved UV-VIS spectroscopy of the pyrrole-silver formulation . . . 60

4.3 Photopolymerized pyrrole-silver formulation . . . . . . . . . . 60

4.4 Failed structure made from the pyrrole-silver formulation with the fixedsurface technique . . . . . . . . . . . . . . . 61

4.5 Failed structure made from the pyrrole-silver formulation with the freesurface technique . . . . . . . . . . . . . . . 62

4.6 Depth of cure results for the pyrrole-UDMA polymer formulation. . . . . 65

4.7 Results from the feature resolution experiment $\ldots \ldots \ldots 66$

4.8 Structures made from pyrrole-UDMA formulation . . . . . . . . 67

4.9 FTIR spectra of photopolymerized PPy, UDMA, and a hybrid pyrroleUDMA formulation . . . . . . . . . . . . . . . 69

4.10 SEM of the cross-section of a hybrid pyrrole-UDMA photopolymer structure 70

4.11 EDAX elemental mapping of the pyrrole-UDMA formulation $\ldots \ldots 71$

4.12 Cyclic voltammetry of the hybrid pyrrole-UDMA formulation . . . . . . 73

4.134 -point probe apparatus . . . . . . . . . . . . . . . . . 74

4.14 Electrical conductivity of the pyrrole-UDMA hybrid polymer . . . . . . 76

4.15 Emission spectrum of the light engine and absorption spectrum of the TPO photoinitiator and Tinuvin 477 UV absorber $\ldots \ldots \ldots \ldots$ 
4.16 Bridge structure used to characterize the depth of cure of the pyrroleBEMA formulation . . . . . . . . . . . . . . . . 79

4.17 Depth of cure results for the pyrrole-BEMA polymer formulation confirms the hybrid polymer formulation agrees well with the depth of cure model described in Section 2.2.5 . . . . . . . . . . . . . . . . . 80

4.18 Cyclic voltammogram of the hybrid pyrrole-BEMA polymer formulation . 81

4.19 Electrical conductivity of the pyrrole-BEMA hybrid polymer . . . . . . . 82

4.20 3D printed $2.5 \mathrm{~mm}$ standard benchmarking model made from a pyrroleBEMA hybrid polymer formulation $\ldots \ldots \ldots$. . . . . . 82

4.21 Multi-material 3D printed prototype microfluidic channel . . . . . . . 84

4.22 Electrical conductivity of the hybrid polymer of pyrrole and a conventional photopolymer . . . . . . . . . . . . . . . . 88

5.1 Electrochemical stimulation apparatus for measuring the actuation response of Pyrrole-BEMA hybrid polymer samples . . . . . . . . . . . . 94

5.2 Electrochemical excitation voltage signal and resulting current flow $\ldots 95$

5.3 The tip displacement of an actuated polymer film in response to the excitation voltage signal . . . . . . . . . . . . . . . . 96

5.4 Pyrrole-UDMA hybrid polymer sample installed in a CellScale UniVert materials testing instrument $\ldots \ldots \ldots \ldots$. . . . . . . 98

5.5 The typical response of the hybrid pyrrole-UDMA polymer sample under cyclic tensile loading $\ldots \ldots \ldots \ldots$

5.6 Normalized resistance of the hybrid polymer sample in the stretched and relaxed states over 200 loading cycles. . . . . . . . . . . . . 100

5.7 The gauge factor of the hybrid polymer sample over 200 loading cycles shows a linear decrease in the gauge factor with increased cycling. . . 100

5.8 Typical strain to break response for hybrid polymer samples with a plot of normalized resistance versus strain. . . . . . . . . . . . . 101 
A.1 Unmodified B9Creator 3D printer used in the preliminary study of vat polymerization of conjugated polymers. . . . . . . . . . . . . . . . . 110

A.2 Modifications made to the B9Creator 3D printer to reduce the build volume of the polymer vat. . . . . . . . . . . . . . . . . 110

B.1 Modifications made to the B9Creator 3D printer to print with the freesurface vat polymerization technique. . . . . . . . . . . . . 113

B.2 Schematic diagram of the prototype free-surface additive manufacturing system . . . . . . . . . . . . . . . . . . . . . . . 114 


\title{
List of Acronyms and Symbols
}

\author{
Acronyms
}

BEMA bisphenol A ethoxylate dimethacrylate

CAD computer aided design

CCD charge-coupled device

CLIP continuous liquid polymer interface

CNT carbon nanotubes

$\mathrm{CP} \quad$ conjugated polymer

CV cyclic voltammetry

DLP digital light processing

EDAX Energy dispersive $\mathrm{X}$-ray spectroscopy

FEP Fluorinated ethylene propylene

FTIR Fourier-transform infrared spectroscopy

LCD liquid crystal display

LiTFSI lithium bistrifluoromethanesulfonimidate

MEMS microelectromechanical systems

PDMS polydimethylsiloxane

PEGMA poly(ethylene glycol) methyl ether methacrylate

SEM scanning electron microscopy

SLA sterolithography

TPO 2,4,6-trimethylbenzoyl-diphenyl-phosphineoxide 


$$
\begin{array}{ll}
\text { UDMA } & \text { urethane dimethylacrylate } \\
\text { UV } & \text { ultraviolet }
\end{array}
$$

\section{Greek symbols}

$\begin{array}{ll}\rho & \text { resistivity } \\ \sigma & \text { apparent conductivity } \\ \nu & \text { Poisson's ratio }\end{array}$

\section{Latin symbols}

$C_{B P} \quad$ Amount of resin displacement per depth of the build plate elevator into the polymer vat

$C_{d}$ cured photopolymer depth

$d$

probe spacing

Position of the build plate when measuring the build plate position in step 1 of the liquid level calibration

$\Delta l \quad$ change in length

$D_{p} \quad$ penetration of light into the photopolymer

$\Delta R \quad$ change in resistance

$d_{R S} \quad$ Position of the build plate when measuring the liquid photopolymer surface

E irradiated light energy

$E_{c} \quad$ critical light exposure to initiate polymerization

GF gauge factor

I current

lo initial length

$R_{0} \quad$ initial resistance

V voltage

$x_{B P} \quad$ Vertical laser sensor stage position plus the laser sensor reading when measuring the build plate position in step 1 of the liquid level calibration 


\begin{tabular}{|c|c|}
\hline$x_{C C D}$ & $\begin{array}{l}\text { Vertical laser sensor stage position plus the laser sensor reading when } \\
\text { measuring the CCD sensor position in step } 2 \text { of the focal length calibra- } \\
\text { tion }\end{array}$ \\
\hline$x_{f}$ & $\begin{array}{l}\text { Position of the projector stage when the image is in focus on the CCD } \\
\text { sensor }\end{array}$ \\
\hline$x_{p}$ & Projector stage position \\
\hline$x_{R S}$ & $\begin{array}{l}\text { Vertical laser sensor stage position plus the laser sensor reading when } \\
\text { measuring the liquid photopolymer surface in step } 3 \text { of the liquid level } \\
\text { calibration }\end{array}$ \\
\hline$x_{c}$ & Starting build plate position for vat polymerization \\
\hline
\end{tabular}




\section{Chapter 1}

\section{Introduction}

Smart materials possess unique properties that allow them the convert energy from one form to another through intrinsic material properties. Conjugated polymers are a class of smart materials that are electrically and ionically conductive due to a conjugated polymer backbone consisting of alternating single and double bonds. The ionic conductivity property of conjugated polymers has numerous applications within advanced sensing and actuation technologies. Conjugated polymers can be used in mechanical actuation technologies due to ion driven volumetric expansion and contraction along with sensing technologies which utilizes a change in their electrical conductivity in response to external stimuli.

The intractable nature of conjugated polymers has prevented this material from being used with traditional polymer forming techniques such as injection molding. Conjugated polymers are typically synthesized directly into their final shape via in situ polymerization techniques such as chemical, electrochemical and photopolymerization. Electropolymerization is commonly used to form thin polymer film actuators due to the desirable mechanical and electrical properties of the produced polymer film. Little work has been performed on the photopolymerization technique since it possesses a low yield and produces a conjugated polymer with a lower conductivity compared to other 
polymerization techniques.

Thin conjugated polymers films produced via electropolymerization synthesis method have been used to make actuators capable of $35 \%$ volumetric expansion and contraction under a low excitation voltage of $\pm 1 \mathrm{~V}$ (Spinks et al., 2009). Thin film actuators are however constrained to simple linear and bending motion due to the monolithic nature of the device. This limitation illustrates a need for the development of a new fabrication technique that is capable of forming 3D conjugated polymer structures to produce more complex modes of actuation.

\subsection{Objectives}

This research project aims to develop a photosensitive conjugated polymer formulation and light-based additive manufacturing system to create 3D active conjugated polymer structures with microscale features. This goal is described with the following research objectives:

- Investigate the capability of light based additive manufacturing to produce 3D conjugated polymer structures. The first objective is to perform an initial investigation on the use of photosensitive conjugated polymer formulations with light based additive manufacturing systems to realize 3D conjugated polymer structures. This investigation will shed light on the primary challenges of this technique and will provide insight into the development of an optimal fabrication system and photosensitive conjugated polymer formulation.

- Develop a light based additive manufacturing system for use with photosensitive conjugated polymer formulations. The second objective will use the lessons learned from the first objective to develop a fabrication system with features to aid in the fabrication of 3D conjugated polymer structures. The additive manufacturing system will be designed with the goal of fabricating microscale structures 
to produce devices for applications within the fields of microelectromechanical systems (MEMS) and microfluidics.

- Develop a photosensitive conjugated polymer formulation for use with light based additive manufacturing. The third objective will improve upon existing photosensitive conjugated polymer formulations to make them compatible with the light based additive manufacturing system developed within the second objective. Additional additives will be investigated to realize improved feature resolutions and conjugated polymer transduction properties.

- Investigate the transduction properties of photopolymerized conjugated polymer structures. The fourth objective will use the outcome from the second and third objectives to investigate the actuation and mechanical strain sensing performance of the developed photosensitive conjugated polymer formulation. The completion of this objective will provide insight into the types of devices that can be produced with the developed light based additive manufacturing system and photosensitive conjugated polymer formulation.

\subsection{Major contributions}

This thesis conveys the following major contributions to the scientific body of knowledge:

- The design of a multi-material free-surface vat polymerization additive manufacturing system. A novel multi-material system is developed for the fabrication of 3D conjugated polymers devices. The fabrication system employs a DLP light engine capable of selectively curing features with a resolution of $5 \mu \mathrm{m}$. A laminating system allows the apparatus to create a target layer thickness of $5 \mu \mathrm{m}$ to build 3D structures. The multi-material feature enabled the production of devices with 
both active and passive structures.

- A hybrid polypyrrole formulation that is compatible with the vat polymerization additive manufacturing technique. A photosensitive conjugated polymer formulation is presented that was designed for use with the vat polymerization additive manufacturing system for the production of 3D conjugated polymer structures. The polymer formulation consists of a blend of the conjugated polymer polypyrrole and traditional photopolymers to improve the mechanical properties of the overall polymer while retaining the smart material properties of the conjugated polymer.

- Strain sensing and actuation characterization of hybrid polypyrrole structures. The developed hybrid polypyrrole formulation was found to have piezoresistive and electromechanical transduction properties which may be utilized for mechanical strain sensing and actuation applications. Strain to break experiments found that the hybrid polypyrrole formulation was compliant with a maximum yield strain of $13 \%$. Further studies are needed to shed light on the complex long term piezoresistive response. A small amount of volumetric expansion and contraction was observed when the hybrid polymer was electrochemically stimulated in a LiTFSI solution. This preliminary study may be used to inform the design of devices featuring hybrid polypyrrole structures.

\subsection{Organization of the thesis}

Chapter 2, Background and state of the art provides background information and a review of the state of the art to orient the reader through the subsequent chapters. A review of additive manufacturing technology is provided with an emphasis on lightbased technologies. A review of the state of the art in the fields of vat polymerization and photosensitive conjugated polymer formulations is presented. Chapter 3, Fabrica- 
tion system development provides a detailed description of a custom vat polymerization additive manufacturing system which is used for the fabrication of 3D conjugated polymer structures. Chapter 4, Development of a photosensitive conjugated polymer formulation presents a photosensitive conjugated polymer formulation which has been optimized for the fabrication of micro-scale 3D conjugated polymer structures using the developed vat polymerization additive manufacturing system. Chapter 5, Application of $3 D$ conjugated polymer transducers investigates the application of the developed photosensitive conjugated polymer formulation to sensing and actuation technologies. Electrochemical stimulation is used to study the electroactive response of the conjugated polymer formulation. The piezoresistive properties of the conjugated polymer are studied with strain to break and cyclic loading experiments. Lastly, Chapter 6, Concluding Remarks summarizes the conclusions and contributions from this thesis. Recommendations for future work on the fabrication of 3D conjugated polymer structures using the vat polymerization additive manufacturing technique are discussed.

\section{List of references}

Spinks, G. M., Alici, G., McGovern, S., Xi, B. and Wallace, G. G. (2009), Conjugated Polymer Actuators: Fundamentals, in 'Biomedical Applications of Electroactive Polymer Actuators', John Wiley \& Sons, Ltd., West Sussex, United Kingdom, pp. 193227. 


\section{Chapter 2}

\section{Background and state of the art}

This chapter will summarize the relevant background information on vat polymerization additive manufacturing technology and conjugated polymers from which this thesis is based upon. Various vat polymerization additive manufacturing techniques will be presented along with the advantages and disadvantages of each. The components of photosensitive polymer formulations used with vat polymerization are reviewed. Finally, conjugated polymers are discussed with a focus on photosensitive formulations.

\subsection{Review of vat polymerization additive manufactur- ing technology}

The rapid advancement of additive manufacturing technologies has inspired the development of a wide range of new techniques. Additive manufacturing technologies can be divided into the following groups: material extrusion, powder bed fusion, binder jetting, sheet lamination, material jetting and vat photopolymerization (ASTM, 2015). Despite the differences in their operating principles, they all utilize a layer-by-layer fabrication method to create 3D objects. Extensive research has been put forth to advance the resolution of additive manufacturing technologies to the micro and nano-scale for its 
potential applications in MEMS, lab-on-a-chip and energy storage (Mao et al., 2017).

The vat polymerization technique is commonly used in commercial and research additive manufacturing systems to create high resolution 3D structures. This technique uses either a laser or projection system to selectively cure 2D layers of a photosensitive polymer resin to build 3D objects layer-by-layer. The stereolithography (SLA) technique, illustrated in Figure 2.1a uses a 2D scanning laser to cure the photopolymer.

Projection based systems, illustrated in Figure 2.1b, cure the full 2D layer at once by projecting a black and white image onto the $2 \mathrm{D}$ curing plane which results in reduced complexity, positioning errors, and build times compared to the SLA technique (Mueller, 2012). The projected image can be generated using digital light processing (DLP) light engine which consists of a micromirror array to reflect the light of each pixel toward or away from the output to display the desired image. Liquid crystal display (LCD) technology can be used as a dynamic mask to generate the projected imaged as well. The advancement of LCD technology in consumer devices has resulted in the highresolution displays which can operate in the UV spectrum, allowing them to be used for vat polymerization additive manufacturing. The advantage of DLP projection systems over LCD displays is the superior black level which prevents photopolymerization outside the desired areas (Sun et al., 2005).

An emerging vat polymerization technique for the fabrication of micro-nano 3D structures is two-photon polymerization. This technique, shown schematically in Figure 2.1c, photopolymerizes polymer at the intersection of two lasers. 3D structures can be produced by scanning the point of intersection within a vat of polymer. This method is unique compared to laser and projection vat polymerization approaches as it doesn't require a recoating step which leads to faster fabrication times and reduced complexity. Spatial resolutions of $80 \mathrm{~nm}$ have been achieved with the two-photon polymerization method by utilizing high precision lasers and positioning systems (Mao et al., 2017). The two-photon approach is attractive for the fields of microphotonics, microfluidics, and 


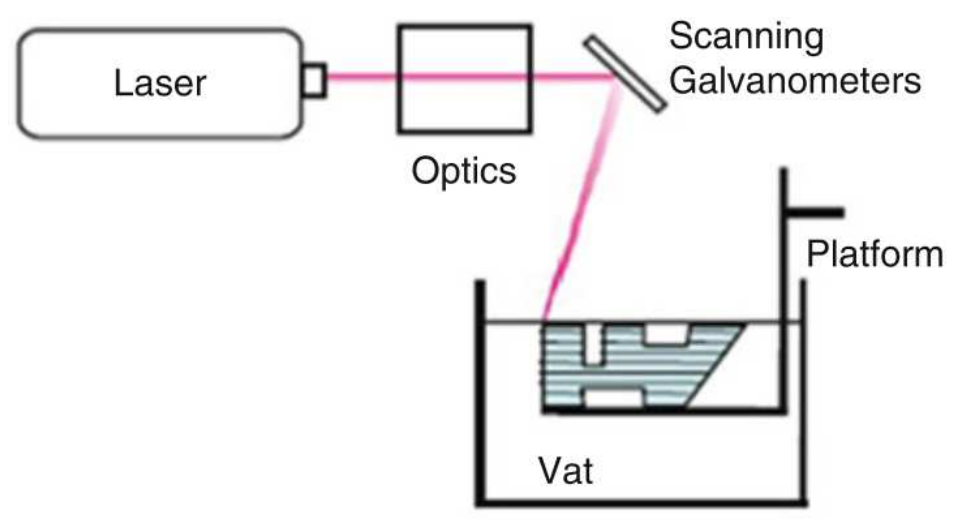

(a)

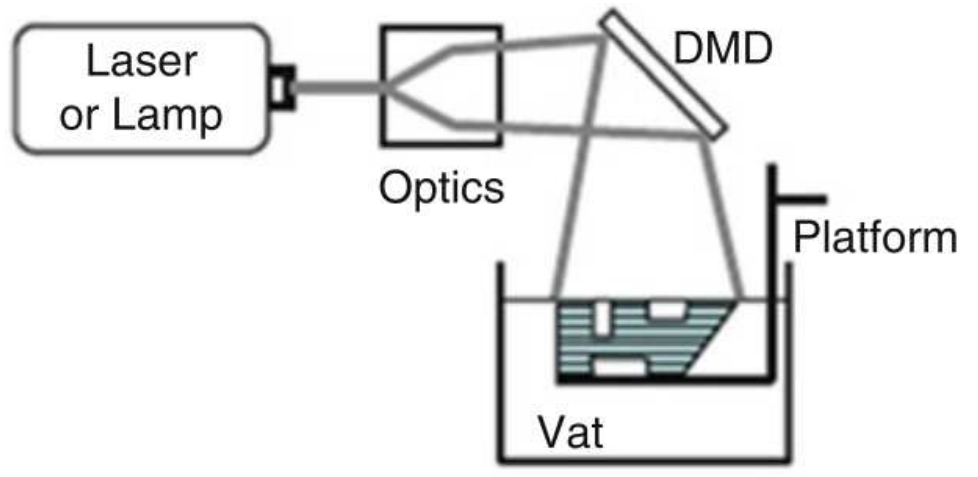

(b)

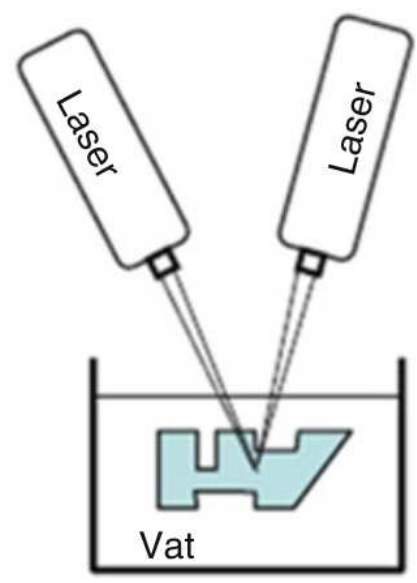

(c)

Figure 2.1: Schematic representation of (a) laser, (b) projection, and (c) two-photon vat polymerization technique (@ Mueller, 2012, included with permission)

biomedical devices but this technique has the disadvantages of being costly, limited to small build volumes and is restricted to photopolymer that have a high transparency to the laser beams.

Projection vat polymerization additive manufacturing techniques use either a fixedsurface or free-surface fabrication method. For the fixed-surface method, seen in Figure 2.2a, thin layers of the photopolymer formulation are selectively cured between a window in the bottom of a resin vat and the previously cured layer to form the 3D object upside down. The free-surface method, seen in Figure 2.2b, creates the 3D object right side up by selectively curing polymer layers on top of the previous layer. 


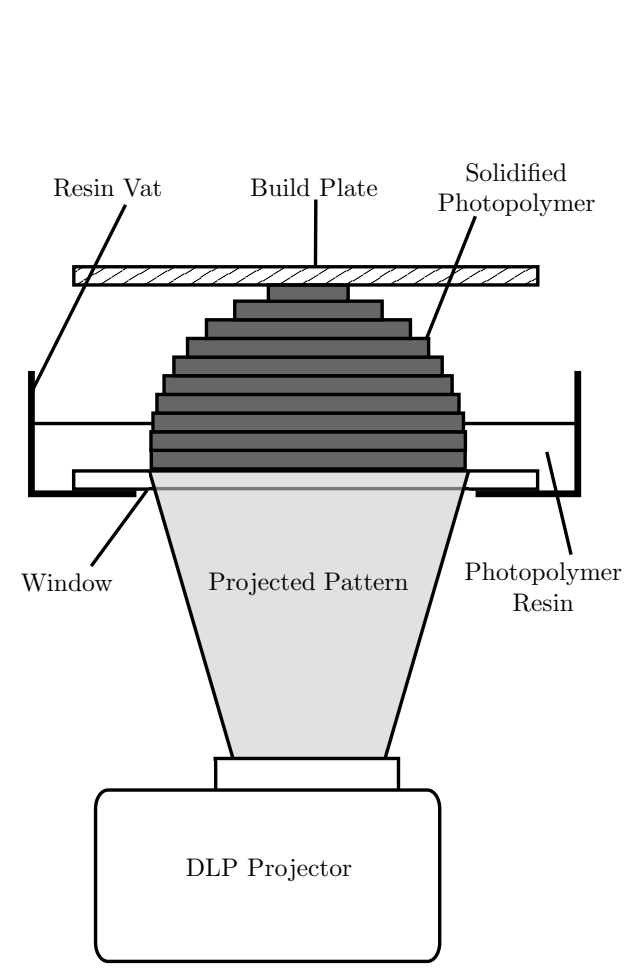

(a)

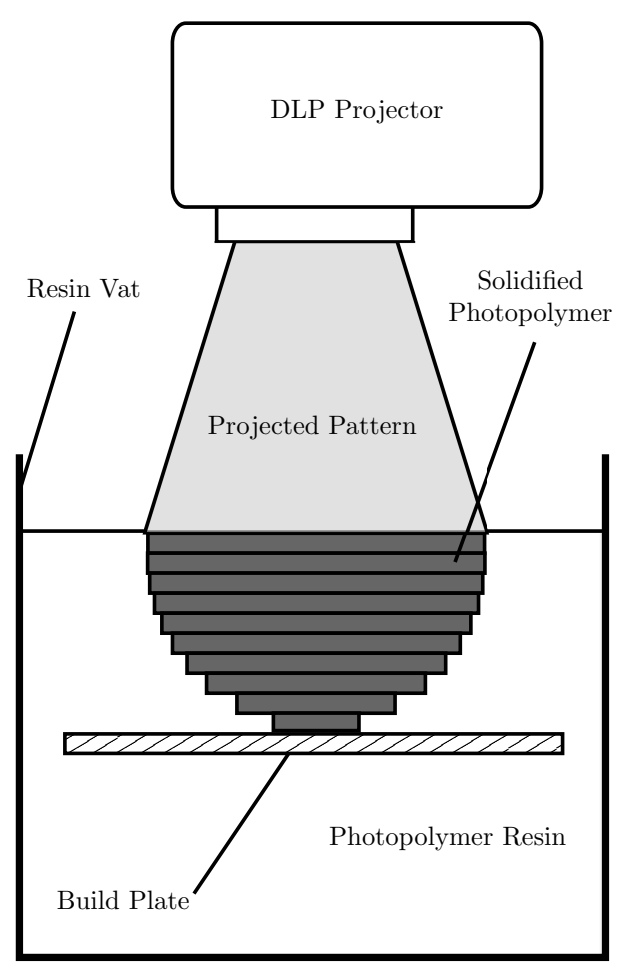

(b)

Figure 2.2: Schematic diagram of the (a) fixed-surface and (b) free-surface DLP fabrication methods (@ Cullen and Price, 2018, included with permission) 
The fixed-surface method often requires a non-stick coating on the window to reduce adhesion between the cured polymer layer and the window. This technique is incompatible with low stiffness materials because the layers will tear apart during the peeling process. The fixed-surface technique is preferred for low cost commercial systems since it provides precise control of the cured layer thickness with the use of a linear stage. Various techniques have been investigated to reduce the peeling force between the newly cured polymer layer and the window, the most popular of which is to use an oxygen permeable material on the window to create an uncured dead zone above the window. Oxygen dissolved in a liquid photopolymer resin will react with radicals generated from the photoinitiator, drastically inhibiting polymerization. Transparent and oxygen permeable polymers such as polydimethylsiloxane (PDMS) are commonly used as an oxygen permeable film on the window. The film is usually aerated every layer by squeegeeing the liquid photopolymer to replenish oxygen within the film. The design of the vat can aid in reducing the peeling forces on the part. Instead of pulling the cured layer perpendicular to the window, the layer can be slid sideways from the build window, as illustrated in Figure 2.3 (Joyce, 2017). A flex vat design, shown in Figure 2.4 , is another common solution which allows the layer to be peeled at an angle (Sayao, 2016). Recent advances in fixed-surface technique have eliminated the need for the peeling step. The continuous liquid interface production (CLIP) technique, illustrated in Figure 2.5, uses a thin transparent Teflon membrane with a high oxygen permeability as the window to create an uncured dead zone that is constantly replenished with oxygen since the bottom of the film is exposed to air (Tumbleston et al., 2015). The CLIP technique eliminates the need to peel each layer from the window after curing. The build platform is raised at a constant rate while the projected 2D image continuously changes according to the geometry being produced. This technique results in a greatly decreased fabrication time.

The free-surface method eliminates the problem of peeling forces by curing the 


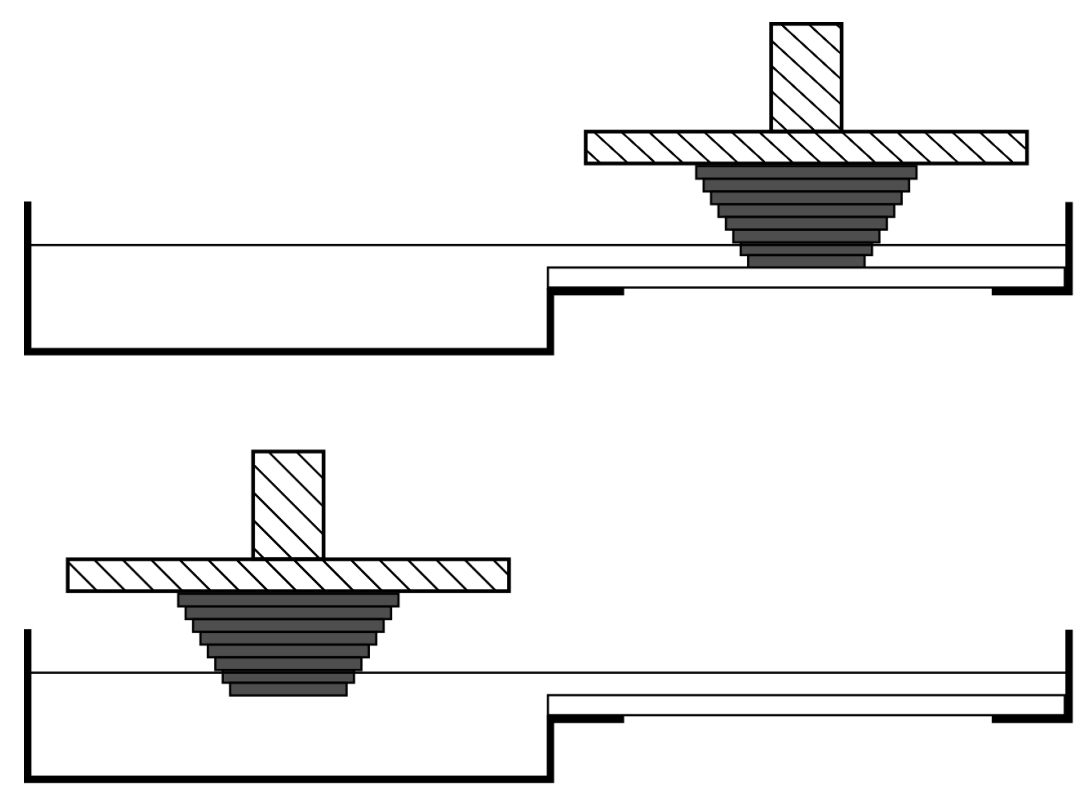

Figure 2.3: Schematic diagram of the slide peel release mechanism used within vat polymerization additive manufacturing systems.
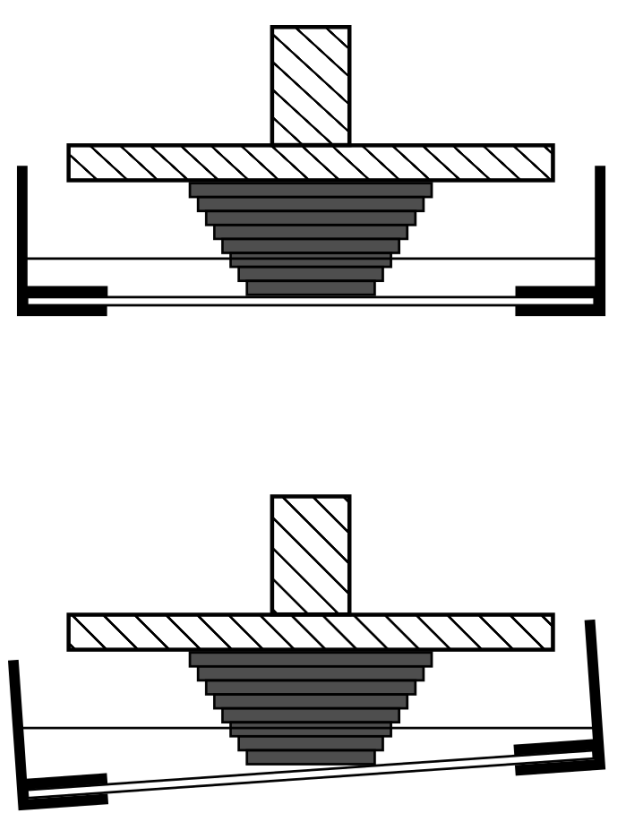

Figure 2.4: Schematic diagram of the flex vat release mechanism used within vat polymerization additive manufacturing systems. 


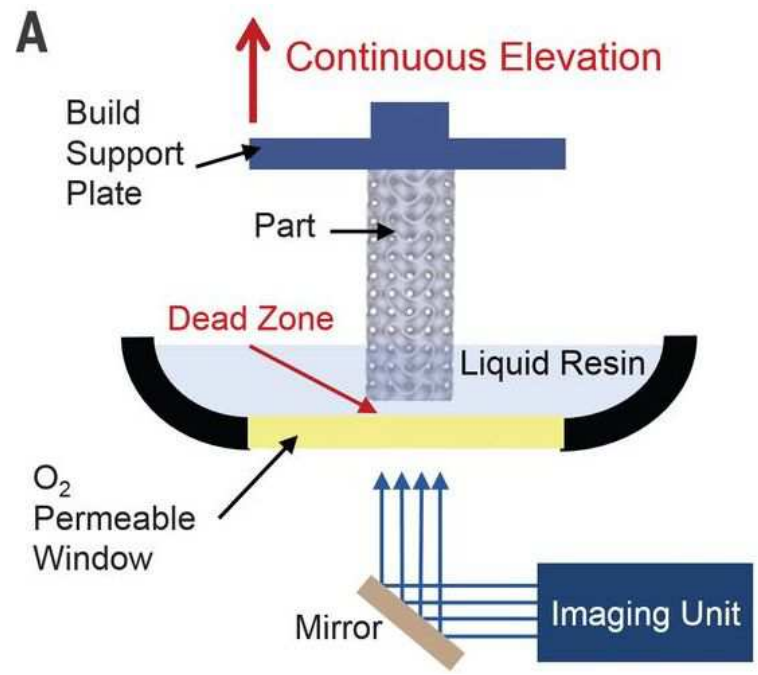

Figure 2.5: Schematic diagram of the continuous liquid interface production (CLIP) additive manufacturing technique (@) Tumbleston et al., 2015, included with permission).

polymer layer on top of the previously cured layer. This method however requires more advanced techniques to control the cured layer thickness due to surface tension forces between the photopolymer resin and the previously cured layer. Laminating techniques are typically utilized to form thin polymer layers with a high precision (Renap and Kruth, 1995; Kengo Kobayashi and Koji Ikuta, 2007; Wang et al., 2009; Pham and Ji, 2003). Consideration must be given to oxygen inhibition with the free-surface technique since the curing layer is exposed to air. The curing surface is typically blanketed with an inert gas, or the polymer vat is in an inert atmosphere to compensate for this effect (Ligon et al., 2014).

While most hobbyist light-based 3D printers use the fixed surface technique due to its simplicity in layer thickness control, many high end commercial systems such as the 3D-Systems SLA 250 use the free surface technique since it is able to print a wide range of materials. The primary challenge with the free surface technique is the ability to laminate a thin and even polymer layer on top of the previously cured layer.

The simplest technique for laminating photopolymer layers with the free surface technique is the dip method. In this technique, the build plate is first lowered into a 
photopolymer vat until the liquid photopolymer flows over the previous layer, the build plate raised until the previously produced layer is one layer thickness below the surface of the liquid photopolymer. The produced layer thickness can be accurately controlled with: a linear stage attached to the build plate, syringe pump dispensing photopolymer into the resin vat, or volume displacement techniques (Wang et al., 2009; Hafkamp et al., 2018). The dip technique works well for low precision systems which utilize thick layer thicknesses or with low viscosity photopolymers since surface tension between the liquid photopolymer and the previously cured layer will prevent a thin photopolymer layer from being formed.

To laminate thin photopolymer layers for high resolution 3D printing, a wiper system is commonly used. The wiping procedure consists of: dipping the build plate into the photopolymer vat to flow photopolymer resin over the previously cured layer, using a mechanical wiper to remove excess photopolymer on top of the previously cured layer, positioning the previously cured layer is one layer thickness below the photopolymer surface in the vat, and finally allowing the laminated photopolymer layer to stabilize before the photopolymer layer is ready for curing.

The distance between the wiper and the previous layer must be accurately controlled to successfully complete the laminating procedure. If the produced layer is too thick, the cured layer won't properly adhere to the previous layer. Alternatively, if the layer is too thin, the wiper risks colliding with the previous layer. The velocity that the wiper passes over the surface should be carefully controlled. Renap and Kruth (1995) showed that laminating a layer over a part with a trapped volume can result in an uneven layer to be produced because the blade will remove too much liquid from the trapped volume. This effect can be compensated for by using a slower wiping velocity or increased settling delay, but this will inevitably make the production time much longer. Kengo Kobayashi and Koji Ikuta (2007) have shown that a layer thickness of $10 \mu \mathrm{m}$ can be produced with careful design of the wiper geometry. The angle of the wiper and 
the coating on the rear side of the wiper influence the height of meniscus on the wiper which influences the uniformity of the produced layer.

Fabricating multi-material structures with light-based processes is more challenging compared to material extrusion techniques, but several groups have found effective solutions due to the advantages of multi-material printing (Zhou et al., 2013; Choi et al., 2010; Holtrup, 2015). Most implementations of multi-material vat polymerization additive manufacturing systems have multiple polymer vats filled with different photopolymers and a cleaning station filled with a solvent to clean the solidified photopolymer when switching between photopolymer vats. The multi-material feature is valuable for multi-coloured parts, components with varying mechanical properties for applications such as hard cases with soft ergonomic grips, and components with embedded electrical connections by using a polymer with a conductive filler.

\subsection{Review of photopolymerization}

The photopolymer formulation used within the vat polymerization additive manufacturing technique must be carefully designed to meet the material property requirements for the target application while being compatible with the fabrication technique to create

the desired structures. This section will provide a broad review of the primary components in photopolymer formulations and techniques to characterize the properties of photosensitive polymer formulations.

Photopolymer formulations typically consist of monomers, oligomers, and a photoinitiating system which initiates chain growth polymerization to convert the monomers and oligomers into polymer molecules. 


\subsubsection{Photoinitiating system}

The photoinitiating system is used to generate reactive species when irradiated with light to initiate chain growth polymerization of the oligomers and monomers to form long polymer chains. The two most common classes of photoinitiators are radical and cationic.

Radical photoinitiators generate reactive radicals upon irradiation with light to initiate polymerization of acrylate-based polymers. Radical photoinitiators are highly reactive leading to fast cure times. The generation of radicals is subdivided into Type I and Type II photoinitiators. For Type I photoinitiators, the excited state of the photoinitiator molecule quickly splits in two, producing two reactive radicals. Type II photoinitiators use a less direct method of generating radicals. The excited state of the Type II photoinitiator extracts a hydrogen from a suitable hydrogen donor to produce two radicals, one with the photoinitiator molecule and another more reactive radical from the hydrogen donor (Jenkins, 2000; Liska, 2011).

The excited state of radical photoinitiators can be deactivated before their able to generate free radicals. Deactivation of the excited photoinitiator can occur from quenching reactions with monomers in the formulation and oxygen dissolved liquid photopolymer (Liska, 2011). Care must be taken to ensure compatibility of the photoinitiator system with the monomers in the photopolymer formulation.

Cationic photoinitiators are used to polymerize epoxy-based polymers. Cationic photoinitiators generate a protonic acid (Bronsted or Lewis acid) when irradiated with light to initiate a ring opening reaction with epoxy monomers. The cationic polymerization reaction is slower compared to radical photoinitiation but it has other beneficial properties. The reactive species generated by the cationic polymerization chemistry are insensitive to oxygen allowing it to be used in a wider range of applications. The reactive cations are stable for a longer period of time resulting in a "dark cure" after the initiating light has been turned off which results in a higher degree of curing (Jenkins, 
2000; Liska, 2011).

The specific photoinitiating system used within a polymer formulation is selected based on the following criteria:

- Strong overlap between the absorbance spectrum of the photoinitiator and the emission spectrum of the light source

- Capable of generating reactive species to initiate polymerization of the monomers and oligomers in the polymer formulation

- High polymerization efficiency for the monomers and oligomers in the polymer formulation

\subsubsection{Oligomers}

A large portion of a photosensitive polymer formulations consists of oligomers. Oligomers are used compared to monomers for the bulk portion of a photopolymer formulation because they lead to longer chain lengths upon curing. Oligomers are usually classified based on their polymerization mechanism. Acrylate-based oligomers are initiated with hydrogen donor reaction from a radical photoinitiator while epoxy-based oligomers are initiated by a ring opening reaction from a cationic photoinitiator. Acrylate-based oligomers can be rapidly polymerized with short irradiation times which is favourable for decreased fabrication times. The radical polymerization reaction however can experience significant shrinkage of $5-20 \%$ leading to significant inaccuracies and curling of the polymer structures. Epoxy-based oligomers require longer irradiation times compared to acrylate oligomers, but experience less shrinkage upon curing, around 1-2\% (Mueller, 2012). The dark cure property of cationic photoinitiators leads to a stiff, but brittle material compared to acrylate polymers. Commercial polymer formulations incorporate both epoxy and acrylate oligomers to gain the benefits of each type of polymer. 


\subsubsection{Monomers}

Oligomers tend to have a high viscosity which can make laminating thin polymer layers challenging. Lower viscosity monomers are typically added to the polymer formulation to control the overall viscosity while still contributing to the polymerization reaction. This additive is typically referred to as a reactive diluent.

\subsubsection{Light absorber}

One of the most challenging features to fabricate with light-based 3D printing technology is overhangs. If the irradiated light can pass deep within the photopolymer, the layer thickness will be cured much thicker then desired. A common technique to combat this effect is to introduce a non-reactive component that absorbs light within a similar spectrum as the photoinitiator. This component will constrain light penetration to the desired layer thickness, preventing over curing when fabricating overhangs.

\subsubsection{Characterization of photopolymer formulations}

The reactivity of photopolymer formulations are characterized to identify the amount of irradiation required to cure a desired layer thickness. Photosensitive polymer formulations are typically characterized by a depth of cure experiment. In this experiment, the depth of polymerization is measured for a range of irradiation times.

The data obtained from this experiment is used to calculate a working curve equation which models the depth of cure with respect to the amount of irradiated light energy (Jacobs, 1992; Sun et al., 2005; Beluze et al., 1999) and is given by:

$$
C_{d}=D_{p} \ln \frac{E}{E_{c}},
$$

where $C_{d}$ is the cured depth, $D_{p}$ is the light penetration into the photopolymer, $E$ is the 
irradiated light energy and $E_{c}$ is the critical light exposure to initiate polymerization. This expression equates to a linear line on a semilogarithmic plot of irradiated light energy versus cured depth where $D_{\mathrm{p}}$ is the slope and $E_{\mathrm{c}}$ is the exposure energy axis intercept.

Another method to analyze the reactivity of a polymer formulation is real time Fourier transform infrared spectroscopy. In this technique, the infrared absorption spectrum of the polymer is monitored throughout the photopolymerization reaction. Location and intensity of peaks in the absorption spectrum of the polymer will shift as new chemical bonds are formed. This technique is valuable for hybrid polymer formulations where it is desired to match the cure time of polymers in the formulation (Kasisomayajula et al., 2016).

\subsection{Review of conjugated polymers}

An emerging area of research in the field of additive manufacturing is the development of $4 \mathrm{D}$ printing. In this field, the 3D printed materials can actuate in response to an external stimulus (Shin et al., 2017). A new potential material for 4D printing technology is conjugated polymers. This type of polymer has characteristic alternating single and double bonds along the polymer chain, also referred to as conjugation. The conjugation property makes this polymer electroactive which allows various properties of the polymer to be varied electrochemically. The conjugated polymer polypyrrole (PPy) is shown in its oxidized and reduced state in Scheme 2.6. PPy is a neutral polymer within the reduced state. When the polymer is oxidized, bond reorientation results in positive charges to form along the polymer chain. Anions diffuse into the polymer to neutralize the overall charge of the material. Within the reduced state, electrons held within the $\mathrm{Pi}$ bonds present at the double bond locations are not held as tightly held as with conventional polymers which allows this material conduct electricity. The electrical conductivity of the polymer is increased 10 orders of magnitude when switched to the oxidized state 

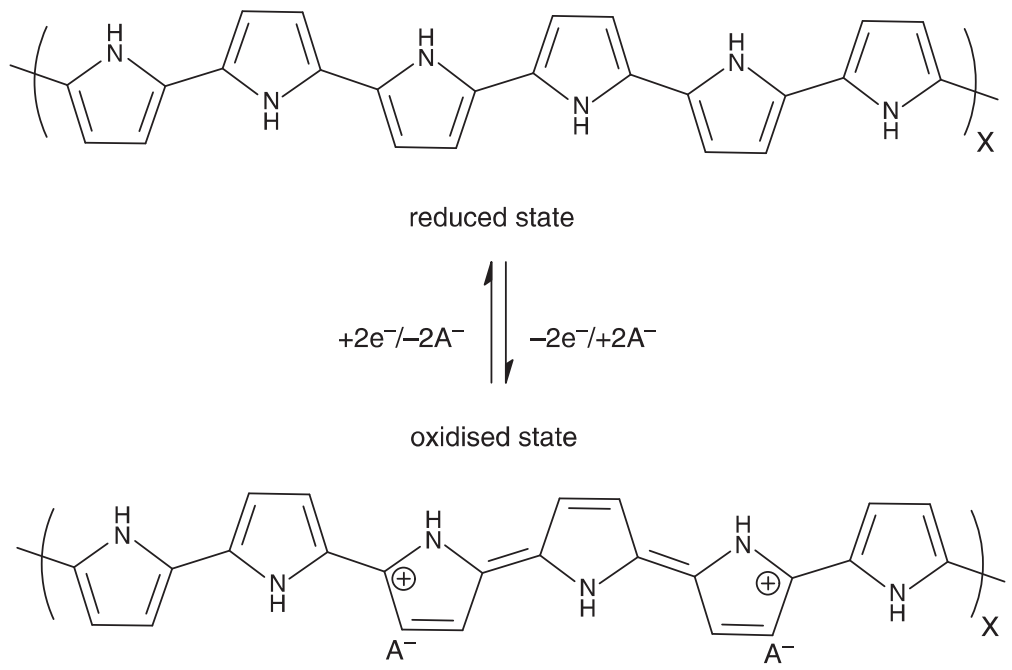

Figure 2.6: The oxidized and reduced states of the conjugated polymer polypyrrole (@) Wallace et al., 2009, included with permission)

since the charges developed along the polymer chain further increase the mobility of electrons along the polymer chain (Spinks, Alici, McGovern, Xi and Wallace, 2009). The intrinsic conductivity property of conjugated polymers has found applications with organic electronics, sensing and electrostatic dissipation (Wallace et al., 2009).

The diffusion of ions into the polymer to neutralize the overall charge of the material has been exploited to create actuators with conjugated polymers. The ingress and egress of ions when the polymer is switched between the oxidized and reduced states causes volumetric expansion and contraction which can be harnessed to perform mechanical work. Studies have shown that the amount of volumetric expansion and contraction can be maximized by electrochemical switching the polymer in an electrolyte solution containing cations and anions that have a relatively large size difference. For instance, when polypyrrole is operated in a solution of LiTFSI, the polymer experiences a volumetric expansion of $35 \%$ in the oxidized state (Spinks, Alici, McGovern, Xi and Wallace, 2009).

Actuation of conjugated polymers is performed in an electrochemical cell containing the desired electrolyte solution. Due to the electrochemical nature of this actuator, 


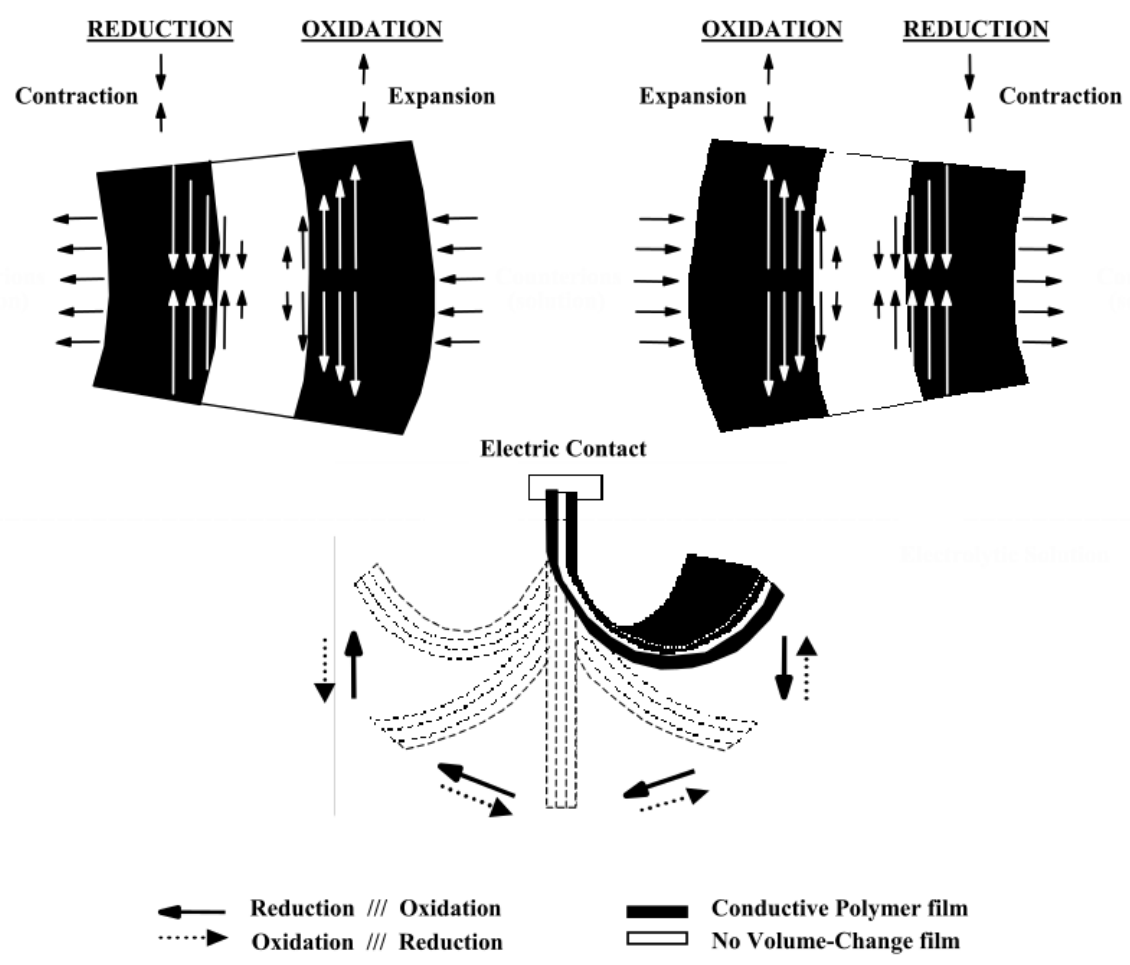

Figure 2.7: Trilayer conjugated polymer actuator consisting of a porous membrane containing the desired electrolyte with conjugated polymer coated on both sides (C) Smela, 2003, included with permission)

low voltages of $\pm 1 \mathrm{~V}$ are required to operate this material. The choice of cations and anions in the solution, solvent for the electrolyte, and the preparation conditions of the polymer impact the performance of conjugated polymer actuators. To overcome the limitation of actuating conjugated polymers in an electrolyte solution researchers commonly utilize a trilayer actuator design shown in Figure 2.7. In this configuration, a porous membrane containing the desired electrolyte is coated with the conjugated polymer on both sides. When an electric potential is applied across the two polymer layers, ions will diffuse from one polymer layer to the other. When the potential is reversed, ions will diffuse to the other electrode. The ion driven expansion of one polymer coating and the contraction of the other will cause the device to bend in the direction of the contracted coating. This actuator configuration can be sealed, allowing the polymer to be operated outside of solution. 
PPy is commonly studied for sensing and actuation applications due to its large actuation strains and ease of synthesis (Spinks, Alici, Mcgovern and Xi, 2009). The simplified polymerization reaction for PPy is shown in Scheme 2.8. The polymerization of pyrrole is initiated by an oxidation reaction which generates reactive radicals from the pyrrole monomer. Radical monomers react with each other to form dimers and the subsequent oxidation of dimers and oligomers leads to chain growth of the polypyrrole polymer.

The oxidation reaction that initiates the polymerization of pyrrole can come from a number sources, such as electrochemical and chemical polymerization methods. In electrochemical polymerization, pyrrole is oxidized at the anode electrode in an electrochemical cell. This technique yields a thin polypyrrole film deposited onto the anode electrode. In the chemical polymerization method, a strong chemical oxidant is used to oxidize the pyrrole monomer. A polypyrrole precipitate is formed in this method. Polypyrrole in the form of thin films and powders is constrained to simple linear and bending actuation modes. Photopolymerization of polypyrrole is a promising technique to produce 3D structures that are capable of complex modes of actuation.

\subsubsection{Photosensitive polypyrrole formulations}

Several photosensitive polymer formulations exist for the conjugated polymer polypyrrole. The photopolymerization technique is best described as photochemically initiated polymerization since these polymer formulations use a weak oxidant metal salt which is a compound that has is unable to rapidly oxidatively polymerize the conjugated polymer in the formulation, but, the oxidation strength of this compound may be increased by external energy such as light to increase the rate of polymerization. This method is similar to chemical polymerization in that a polymer particulate is formed in the irradiated area opposed to a continuous polymer film as that is formed with the electropolymerization method. 
Step 1. Monomer Oxidation

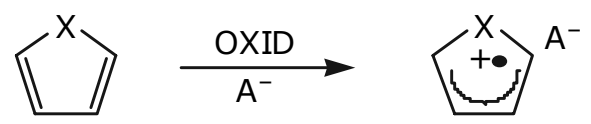

Resonance forms:

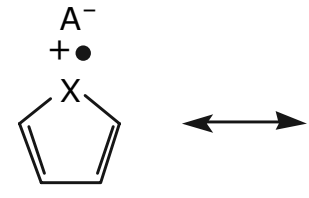

(I)

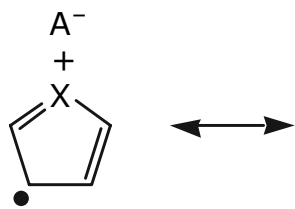

(II)<smiles>[Te][Te]1=CC=CC1</smiles>

(III)

Step 2. Radical-Radical Coupling<smiles>[Y7][Y]1=CC=C[C]1[C@H]1C=CC=[Y]1[CH]</smiles>

Step 3. Deprotonation/Re-Aromatization<smiles></smiles>

Step 4. Chain Propagation<smiles></smiles><smiles></smiles>

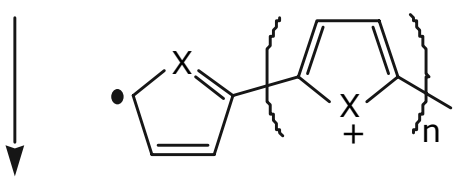<smiles></smiles>

Figure 2.8: The oxidative polymerization reaction for polypyrrole (@ Wallace et al., 2009, included with permission) 
The first formulations to photopolymerize polypyrrole utilized the light sensitive copper complex $[\mathrm{Cu}(\mathrm{dpp})]^{2+}(\mathrm{dpp}=2,9$-diphenyl-1,10-phenanthroline). The copper complex becomes excited when irradiated with UV light. When reacted with the electron acceptor p-nitrobenzyl bromide, the copper complex forms a strong oxidant to rapidly polymerize pyrrole Kern and Sauvage (1989).

The ruthenium complex $[R u(b p y) 3]^{2+}$ (bpy = bipyrideine) can be used to photopolymerize pyrrole in a similar mechanism Segawa et al. (1989). The UV excited state of the ruthenium complex is reacted with the electron acceptor $[\mathrm{CoCl}(\mathrm{NH} 3) 5]^{2+}$ to generate a strong oxidant to polymerize polypyrrole. This formulation has been further developed for the fabrication of 3D microscale structures within a support material using the twophoton polymerization technique Yamada et al. (2014).

Commercial photoinitiators containing an iron-arene structure, such as Irgacure 261 and Komplex KM 1144, are capable of photopolymerizing pyrrole. During photolysis these photoinitiators form a $\mathrm{Fe}(\mathrm{II})$ compound which is rapidly oxidized in air to $\mathrm{Fe}(\mathrm{III})$, a strong oxidant, to initiate the polymerization of pyrrole (Rabek et al., 1992b; Campomanes et al., 1999).

A well developed technique to photopolymerize pyrrole is with the use of a silver salt. $\mathrm{Ag}^{+}$ions like $\mathrm{Fe}^{3+}$ and $\mathrm{Cu}^{2+}$ are oxidants which can be used to chemically polymerize pyrrole. $\mathrm{Ag}^{+}$is however a weaker oxidant that takes several hours to chemically polymerize pyrrole. The oxidation reaction speed is greatly increased when the ion is excited by UV light. Silver salts like silver nitrate can be directly used as a photoinitiator for the photopolymerization of pyrrole due to its absorption peak at $220 \mathrm{~nm}$ (Mohandass et al., 2013). For use with longer wavelength light sources, a photoinitiator can be added to the formulation as an intermediate sensitizer (Murphy et al., 2001; Hodko et al., 2009). One of the advantages of silver salt based photosensitive formulations is that they produce a polymer with a high conductivity compared to other photosensitive formulations. The conductivity of silver salt formulations can be as high as $0.2 \mathrm{~S} \cdot \mathrm{cm}^{-1}$. 
Hodko et al. (2009) compared to $3 \cdot 10^{-6} \mathrm{~S} \cdot \mathrm{cm}^{-1}$ where the ruthenium complex is used (Segawa et al., 1989) and $3 \cdot 10^{-5} \mathrm{~S} \cdot \mathrm{cm}^{-1}$ when iron-arene photoinitiators are utilized (Rabek et al., 1992a). The improved conductivity is due to the formation of silver islands during the oxidative polymerization reaction where $\mathrm{Ag}^{+}$ions are reduced to silver metal (Kasisomayajula et al., 2016; Yang and Lu, 2005; Zang et al., 2016; Nazar et al., 2015).

A photoinitiating system isn't strictly needed to photopolymerize pyrrole. Studies by Rodríguez and González-Velasco (1990) have shown that pyrrole itself can be excited by UV irradiation to initiate photopolymerization. This self-sensitized polymerization technique however requires long irradiation times and produces a polymer with a low conductivity (Rodríguez and González-Velasco, 1990).

The photopolymerization technique has a unique capability to selectively polymerize pyrrole onto conducting or non-conducting substrates and has the potential to be integrated with the vat polymerization techniques to fabricate 3D conjugated polymer structures but, this method has some disadvantages. The major disadvantage of the photopolymerization technique is the poor electrical conductivity and mechanical integrity of the produced polymer. The product of photopolymerized pyrrole is a particulate, similar to the chemical polymerization method, making it difficult to create solid polymer structures of polypyrrole. When dried the polymer is brittle, limiting its applications in actuation technologies. Efforts have been made to improve their mechanical properties by incorporating large surfactant anions (Hodko et al., 2009) or simultaneous polymerization of pyrrole with another polymer (Asmussen et al., 2015; Migahed et al., 2004; Campomanes et al., 1999; Rinaldi et al., 2005; ljeri et al., 2010). Hybrid polymer systems allow the overall material to take on the smart material properties of polypyrrole and the mechanical properties of the secondary polymer. 


\subsection{Chapter summary}

This chapter has provided the necessary background information on additive manufacturing technologies and conjugated polymers from which this thesis builds upon. The unique properties of conjugated polymers are an attractive solution for advanced sensing and actuation applications, however, the poor processability of these polymers has hindered the development of this technology. The advancement of additive manufacturing has inspired the investigation of advanced fabrication techniques for this material which will allow researchers to take better advantage of its unique properties and ultimately open up new applications.

\section{List of references}

Asmussen, S., Arenas, G. and Vallo, C. (2015), 'Photopolymerization of pyrrole/methacrylate mixtures using $\alpha$-cleavage type photoinitiators in combination with iodonium salt', Synthetic Metals 209, 304-312.

ASTM (2015), 'Standard Terminology for Additive Manufacturing - General Principles - Terminology', ASTM International i, 1-9.

Beluze, L., Bertsch, A. and Renaud, P. (1999), Microstereolithography: a new process to build complex 3D objects, in 'Design, Test, and Microfabrication of MEMS and MOEMS', Vol. 3680, p. 808.

Campomanes, R., Bittencourt, S. and Campos, J. (1999), 'Study of conductivity of polypyrrol-poly(vinyl alcohol) composites obtained photochemically', Synthetic Metals 102(1-3), 1230-1231.

Choi, J.-W., MacDonald, E. and Wicker, R. (2010), 'Multi-material microstereolithogra- 
phy', The International Journal of Advanced Manufacturing Technology 49(5-8), 543551.

Cullen, A. T. and Price, A. D. (2018), 'Digital light processing 3D printing of intrinsically conductive polymer structures', Synthetic Metals 235(November 2017), 34-41.

Hafkamp, T., Van Baars, G., De Jager, B. and Etman, P. (2018), 'A Trade-Off Analysis of Recoating Methods for Vat Photopolymerization of Ceramics', Proceedings of the 28th Annual International Solid Freeform Fabrication Symposium pp. 687-711.

Hodko, D., Gamboa-Aldeco, M. and Murphy, O. J. (2009), 'Photopolymerized silvercontaining conducting polymer films. Part I. An electronic conductivity and cyclic voltammetric investigation', Journal of Solid State Electrochemistry 13(7), 10631075.

Holtrup, R. (2015), 'XZEED DLP. A multi-material 3D printer using DLP technology'.

ljeri, V. S., Nair, J. R., Gerbaldi, C., Gonnelli, R. S., Bodoardo, S. and Bongiovanni, R. M. (2010), 'An elegant and facile single-step UV-curing approach to surface nanosilvering of polymer composites', Soft Matter 6(19), 4666-4668.

Jacobs, P. F. (1992), Fundamentals of stereolithography, in 'Proceedings of the solid freeform fabrication symposium', pp. 196-211.

Jenkins, A. D. (2000), 'Photoinitiators for free radical cationic and anionic photopolymerisation', Polymer International 49(12), 1729-1729.

Joyce, M. (2017), 'Solid image apparatus with improved part separation from the image plate', US Patent 9,636,873 B2.

Kasisomayajula, S., Jadhav, N. and Gelling, V. J. (2016), 'Conductive polypyrrole and acrylate nanocomposite coatings: Mechanistic study on simultaneous photopolymerization', Progress in Organic Coatings 101, 440-454. 
Kengo Kobayashi and Koji Ikuta (2007), Advanced free-surface microstereolithography with $10 \mu$ resolution for hybrid microstructures, in '2007 IEEE/ASME international conference on advanced intelligent mechatronics', IEEE, pp. 1-6.

Kern, J.-M. and Sauvage, J.-P. (1989), 'Photochemical deposition of electrically conducting polypyrrole', Journal of the Chemical Society, Chemical Communications (10), 657-658.

Ligon, S. C., Husár, B., Wutzel, H., Holman, R. and Liska, R. (2014), 'Strategies to Reduce Oxygen Inhibition in Photoinduced Polymerization', Chemical Reviews 114(1), 557-589.

Liska, R. (2011), 'Industrial Photoinitiators: A Technical Guide. by W. Arthur Green.', ChemPhysChem 12(7), 1389-1389.

Mao, M., He, J., Li, X., Zhang, B., Lei, Q., Liu, Y. and Li, D. (2017), 'The emerging frontiers and applications of high-resolution 3D printing', Micromachines 8(4), 1-20.

Migahed, M., Fahmy, T., Ishra, M. and Barakat, A. (2004), 'Preparation, characterization, and electrical conductivity of polypyrrole composite films', Polymer Testing 23(3), 361-365.

Mohandass, C., Vijayaraj, A. S., Rajasabapathy, R., Satheeshbabu, S., Rao, S. V., Shiva, C. and De-Mello, I. (2013), 'Biosynthesis of Silver Nanoparticles from Marine Seaweed Sargassum cinereum and their Antibacterial Activity.', Indian journal of pharmaceutical sciences 75(5), 606-10.

Mueller, B. (2012), Additive Manufacturing Technologies, Vol. 32.

Murphy, O. J., Hitchens, G. D., Hodko, D., Clarke, E. T., Miller, D. L. and Parker, D. L. (2001), 'Method of forming electronically conducting polymers on conducting and nonconducting substrates', US Patent 6,210,537 B1. 
Nazar, R., Ronchetti, S., Roppolo, I., Sangermano, M. and Bongiovanni, R. M. (2015), 'In situ synthesis of polymer embedded silver nanoparticles via photopolymerization', Macromolecular Materials and Engineering 300(2), 226-233.

Pham, D. T. and Ji, C. (2003), 'A study of recoating in stereolithography', Proceedings of the Institution of Mechanical Engineers, Part C: Journal of Mechanical Engineering Science 217(1), 105-117.

Rabek, J. F., Lucki, J., Zuber, M., Qu, B. J. and Shi, W. F. (1992a), 'Photopolymerization of Pyrrole Initiated by the Ferrocene- and Iron-Arene Salts-Chlorinated Solvents Complexes', Journal of Macromolecular Science, Part A 29(4-5), 297-310.

Rabek, J. F., Lucki, J., Zuber, M., Qu, B. J. and Shi, W. F. (1992b), 'Polymerization of pyrrole by cationic photoinitiators', Polymer 33(22), 4838-4844.

Renap, K. and Kruth, J. (1995), 'Recoating issues in stereolithography', Rapid Prototyping Journal 1(3), 4-16.

Rinaldi, A., Kunita, M., Santos, M., Radovanovic, E., Rubira, A. and Girotto, E. (2005), 'Solid phase photopolymerization of pyrrole in poly(vinylchloride) matrix', European Polymer Journal 41(11), 2711-2717.

Rodríguez, I. and González-Velasco, J. (1990), 'Self-sensitized photopolymerization of pyrrole', Journal of the Chemical Society, Chemical Communications 104(5), 387388.

Sayao, K.-C. (2016), 'Stereolithography apparatus', US Patent 9,452,567 B2.

Segawa, H., Shimidzu, T. and Honda, K. (1989), 'A novel photo-sensitized polymerization of pyrrole', Journal of the Chemical Society, Chemical Communications 2(132), 132-133. 
Shin, D. G., Kim, T. H. and Kim, D. E. (2017), 'Review of 4D printing materials and their properties', International Journal of Precision Engineering and Manufacturing Green Technology 4(3), 349-357.

Smela, E. (2003), 'Conjugated polymer actuators for biomedical applications', Advanced Materials 15(6), 481-494.

Spinks, G. M., Alici, G., Mcgovern, S. and Xi, B. (2009), Section III Conjugated Polymer Actuators : Fundamentals.

Spinks, G. M., Alici, G., McGovern, S., Xi, B. and Wallace, G. G. (2009), Conjugated Polymer Actuators: Fundamentals, in 'Biomedical Applications of Electroactive Polymer Actuators', John Wiley \& Sons, Ltd., West Sussex, United Kingdom, pp. 193227.

Sun, C., Fang, N., Wu, D. M. and Zhang, X. (2005), 'Projection micro-stereolithography using digital micro-mirror dynamic mask', Sensors and Actuators, A: Physical 121(1), 113-120.

Tumbleston, J. R., Shirvanyants, D., Ermoshkin, N., Janusziewicz, R., Johnson, A. R., Kelly, D., Chen, K., Pinschmidt, R., Rolland, J. P., Ermoshkin, A., Samulski, E. T. and DeSimone, J. M. (2015), 'Continuous liquid interface production of 3D objects', Science 347(6228), 1349-1352.

Wallace, G. G., Teasdale, P. R., Spinks, G. M. and Kane-Maguire, L. A. P. (2009), Conductive electroactive polymers.

Wang, Y., Zhao, W., Ding, Y., He, Z. and Lu, B. (2009), 'A detection and control method of resin liquid-level of stereolithography apparatus', Rapid Prototyping Journal 15(5), 333-338. 
Yamada, K., Yamada, Y. and Sone, J. (2014), 'Three-dimensional photochemical microfabrication of poly(3,4-ethylene- dioxythiophene) in transparent polymer sheet', Thin Solid Films 554(2), 102-105.

Yang, X. and Lu, Y. (2005), 'Preparation of polypyrrole-coated silver nanoparticles by one-step UV-induced polymerization', Materials Letters 59(19-20), 2484-2487.

Zang, L., Qiu, J., Yang, C. and Sakai, E. (2016), 'Preparation and application of conducting polymer/Ag/clay composite nanoparticles formed by in situ UV-induced dispersion polymerization', Scientific Reports 6(1), 20470.

Zhou, C., Chen, Y., Yang, Z. and Khoshnevis, B. (2013), 'Digital material fabrication using mask-image-projection-based stereolithography', Rapid Prototyping Journal 19(3), 153-165. 


\section{Chapter 3}

\section{Development of a vat polymerization}

\section{system}

This chapter outlines the development of a vat polymerization additive manufacturing system for the fabrication of microscale conjugated polymer structures. A preliminary investigation into vat polymerization of conjugated polymer formulations was performed which provided valuable insight into the subsequent development of a custom designed high resolution additive manufacturing system. A detailed description on the designed system is presented with a discussion on the procedures used to calibrate the apparatus. Following chapters will use the systems outlined in this chapter to fabricate and characterize conjugated polymer structures. 


\subsection{Preliminary investigation into vat polymerization of conjugated polymers}

\subsubsection{Fixed-surface vat polymerization}

The initial attempt towards fabricating 3D conjugated polymer structures via the vat polymerization technique was pursued by making modifications to the commercially available B9Creator 3D printer. The B9Creator 3D printer utilizes a fixed surface vat polymerization technique. A slide peel release mechanism is used in this system to detach each layer from a PDMS window. While the slide peel release technique is an effective solution to reducing the peeling forces on the printed part, it requires a much larger polymer vat which is unfavourable for photosensitive conjugated polymer structures since the reagents in the formulation are expensive and spoil over several hours. As a result, the polymer vat shown in Figure 3.1, was designed to utilize the B9Creator with a reduced volume of polymer. The modified polymer vat featured a glass vat with either cast a PDMS or fluorinated ethylene propylene (FEP) film on the bottom to reduce peeling forces. A cylindrical aluminum rod was utilized as a build plate. Additional details on the design of the reduced volume polymer vat can be found in Appendix A.

The modified B9Creator was applied to the fabrication of conjugated polymer structures. The 3D printing process was able to proceed successfully for the first couple layers but it was found that due to the low stiffness of the conjugated polymer formulation, the structure eventually tore apart. Figure 4.4 shows an example of a failed print with one half of the structure attached to the build plate and the other on the window. The preliminary investigation into 3D printing conjugated polymers via the fixed-surface vat polymerization additive manufacturing found that the vat polymerization technique was not compatible with the low stiffness conjugated polymer formulation due to the 


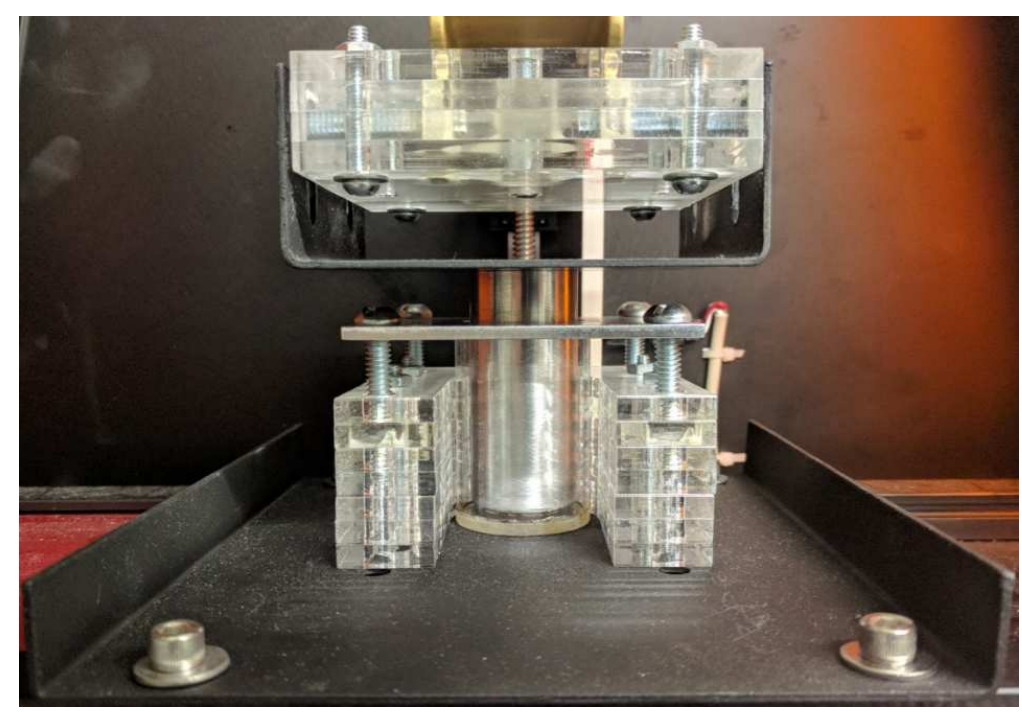

Figure 3.1: Modifications made to a B9Creator 3D printer to reduce the amount of photopolymer required in the photopolymer vat.

large peeling forces.

\subsubsection{Free-surface vat polymerization}

The free-surface vat polymerization additive manufacturing technique was investigated next as it has potential to overcome the challenge of working with low stiffness polymers. As discussed in Chapter 2, the free-surface technique cures thin polymer layers on top of the previously cured layer, eliminating the need to peel each layer from a window, making it ideal for fabricating structures with low stiffness photopolymers. The modifications shown in Figure 3.2 were made to the B9Creator to print with the freesurface vat polymerization technique. The apparatus consisted of a glass polymer vat contained within an air tight chamber with a window in the top. Oxygen inhibition at the curing layer was mitigated by purging the chamber with nitrogen gas. The liquid level within the polymer vat was controlled with a computerized syringe pump. To form an even polymer layer over the previously cured polymer layers, the vat was flooded until the surface tension was overcome to form uniform photopolymer layer. The photopolymer was drawn from the vat until the desired layer thickness was achieved. A DLP 


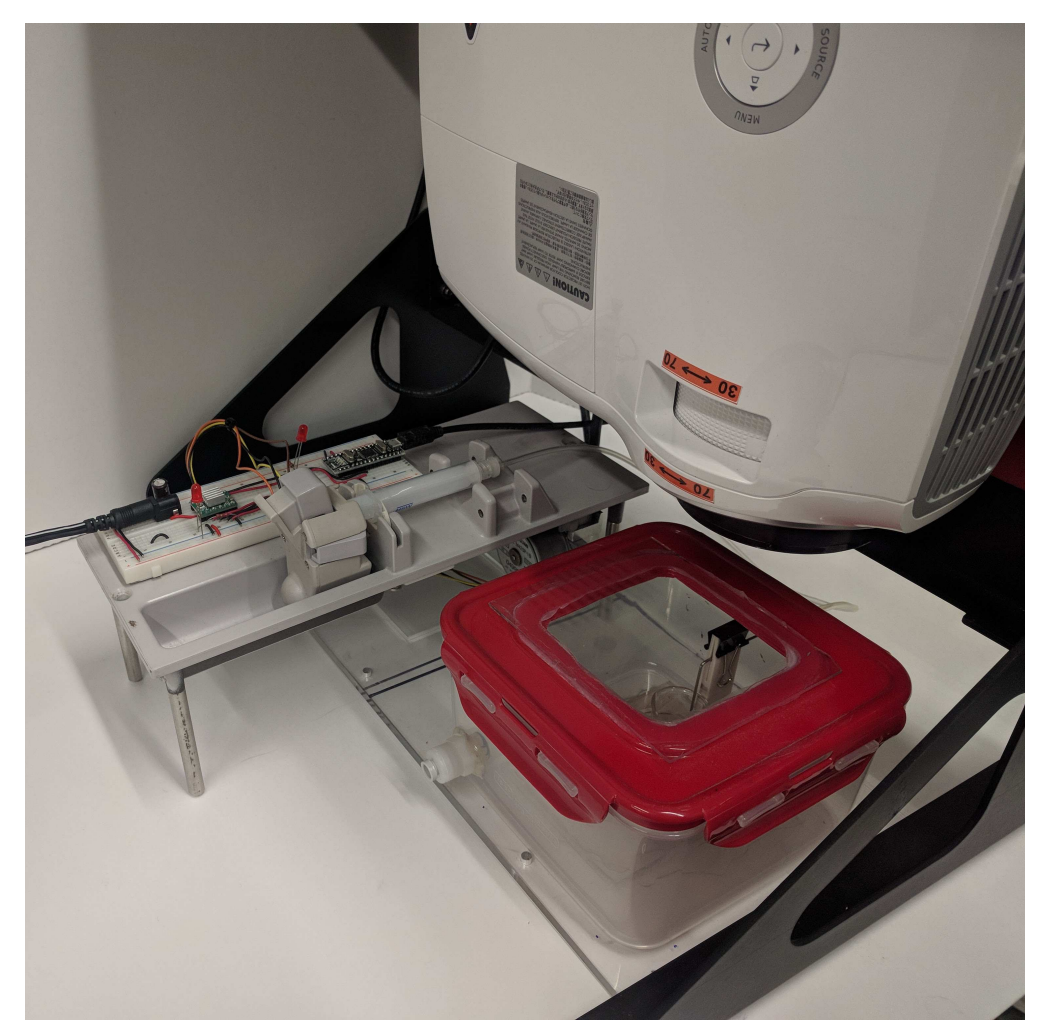

Figure 3.2: Modifications made to a B9Creator 3D printer to enable additive manufacturing with the free-surface vat polymerization technique.

projector was mounted above the chamber and projected the images of each layer through the window in the top of the chamber. A removable stainless steel sheet in the bottom of the polymer vat was used as a build surface. Additional details on the design free-surface vat polymerization modifications can be found in Appendix B. The millimetre scale conjugated polymer structures shown in Chapter 4.2.4 were fabricated with the free-surface vat polymerization technique, confirming that it is an effective method to produce 3D conjugated polymer structures.

The preliminary experiments with the fixed-surface and free-surface vat polymerization techniques provided valuable insight into the challenges of fabricating 3D conjugated polymer structures via vat polymerization. Based on the information gained from the preliminary experiments, a high resolution vat polymerization system was custom designed for the fabrication of 3D conjugated polymer structures. 


\subsection{Design of high resolution vat polymerization sys- tem}

\subsubsection{Design requirements}

The selection of an appropriate light engine for the high resolution vat polymerization system was carefully considered since it governed many of the design choices of the fabrication system. The DLP light engine utilized in the preliminary experiments was a standard projector with modified optics to produce an emission spectrum within the visible light spectrum. As a result, the selection of compatible photoinitiators was greatly limited. A specialized visible light photoinitiator was selected for the preliminary experiments which made it necessary to perform the polymer preparation under red light to prevent premature initiation. To expand the range of compatible photoinitiators for the photopolymer formulation and eliminate the inconvenience of preparing the polymer under limited light conditions, the light engine for the new vat polymerization system should have an emission spectrum within the UV spectrum. Due to the harmful nature of UV light, the projected light should be constrained within an enclosure with tinted windows.

The ability to fabricate micro-scale structures of conjugated polymers is desired as the response time of this electroactive materials is dependent on the diffusion of ions. Shoa et al. (2008) demonstrate that the speed of conjugated polymer actuators is dependent on the diffusion distance of ions through the material. To this end, the feature resolution of this high resolution vat polymerization system should be minimized to maximize actuator performance. The ability to fabricate micro-scale structures will allow this apparatus to create devices for applications within the fields of MEMS and microfluidics.

The amount of motion that can be produced with the volumetric expansion and 
contraction property of conjugated polymers can be enhanced with the use of passive structures. For example, the volume change of polypyrrole can be harnessed to produce large tip displacements when polypyrrole is coated onto a flexible substrate to form a bilayer actuator. In the fabrication of functional 3D printed conjugated polymer enabled devices, it will be desired to take advantage of the mechanical enhancement technique to improve the capability of produced devices. To achieve this feature, the additive manufacturing system must be capable of simultaneously printing active conjugated polymer structures and passive flexible structures. The ability to create active and passive structures will allow the passive structure of a device to be created around the active conjugated polymer components. For instance, this feature could enable the production of a microfluidic device with embedded conjugated polymer valves.

Within the preliminary experiments, a syringe pump system was utilized to laminate the photopolymer layers since this is one of the simplest laminating techniques. It was found that the syringe pump method was limited to accurately fabricating polymer layers as low as $50 \mu \mathrm{m}$. Due to backlash in the syringe pump design, pressure build up due to the viscous photopolymer formulation and surface tension between the photopolymer resin and the previously cured layer, the layer thickness could not be reduced further. The high resolution vat polymerization system will require a more advanced laminating technique to produce sufficiently thin layers for the desired feature sizes. The layer thickness should be close to the pixel resolution of the light engine.

\subsubsection{Design of DLP light engine setup}

The initial step in the design of the high resolution additive manufacturing system was to find a suitable DLP light engine. As discussed in Chapter 2, DLP light engines are best suited for vat polymerization systems due to the superior black level of the off pixels compared to LCD technology. Few commercial light engines are available that have a pixel resolution less than $20 \mu \mathrm{m}$. The PRO6500HR ultra-high resolution optical engine 
developed by Wintech Digital was selected for the system under development since this light engine is available with optics to produce a pixel size of $5 \mu \mathrm{m}$. This light engine utilizes the Texas Instruments DLP6500 device which has a pixel array of $1920 \times 1080$ to produce a maximum build size of $9.6 \mathrm{~mm} \times 6.4 \mathrm{~mm}$. The small build size of this light engine will be sufficient for the target MEMS and microfluidics applications since these devices are several millimetres in total size. The large image reduction in the optics of the light engine results in a small working distance of $30 \mathrm{~mm}$. This working distance restriction will make it challenging to maneuver a laminating system between the build surface and the optics of the light engine. As a result, the light engine is mounted to a linear stage to raise the optics of the light engine out of the way during the laminating procedure. The linear stage should have a high reliability to ensure the projected image is not distorted between subsequent layers. To meet this requirement, an OpenBuilds C-Beam linear stage was selected. Experimental results show that this linear stage has a repeatability of $80 \mu \mathrm{m}$ which is sufficiently accurate to yield an image distortion of less than $0.5 \%$. Figure 3.3 shows a computer aided design (CAD) model of the DLP light engine setup. The apparatus under development used a variety of optical breadboards to mount components in position. Custom brackets were machined to attach the non-standard components to the optical breadboards.

\subsubsection{Design of laminating system}

The dip and syringe pump laminating methods are desirable for use in free-surface vat polymerization systems since they require simple control techniques but the accuracy of the laminated layer decreases as the layer thickness is reduced due to surface tension forces with traditionally viscous photopolymers. To achieve layer thicknesses on the order of $5 \mu \mathrm{m}$ with a wide range of photopolymer formulations, the wiper laminating procedure was selected for the system under development. The laminating procedure

utilized in this system is shown schematically in Figure 3.4. After a 2D photopoly- 


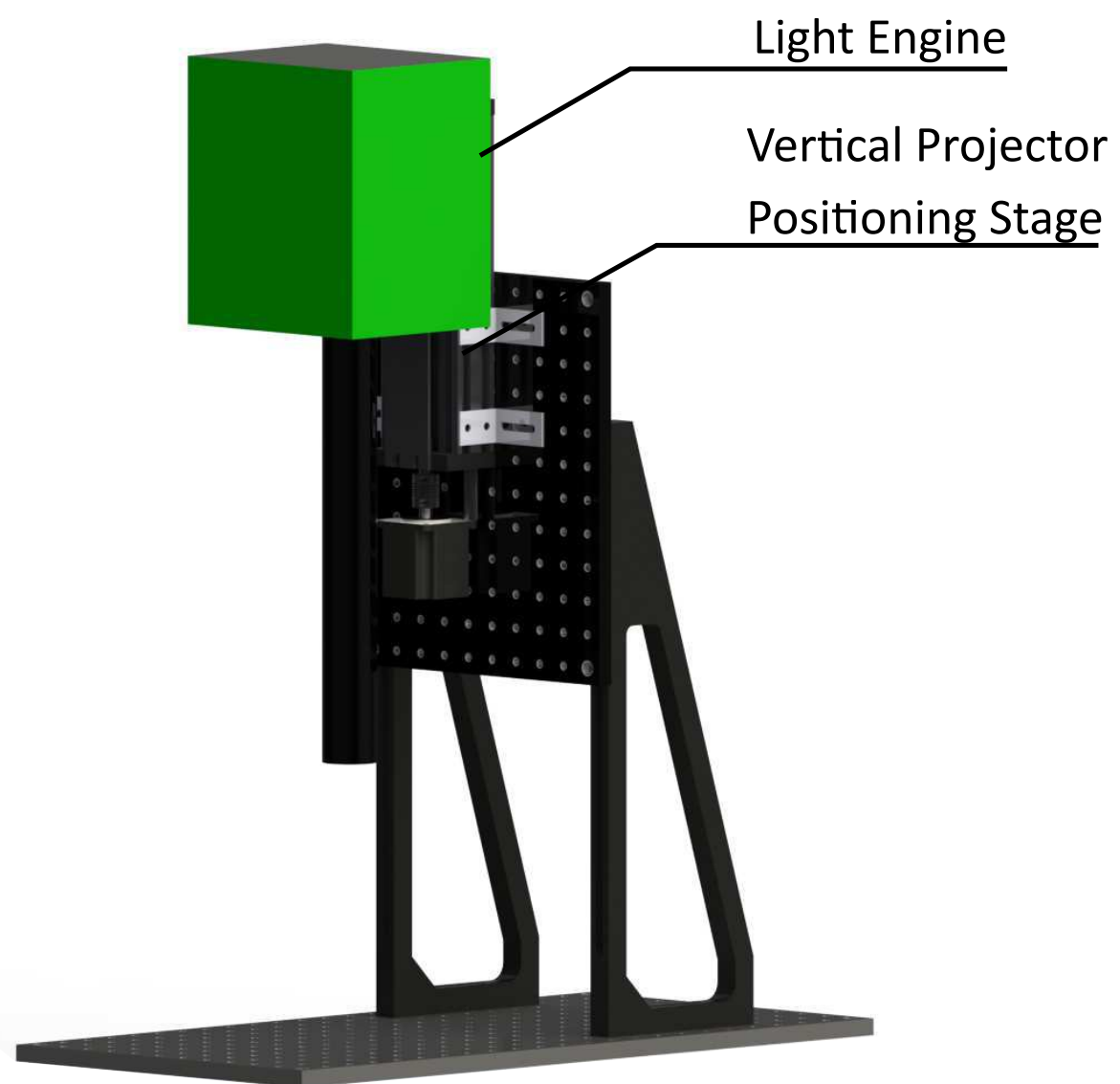

Figure 3.3: CAD model of the DLP light engine assembly featuring a PRO6500HR light engine mounted to a linear stage to move the optical assembly out of the way during lamination procedures. 
mer layer has been cured (Figure 3.4a), a non-contact laser displacement sensor first measures the height of the recently cured layer (Figure 3.4b), the build plate is then dipped into the photopolymer formulation (Figure 3.4c) and finally the wiper passes over the surface to form the final layer thickness (Figure 3.4e). The incorporation of a non-contact displacement sensor into the lamination procedure allows a consistent layer thickness to be formed as well as overcoming changes in the liquid level of photopolymer in the polymer vat which will arise from: polymer shrinkage when curing, volume displacement as the build plate is further lowered into the polymer vat and losses in photopolymer resin during the washing step of multi-material printing. Noncontact liquid level sensing is required instead of physical sensor technologies such as capacitive or float based sensors because the meniscus formed between the liquid photopolymer and a physical sensor will impact the minimum resolution of a physical liquid level sensor. A triangulation laser displacement sensor was selected for the liquid level measurement. This technology of sensor reflects a laser beam off target and the angle of reflection is measured with a charge-coupled device (CCD) sensor which is used to calculate the linear distance to the target. The ILD1420-10 laser displacement sensor was selected as it is one of the smallest devices on the market which reduces the distance the light engine has to move up, resulting in decreased fabrication time. The ILD1420-10 features a $10 \mathrm{~mm}$ measuring range, $0.5 \mu \mathrm{m}$ repeatability and an integrated controller which communicates the displacement reading via an RS-422 communication bus. Other non-contact displacement sensors such as ultrasonic and confocal were considered but the laser triangulation sensor was ultimately selected due to its compact size, high measurement repeatability, and lower cost. To meet the $5 \mu \mathrm{m}$ layer thickness requirement, the motion system for the wiper system must have a repeatability close to that of the linear displacement sensor. The L-509 series of precision linear stages from PI were selected since they feature a repeatability of $0.1 \mu \mathrm{m}$. This series of stages are easily mounted perpendicularly to achieve two axis motion, as shown 


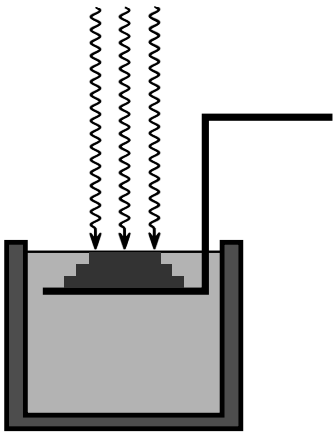

a) Cure Photopolymer

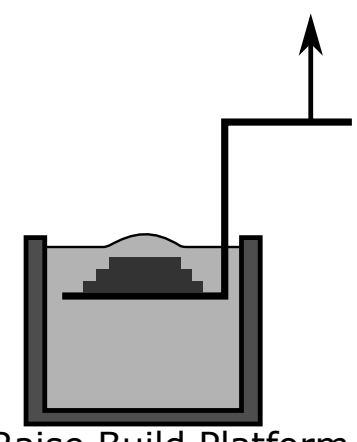

d) Raise Build Platform

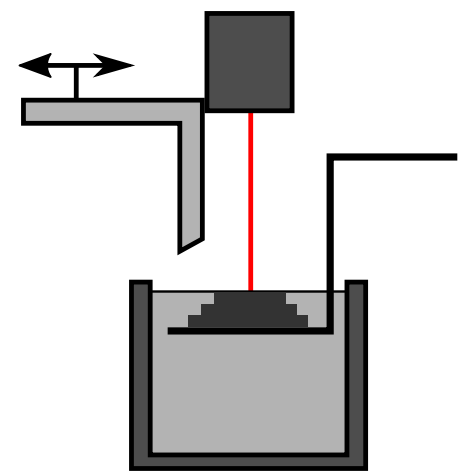

b) Measure Surface

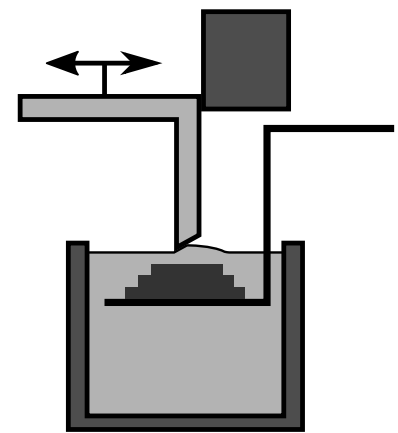

e) Wipe Layer

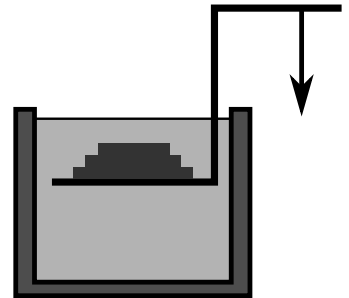

c) Lower Build Plate

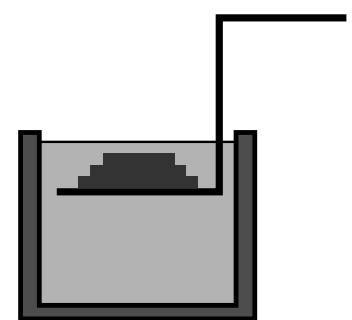

f) Prepare for Cure

Figure 3.4: Schematic representation of the lamination procedure used by the custom designed vat polymerization additive manufacturing system.

in Figure 3.5 depicting the laminating system. A L-509.10DG10 (geared DC motor drive with $26 \mathrm{~mm}$ travel range) was selected for the vertical axis and a L-509.20DG10 (geared DC motor drive with $52 \mathrm{~mm}$ travel range) was selected for the horizontal axis. A small manual stage was installed under the laminating system to provide perpendicular motion to the L-509 stages to align the wiper with the build plate while allowing the laser displacement sensor to be moved with a second axis over the build plate which is valuable when levelling the build plate.

\subsubsection{Design of multi-material system and build platform}

As discussed in Chapter 2, the volumetric expansion and contraction of conjugated polymer actuators is typically amplified with passive structures. A common example of 


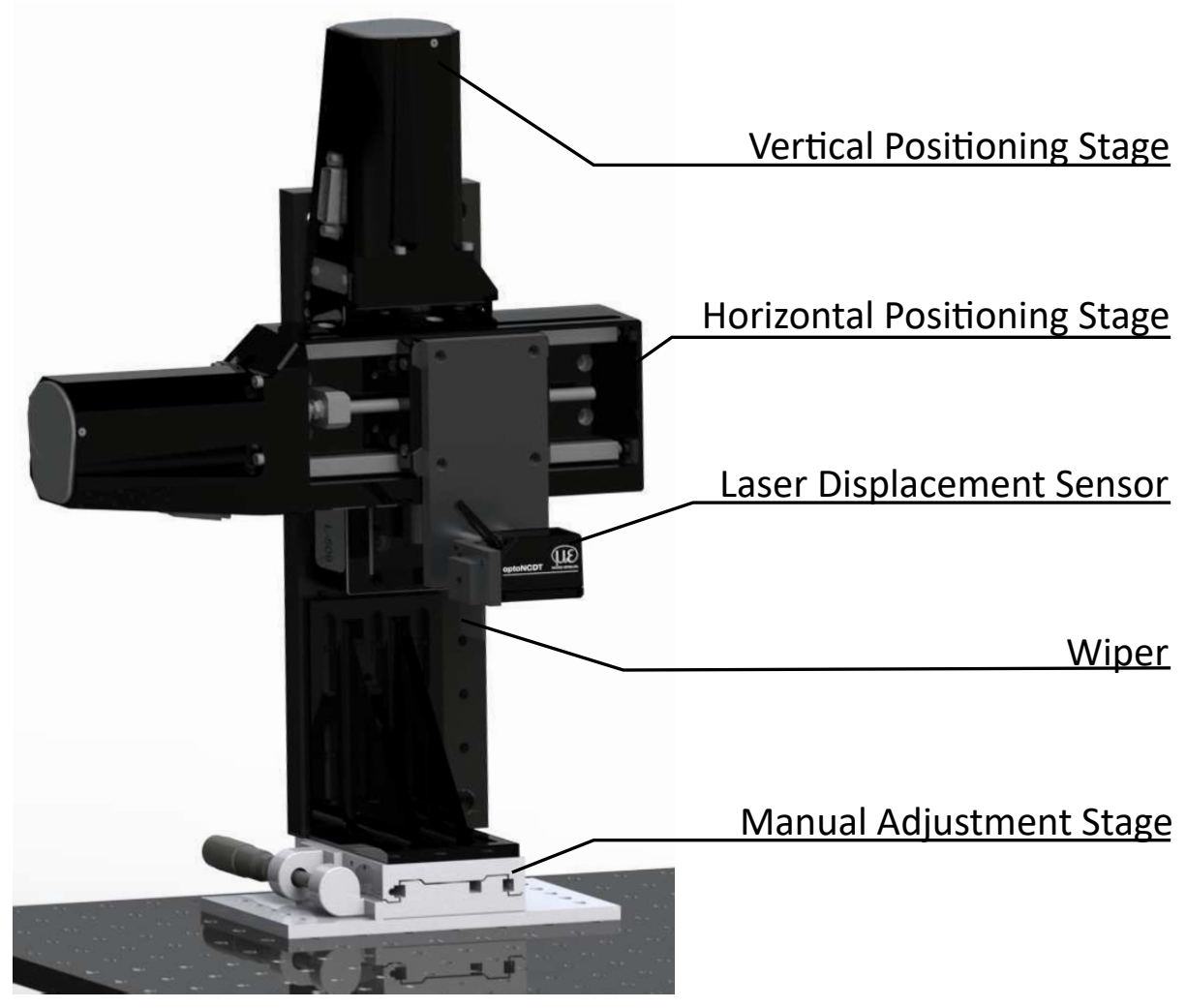

Figure 3.5: CAD model of the motorized laminating system which uses the wiper based lamination technique with an ILD1420-10 laser displacement sensor to measure the liquid photopolymer level in the photopolymer vat. 
this technique is with the bilayer actuator where the conjugated polymer is coated onto a thin compliant film to transform the volume change of the conjugated polymer to large tip displacements. To take advantage of this technique, the system under development will incorporate multi-material fabrication.

To incorporate the multi-material feature, the fabrication system will require 3 polymer vats, one containing a conventional photopolymer for passive structures, another for the active conjugated polymer and a final vat containing a solvent to wash the build plate when switching between polymer vats to prevent contamination. Figure 3.6 shows a 3D model of the designed multi-material system. A fourth station is present on the multi-material carriage which is used for calibrating the working distance of the light engine. Details on the working distance calibration procedure will be presented later in this chapter. Each station is fixed to a base plate with a laser cut alignment jig where the stations can be easily removed for filling and cleaning by removing a single bolt. The polymer vats are attached to an OpenBuilds C-Beam linear stage to automatically interchange the build plate material throughout the fabrication process. The accuracy of the multi-material stage does not have to be high as long as there is sufficient clearance between the build elevator and the walls of the polymer vat.

A sheet metal build plate elevator, shown in Figure 3.7 was designed to move the build surface in and out of the polymer vats. The build plate was sized to be $22 \times 12.5 \mathrm{~mm}$, which is larger than the maximum projected image size of $9.6 \times 6.4 \mathrm{~mm}$, to prevent non-uniformity at the edges of the build plate from affecting the build area. Magnets were installed in the build plate elevator to use an easily removable build surface with a flat surface finish. A 430 series stainless steel sheet with a smooth surface finish was used for the build surface due to its magnetic and corrosion resistance properties. The sheet metal build plate elevator is attached to a 5-axis alignment system which allows errors in the flatness of the component to be compensated for with a levelling procedure. The details of build plate levelling procedure will be discussed later in 


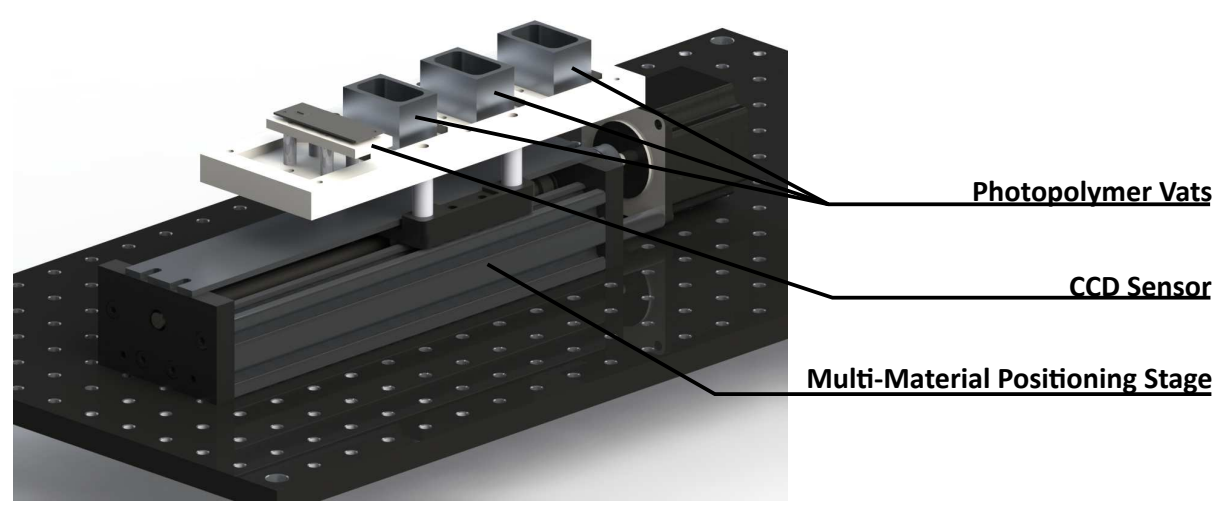

Figure 3.6: CAD model of the multi-material system which features three photopolymer vats attached to a linear to automatically interchange the material throughout the fabrication process.

this chapter.

Due to the confined space and required access around the polymer vat, an enclosed build chamber was not feasible. Instead, a steady flow of inert gas is passed over the build surface to prevent oxygen inhibition. The shielding gas system, shown in Figure 3.8 consists of a nitrogen inlet from a gas cylinder, an air flow regulator adjusted to $2 \mathrm{~L} / \mathrm{min}$, a solenoid valve, and a nozzle to direct the nitrogen gas over the polymer vat. A low flow rate was employed to prevent disturbances in the polymer surface.

\subsubsection{System control}

With many active components incorporated within the system under development, an embedded control system is required to convert the input fabrication files into actuations to produce the desired structures. The control system for the designed vat polymerization additive manufacturing system is summarized with the diagram in Figure 3.9.

Each of the PI L-509 precision linear stages is controlled by a dedicated C-863 DC motor controller. The three motor controllers communicate to each other in a daisy 


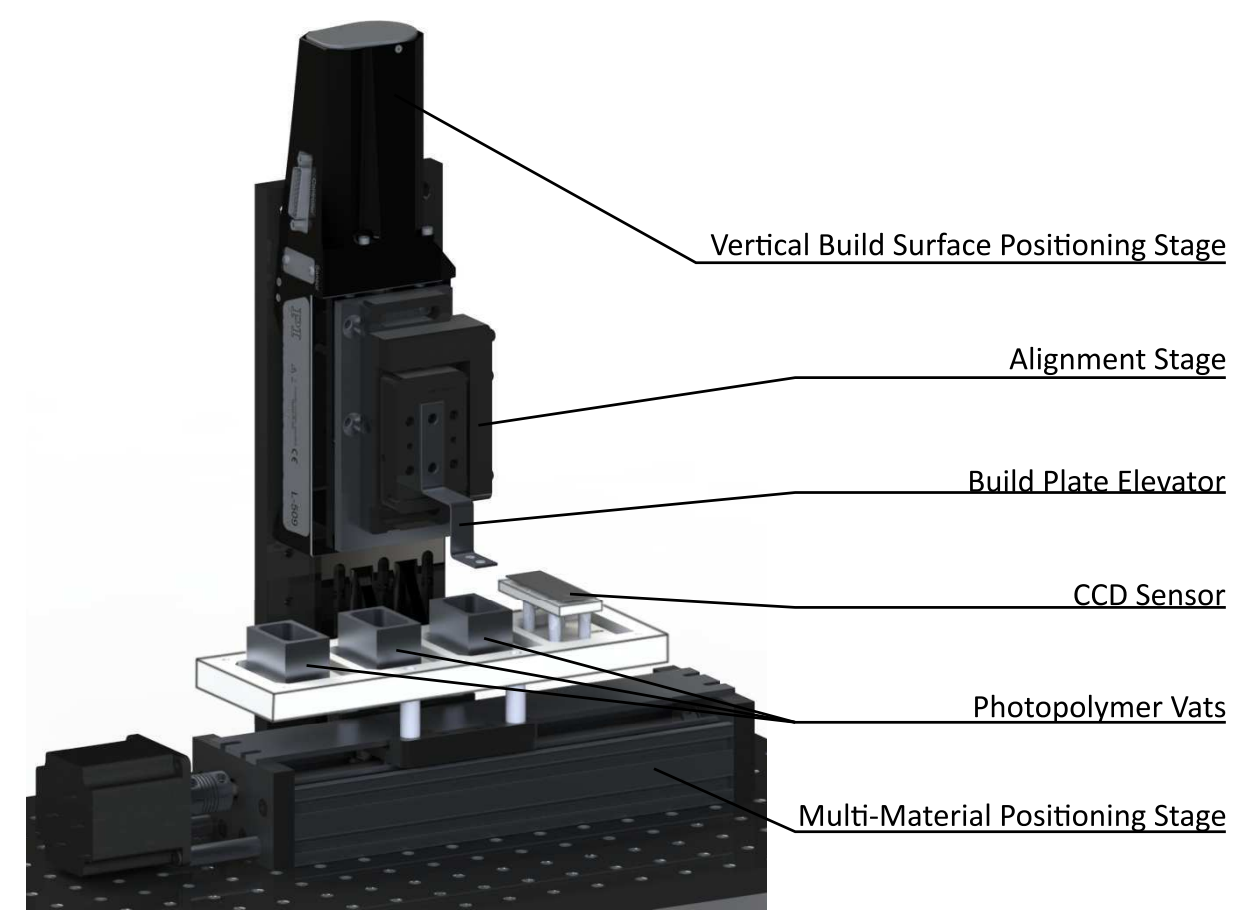

Figure 3.7: CAD model of the build plate system which features a magnetically detachable build surface attached to a 5-axis alignment system.

chain configuration via RS-232. A custom hardware communication board communicates to the head of the daisy chained motor controllers via RS232. The hardware communication board also communicates with the ILD1420 laser displacement sensor over RS422. The hardware communication board receives commands over an SPI bus. Each OpenBuilds C-Beam stage is controlled by a DQ542MA stepper motor driver.

Control signals to the DQ542MA stepper motor drivers, limit switches at the travel limits of the OpenBuilds C-Beam stages, pneumatic solenoid valve, SPI communication to the hardware communication board and emergency stop buttons are handed by a Smoothieboard numerical fabrication controller. The Smoothieboard is well suited for the system under development since it has a large assortment of inputs and outputs. The open source Smoothieware firmware that runs on the Smoothieboard is well documented and easily modified to make for the quick development of custom firmware.

A Raspberry Pi 2 running NanoDLP, a program to host and control DLP 3D printers, 


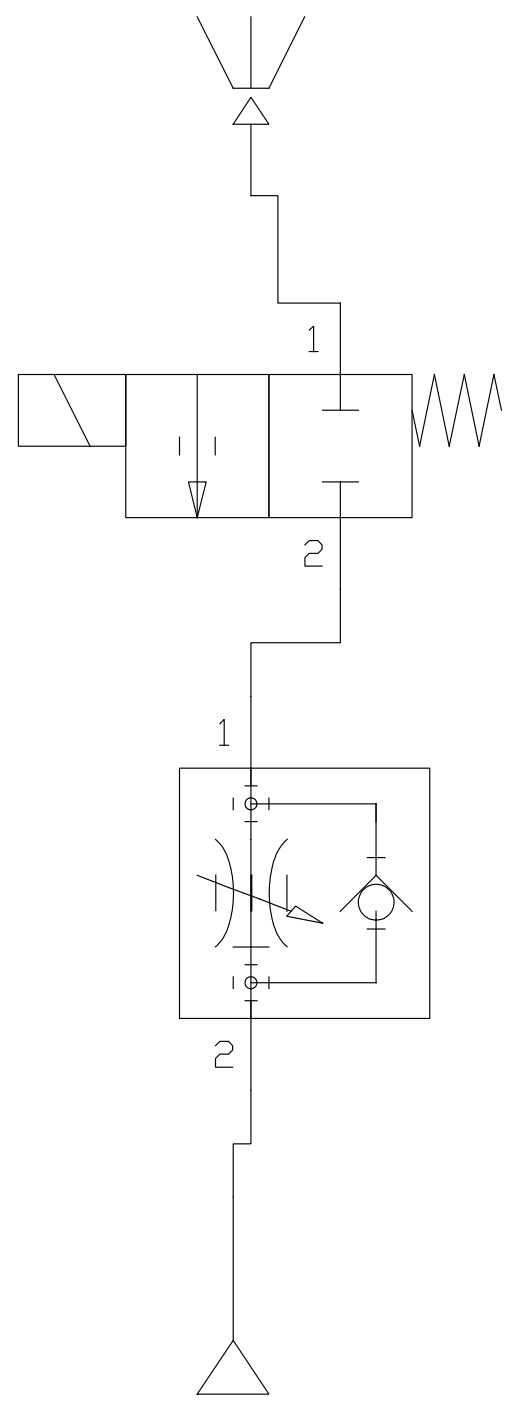

Figure 3.8: Schematic diagram of the shielding gas system used in the vat polymerization additive manufacturing system to prevent oxygen inhibition of the curing layer. 


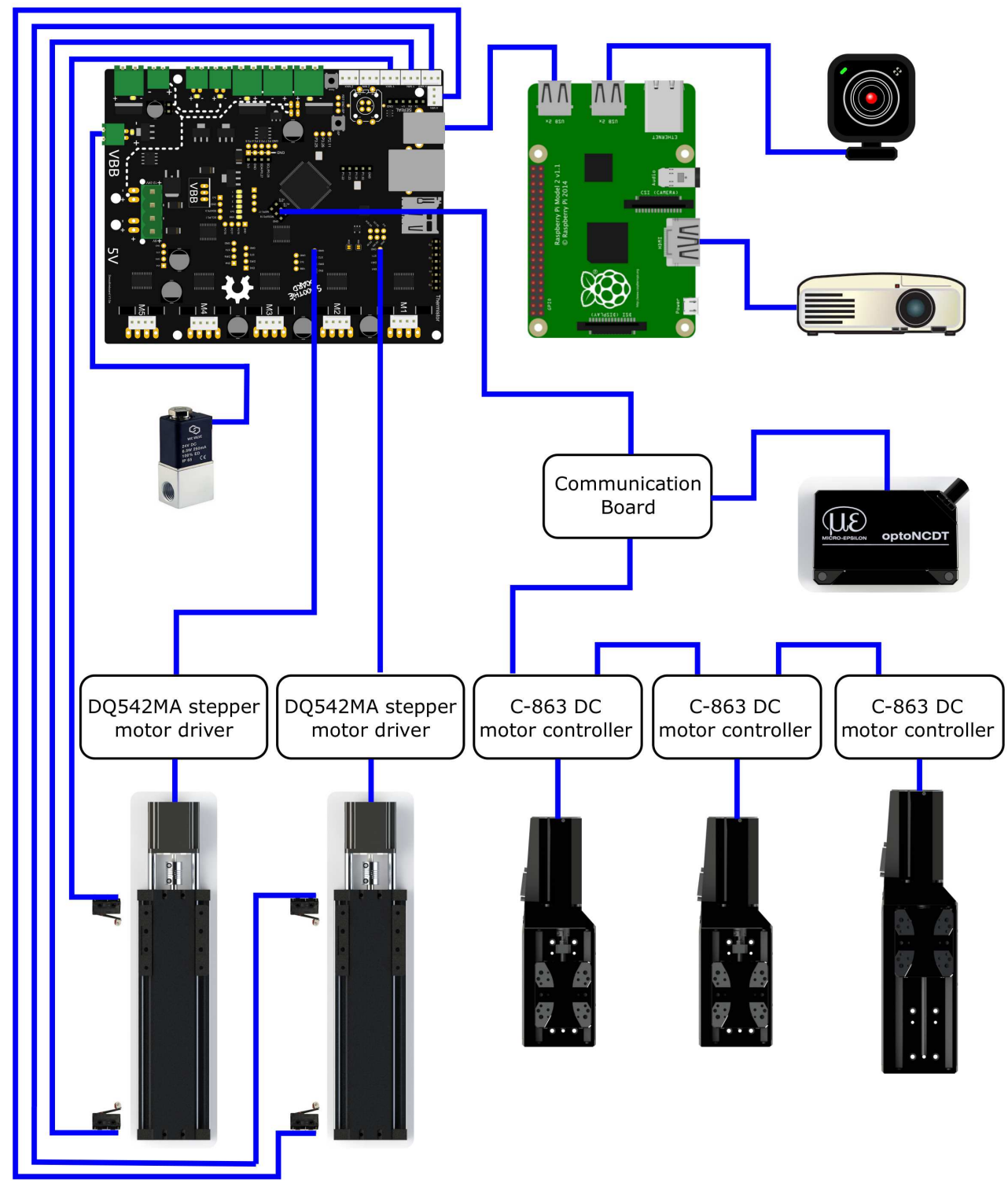

Figure 3.9: Schematic diagram of the control system used by the vat polymerization additive manufacturing system. 
is used to coordinate the additive manufacturing process. G-code commands are communicated to the Smoothieboard controller over USB from the host software. The CCD sensor used for projector focus detection is connected to a USB port on the Raspberry $\mathrm{Pi}$. Cross-section images of the part being produced are sent to the light engine over an HDMI port to selectively cure the photopolymer layers. The NanoDLP program running on the Raspberry Pi hosts a web application for the user to interface with the control software. The source code of the user interface was modified to add additional buttons for the user to manually control the components in the vat polymerization additive manufacturing system.

The workflow to create 3D structures with the designed vat polymerization additive manufacturing system begins with a 3D model of the desired part. The file is run through a slicing program to discretize the 3D geometry into thin $2 \mathrm{D}$ slices. The open source slicing program SLAcer was used for this project. SLAcer outputs a compressed folder containing a black and white image for each 2D slice in the 3D structure. The folder of images is input to the NanoDLP host software where it is combined with a resin profile containing information such as the desired layer thickness and the cure time for each layer. Once the additive manufacturing system has been calibrated and the desired material has been filled into the polymer vats, the 3D structure can be fabricated.

The entire apparatus is contained in an enclosure shown in Figure 3.10. The sides of the enclosure are made from tinted polycarbonate sheet to prevent both premature polymerization of the photopolymer during fabrication and the emission of potentially harmful UV irradiation. A sheet of polycarbonate was mounted vertically towards the rear of the enclosure to mount the electronics of the apparatus. A door was installed on both the front and rear of the enclosure to provide easy access to both the polymer vats and the electronics panel. The base plate of the apparatus was attached to the enclosure with vibration dampening standoffs to prevent vibrations from introducing 
errors into the fabrication process.

\subsection{Calibration procedures}

\subsubsection{Build plate levelling procedure}

The build plate of the fabrication system was attached to a 5 -axis alignment stage to ensure the build plate was flat to the photopolymer surface. By adjusting fine pitch set screws on the alignment stage, the orientation of the build platform could be adjusted to a maximum of $8^{\circ}$. The alignment stage allowed a sheet metal bending manufacturing method to be utilized to fabricate the build plate elevator since inaccuracies could be accounted for with a calibration procedure. The laser displacement sensor was utilized to measure the alignment of the platform. The calibration procedure consisted of measuring points on the build plate and adjusting the alignment of the stage until the height measurement between measurement points $5 \mathrm{~mm}$ apart were with $1 \mu \mathrm{m}$ of each other. The horizontal PI precision stage was used to move the measurement point across the width of the build platform, while the manual stage below the lamination system was used to move along the length.

\subsubsection{Liquid level measurement}

Before each fabrication job, the user must calibrate the height of photopolymer resin in the photopolymer vat and move the build plate elevator to the resin surface. The following steps are performed to calculate the required position of the build plate elevator:

1. The build plate is positioned above the desired photopolymer vat and the position is measured with the laser displacement sensor.

2. The build late is lowered to the bottom of the polymer vat. 


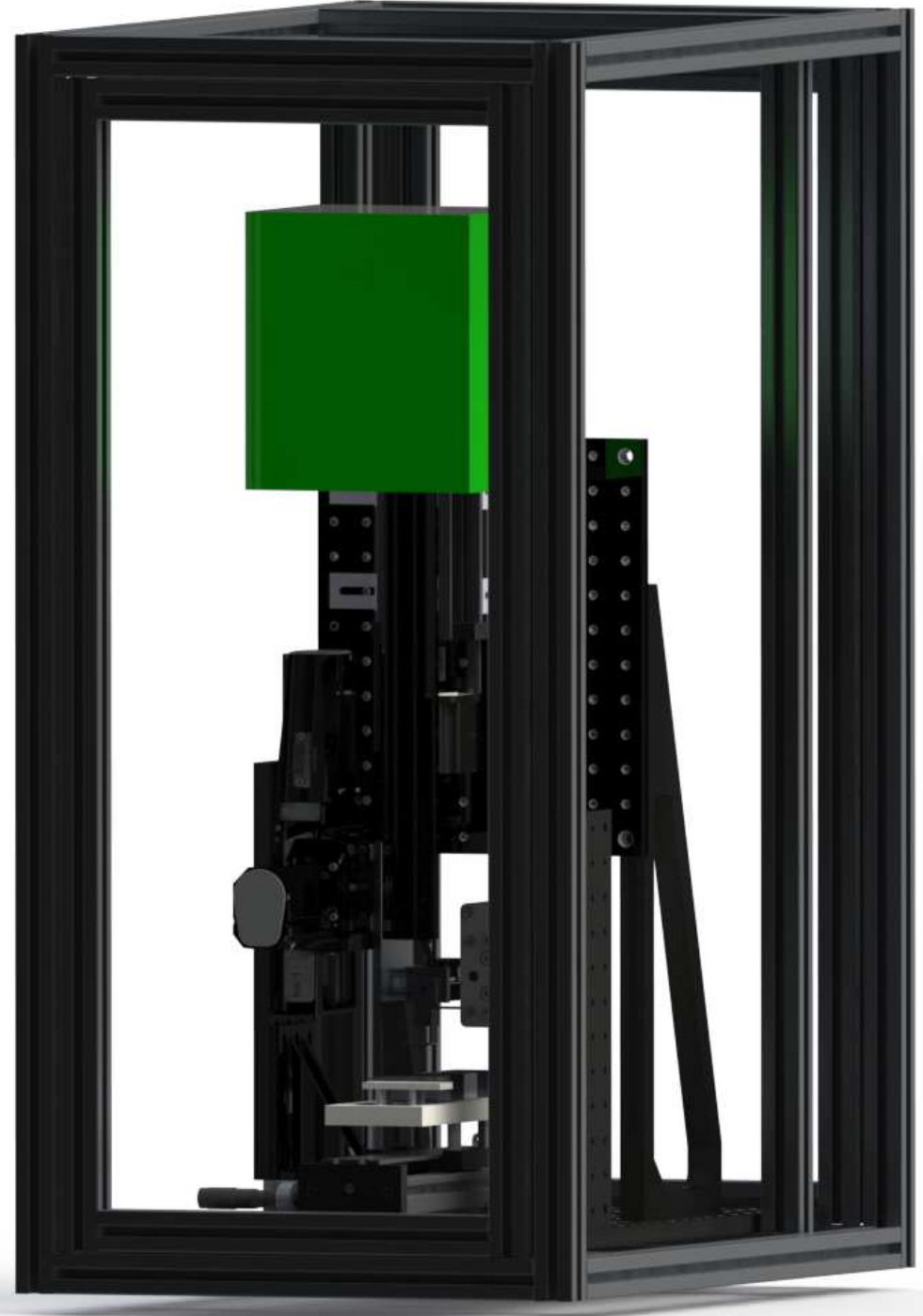

Figure 3.10: CAD model of the system enclosure to prevent external light from affecting the photopolymer during fabrication and releasing potentially harmful UV irradiation. 


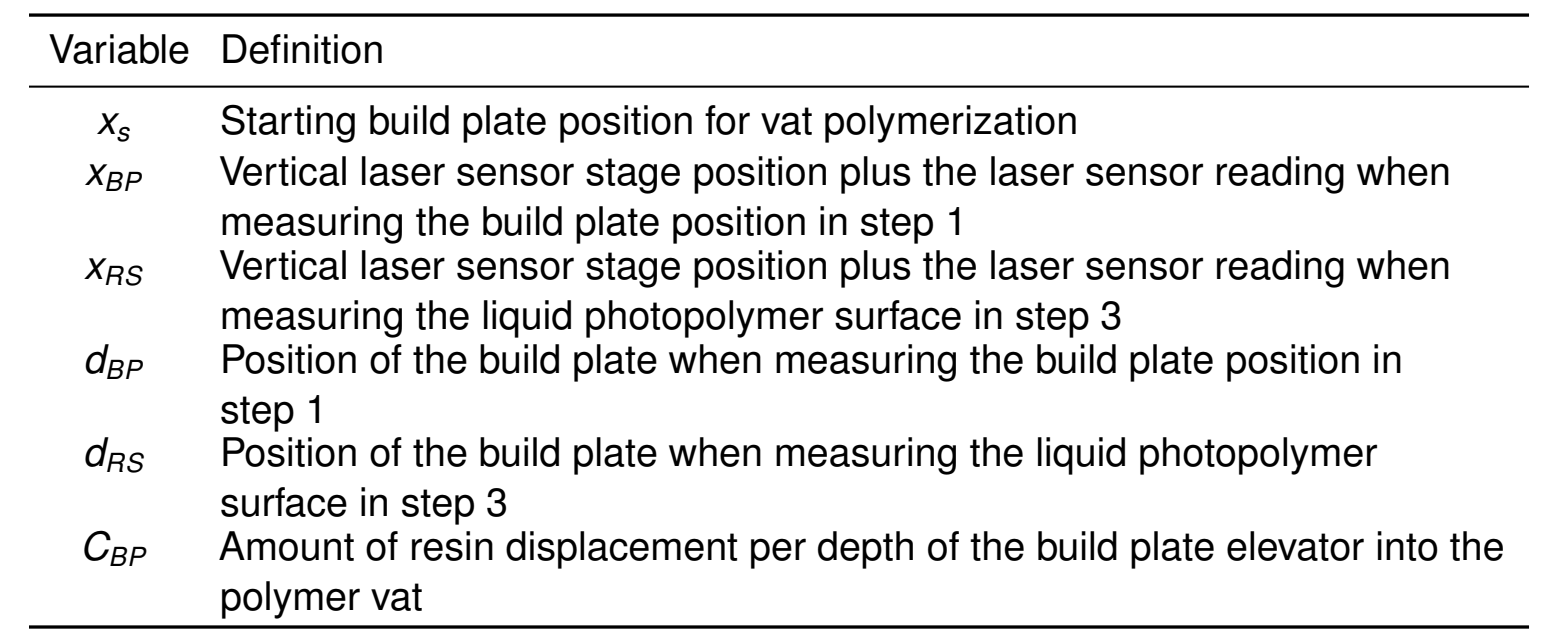

Table 3.1: Definition of variables in Equation 3.1 used to calculate the required build plate position

3. The laser displacement sensor is lowered and the position of the liquid photopolymer surface is measured.

4. The build plate elevator is moved to just below the liquid level.

The required position of the build plate is calculated with the following formula:

$$
x_{S}=\frac{x_{R S}-x_{B P}+d_{B P}-d_{R S} C_{B P}}{1-C_{B P}}
$$

The $C_{B P}$ term is used to compensate for the volume displacement that occurs due to the vertical section of the build plate elevator. The $C_{B P}$ constant was measured experimentally by measuring the photopolymer surface at various build plate positions below the resin surface. An extended initial cure time is used for the first couple layers of the fabrication process to compensate for errors in the calibration procedure and to ensure good adhesion to the build surface. 


\subsubsection{Light engine focal length measurement}

A fourth station was added to the multi-material stage for the purpose of calibrating the focal length of the light engine. This station features a CCD sensor which is used to detect the projected image from the light engine. The CCD sensor, shown in Figure 3.11 was made from a disassembled Logitech $\mathrm{C} 170$ with the lens removed from on top of the CCD sensor. The sensor is approximately $3 \times 2 \mathrm{~mm}$ in size with an image resolution of $640 \times 480$ resulting in a physical pixel size of approximately $4.5 \mu \mathrm{m}$ which is well suited for measuring the $5 \mu \mathrm{m}$ projected pixel size from the light engine. Based on the sharpness of the image projected onto the sensor, the focal length of the projector can be accurately calibrated. The following steps are performed in the calibration procedure:

1. The build plate is removed and the CCD sensor is moved to under the projector.

2. The laser displacement sensor is used to measure the position of the CCD sensor.

3. The light engine is moved to the approximate focal distance.

4. An image processing algorithm is run on the image detected by the CCD sensor and the light engine is moved up or down in a gradient descent algorithm.

Once the minimal point of the algorithm is reached, the position of the light engine stage and the position measured from the CCD sensor are recorded for use in the liquid level calibration procedure. The following formula is used to calculate the required position of the light engine stage:

$$
x_{p}=x_{f}+x_{R S}-x_{C C D}
$$

The image processing algorithm uses openCV to convert the image to grayscale, threshold the image, then sum the value of the pixels in the image. The smaller the 


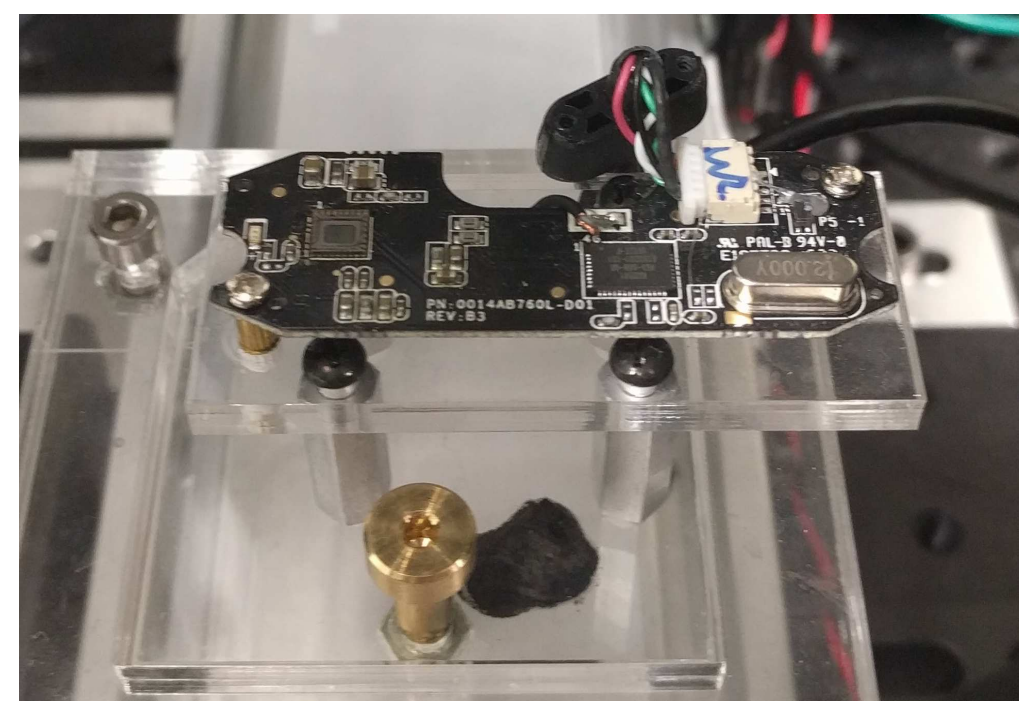

Figure 3.11: CCD sensor made from a disassembled Logitech C170 webcam which is used to calibrate the working distance of the light engine.

\begin{tabular}{cl}
\hline Variable & Definition \\
\hline$x_{p}$ & Projector stage position \\
$x_{C C D}$ & $\begin{array}{l}\text { Vertical laser sensor stage position plus the laser sensor reading when } \\
\text { measuring the CCD sensor position in step } 2\end{array}$ \\
$x_{R S}$ & $\begin{array}{l}\text { Vertical laser sensor stage position plus the laser sensor reading when } \\
\text { measuring the liquid photopolymer surface }\end{array}$ \\
$x_{f}$ & $\begin{array}{l}\text { Position of the projector stage when the image is in focus on the CCD } \\
\text { sensor }\end{array}$ \\
\hline
\end{tabular}

Table 3.2: Definition of variables in Equation 3.2 used to calculate the required projector position. 


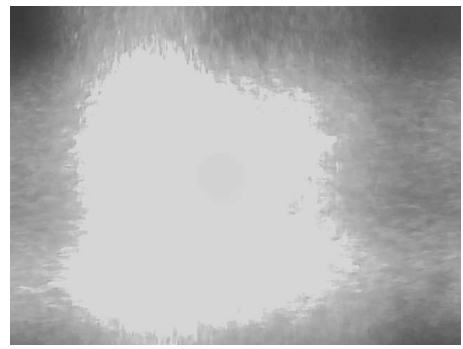

(a)

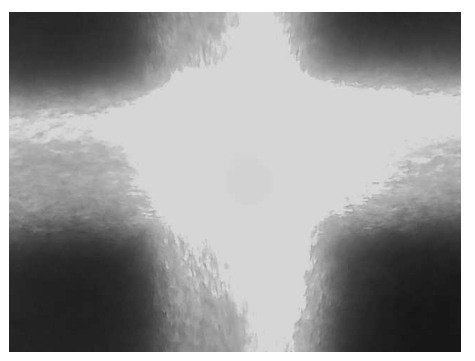

(b)

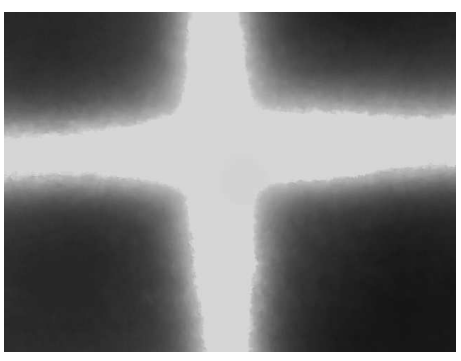

(c)

Figure 3.12: Collected CCD images from the light engine focal length measurement for when the projector is $5 \mathrm{~mm}(\mathrm{a}), 1 \mathrm{~mm}(\mathrm{~b})$, and $0 \mathrm{~mm}$ (c) away from the focal distance.

sum of the image, the greater the focus of the light engine. Figure 3.12 shows the processed CCD images for when the projector is $5 \mathrm{~mm}, 1 \mathrm{~mm}$, and $0 \mathrm{~mm}$ away from the focal distance.

\subsubsection{Settling time measurement}

The settling step present in the free-surface vat polymerization fabrication procedure is a critical step to the accurate and successful production of 3D structures. The structure shown in Figure 3.13 is an example of the defects that can occur when the settling time is too low. On the second layer of printing the pillar structures, the photopolymer layer was still settling when the next layer was being cured. The solidified photopolymer layer shifted before polymerization could propagate through the layer thickness to the previous layer, resulting in the formation tails on the pillars that point away from the center of the structure. Due to the high viscosity of photopolymer formulations and the thin layer thicknesses required to build 3D structures, the settling time in free-surface vat polymerization systems can take a considerable amount of the build time. The required settling time should be measured for each photopolymer and layer thickness.

To measure the settling time of a photopolymer resin, the ILD1420 laser displacement sensor was used to measure the liquid level of the photopolymer after the build plate elevator was moved to the required position to cure the next layer. The worst case 


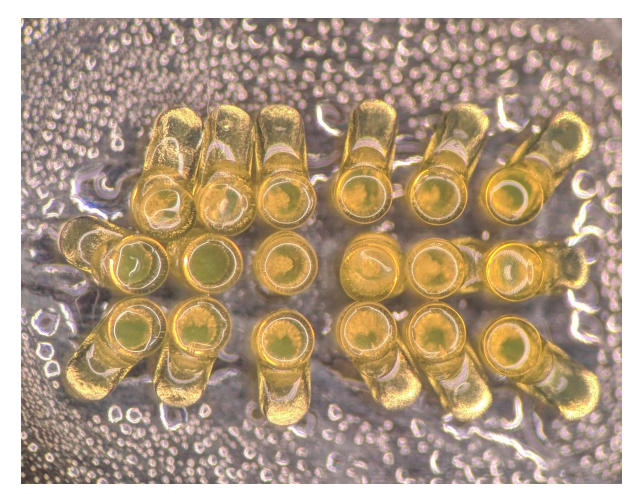

(a)

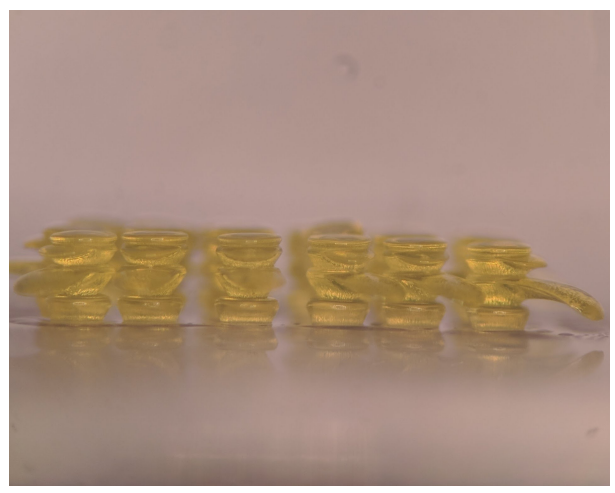

(b)

Figure 3.13: Failed multi-pillar fabrication job shows the type of error that can arise from the use of settling time that is too short.

settling time condition of a solid rectangle the size of the build area was used for the analysis since there is increased more fluid friction for the photopolymer to flow over the previously cured layer. Figure 3.14 depicts the liquid level over time for a $50 \mu \mathrm{m}$ layer of a yellow photopolymer from B9Creations. The settling time experiment reveals that a settling time of $30 \mathrm{~s}$ should be employed during the fabrication procedure.

\subsection{Fabrication of 3D microscale structures}

The performance of the custom vat polymerization system was validated with the fabrication the complex 3D structures with microscale structures made from a conventional photopolymer. Figure 3.15 shows a $6 \mathrm{~mm}$ tall chess rook with $50 \mu \mathrm{m}$ thick layers and Figure 3.16 shows a $2.5 \mathrm{~mm}$ tall standard benchmarking model with $25 \mu \mathrm{m}$ thick layers. Both of the print files present difficult structures for additive manufacturing system to produce but both were accurately fabricated after careful tuning of the 3D print parameters. 


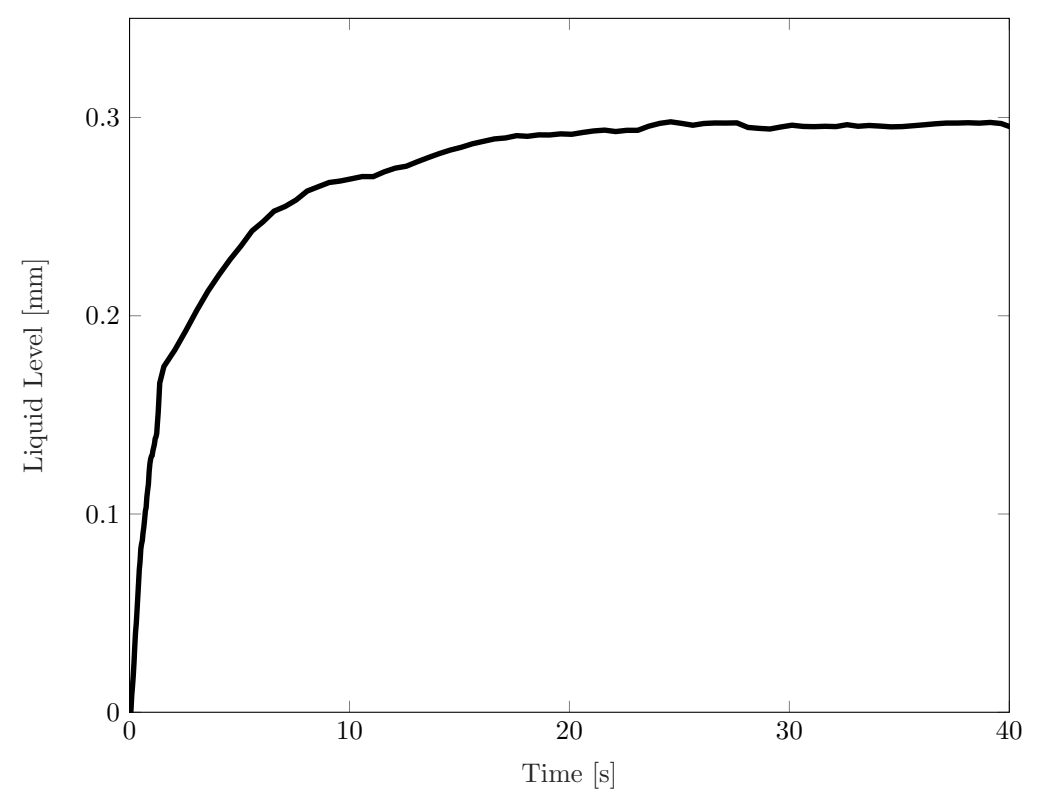

Figure 3.14: Height of the liquid photopolymer over time shows that $30 \mathrm{~s}$ are needed for the liquid photopolymer layer to settle.

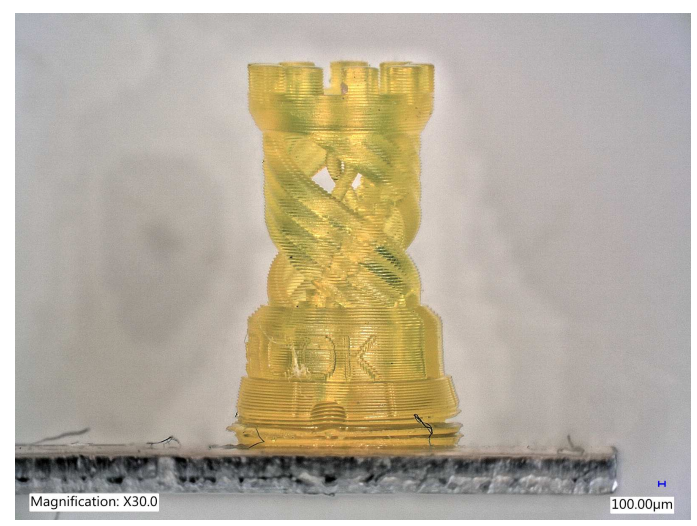

(a)

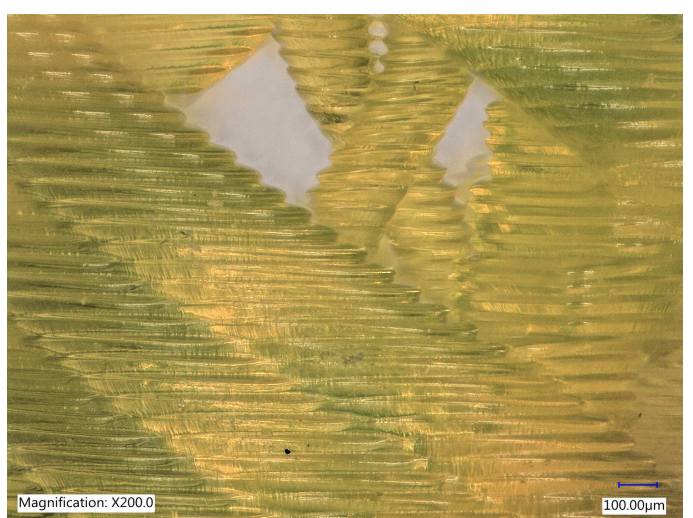

(b)

Figure 3.15: $6 \mathrm{~mm}$ tall chess rook with $50 \mu \mathrm{m}$ thick layers has fine features that are difficult for additive manufacturing systems to create. 


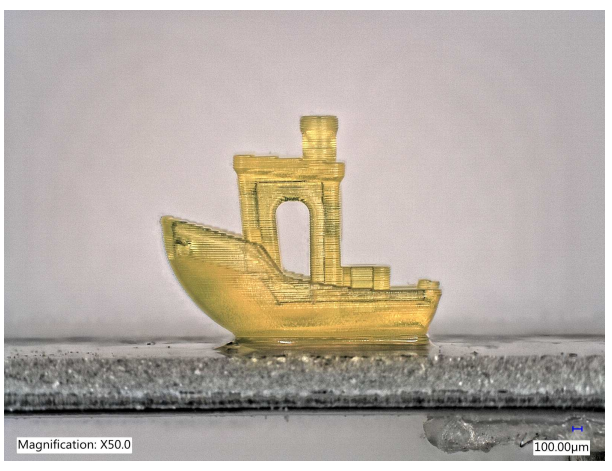

(a)

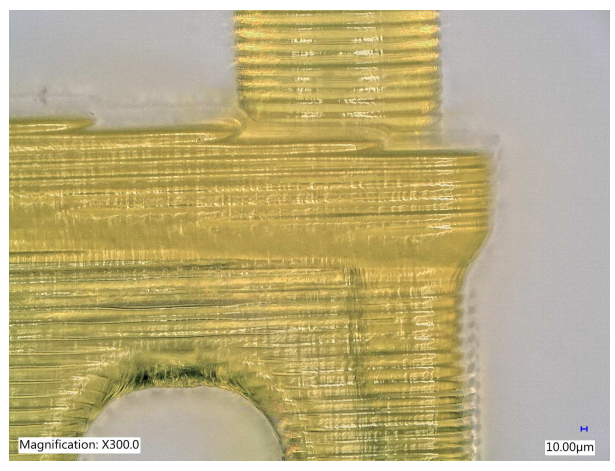

(b)

Figure 3.16: $2.5 \mathrm{~mm}$ tall standard benchmarking model with $25 \mu \mathrm{m}$ thick layers shows the type of difficult structures can be fabricated with the designed high resolution vat polymerization additive manufacturing system.

\subsection{Chapter summary}

This chapter presented the design of a vat polymerization additive manufacturing system for the fabrication of microscale conjugated polymer structures. Based on the results from a preliminary investigation into fabricating conjugated polymer structures with vat polymerization techniques, a subsequent high resolution vat polymerization system was designed utilizing the free surface approach with a non-contact displacement sensor, wiper based lamination system and multi-material capability.

\section{List of references}

Shoa, T., Madden, J. D., Fok, C. W. E. and Mirfakhrai, T. (2008), 'Rate Limits in Conducting Polymers', Advances in Science and Technology 61, 26-33. 


\section{Chapter 4}

\section{Development of a photosensitive conjugated polymer formulation}

This chapter outlines the development of a conjugated polymer formulation that is compatible with vat polymerization additive manufacturing. As introduced in Chapter 2, there have been a number of formulations developed for the photopolymerization of pyrrole. This research project focuses on photosensitive pyrrole formulations containing silver salts due to the relative simplicity of the photoinitiating system, and high electrical conductivities. A hybrid polymer formulation of polypyrrole and an acrylate based polymer was found to be an effective solution to improve the mechanical integrity of photopolymerized polypyrrole. The designed photosensitive formulations were characterized and applied to the fabrication of 3D conjugated polymer structures.

\subsection{Photopolymerization of a pyrrole-silver formulation}

\subsubsection{Design and characterization of a pyrrole-silver formulation}

The first investigation towards vat polymerization additive manufacturing of polypyrrole was with a formulation adapted from the work by Hodko et al. (2009) containing pyrrole, 
silver nitrate and a photoinitiator. An 8:1 molar ratio of pyrrole to silver nitrate was used since this ratio has been shown to produce polypyrrole with the greatest electrical conductivity (Hodko et al., 2009).

The pyrrole-silver formulations in this section were designed for use with the modified B9Creator as discussed in Chapter 3. The light engine used in the B9Creator is a commercial DLP projector with a spacer added to the lens assembly which allows the projector to have a shorter focal distance. The use of a commercial projector means the emission spectrum is within the visible light spectrum. To maximize the efficiency of the photoinitiating system, it should have a strong absorbance overlap with the emission spectrum of the light source. The $\mathrm{H}-\mathrm{Nu} 470$ radical photoinitiator was chosen for investigation due to its visible light absorbance up to $700 \mathrm{~nm}$ which has a strong overlap with the emission spectrum of the DLP projector. The absorbance spectrum of the $\mathrm{H}-\mathrm{Nu} 470$ photoinitiator and emission spectrum of the light engine are shown in Figure 4.1. The emission spectrum of the light engine and absorption spectrum of the photoinitiator were measured with a BLACK-Comet UV-VIS spectrometer. The absorbance spectrum of the photoinitiator was obtained from a $0.01 \mathrm{wt} \%$ solution of $\mathrm{H}-\mathrm{Nu} 470$ in propylene carbonate. The $\mathrm{H}-\mathrm{Nu} 470$ photoinitiator is known to be efficient and requires lower concentrations compared to UV photoinitiators (Marino et al., 1995). This photoinitiating system is known as radical sensitization of a cationic photoinitiator where the silver nitrate acts as the cationic photoinitiator (Bi and Neckers, 1994).

Preliminary experiments were performed with a polymer formulation containing an 8:1 molar ratio of pyrrole to silver nitrate and a $0.1 \mathrm{wt} \%$ concentration of the $\mathrm{H}-\mathrm{Nu} 470$ photoinitiator. The concentration of the photoinitiator in the polymer formulation was selected based on the manufacturer's recommendation. Due to the $\mathrm{H}-\mathrm{Nu} 470$ absorption of light within the visible spectrum, the polymer formulations were prepared under red light to prevent premature initiation. The polymer formulations were cured within a 1 dram glass vials purged with nitrogen gas to prevent oxygen inhibition. The polymer 


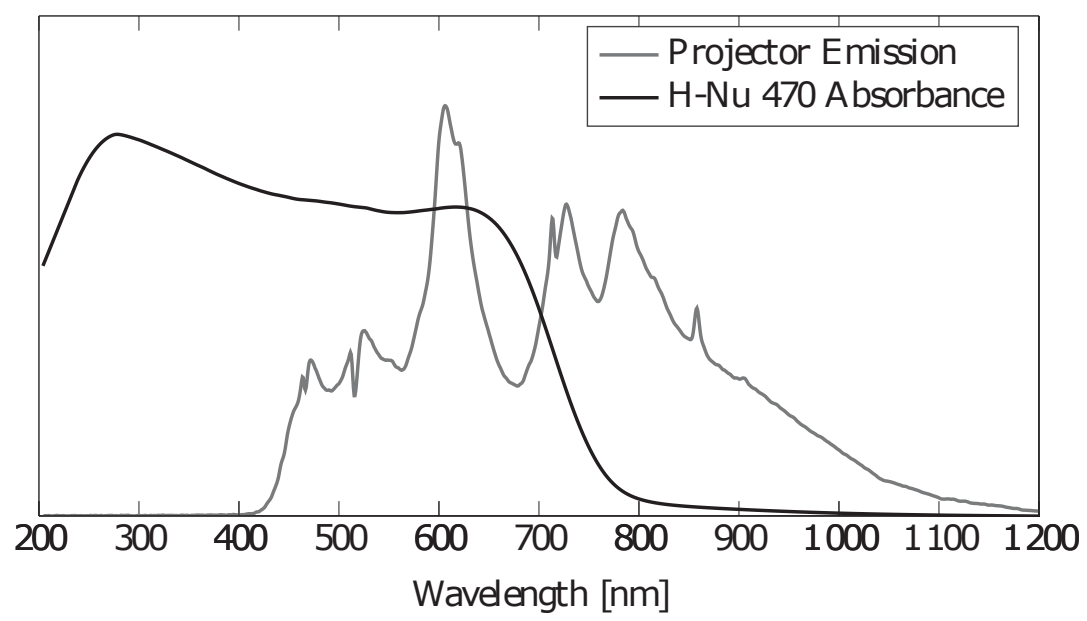

Figure 4.1: Emission spectrum of the projector and absorption spectrum of the $\mathrm{H}-\mathrm{Nu}$ 470 photoinitiator. The intensity and absorbance axes have been normalized.

formulation has a translucent amber appearance prior to curing and a characteristic dark black after curing. The colour change property of polypyrrole was used with timeresolved UV-VIS spectroscopy to evaluate the irradiation time required to photopolymerize pyrrole formulation. The experiment was performed by measuring the emission spectrum of the projector through the polymer formulation with a BLACK-Comet UV-VIS spectrometer equipped with a cosine receptor. The series of spectrograms in Figure 4.2 shows how the light passing through the polymer is almost entirely blocked after 20 min of irradiation. Figure 4.3 shows an image of the cured photopolymer. Cured polypyrrole has a black liquid appearance with a significant amount of particulate suspended in the solution. The surface of the vial in contact with the photopolymer became coated with the polymer particulate. The lack of a solid polymer structure presents a difficult challenge towards the fabrication of 3D conjugated polymer structures.

\subsubsection{Fixed-surface vat polymerization}

Despite the lack of a solid polymer structure, the polymer formulation was applied to the fabrication of $3 \mathrm{D}$ structures via vat polymerization additive manufacturing to gain further insight into the curing properties of the photosensitive polypyrrole formulations. The 
Time Resolved Spectrogram

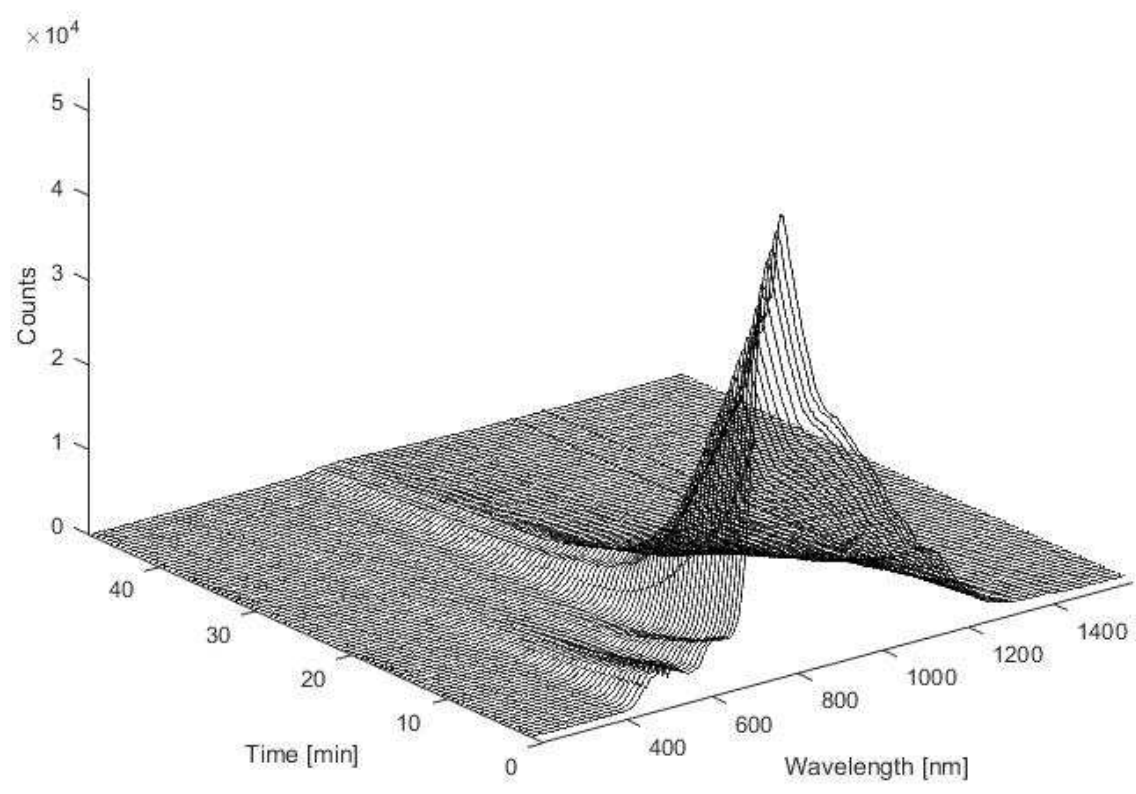

Figure 4.2: Time-resolved UV-VIS spectroscopy of the pyrrole-silver formulation throughout the polymerization reaction.

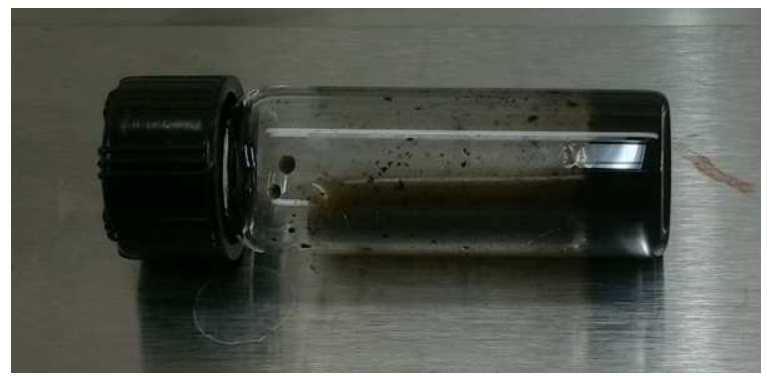

Figure 4.3: Glass vial containing the photopolymerized pyrrole-silver formulation indicates the presence of a polymer particulate. 


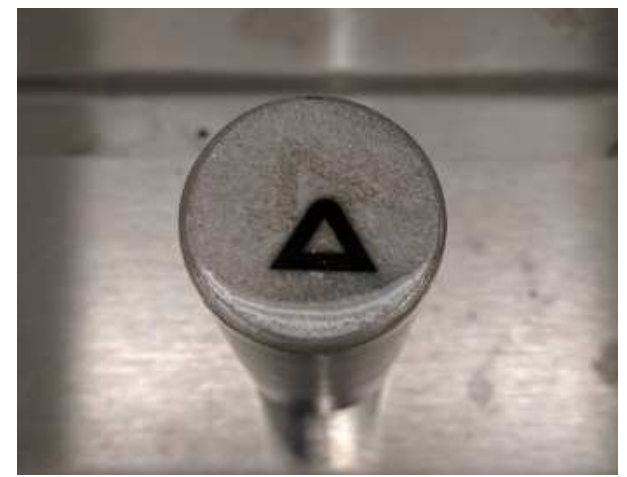

(a)

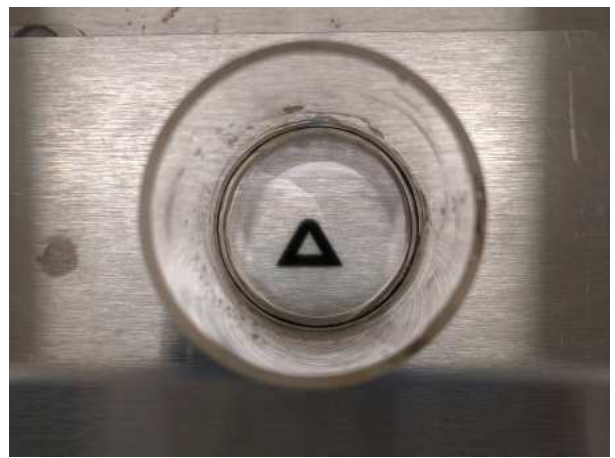

(b)

Figure 4.4: Failed 3D printed structure made from the pyrrole-silver photopolymer formulation where structure split into two halves with one half adhered to the build plate (a) and the other to the window in the polymer vat (b).

pyrrole-silver formulation was used with the preliminary fixed-surface vat polymerization apparatus discussed in Chapter 3 and detailed in Appendix B. Based on the timeresolved spectroscopy analysis, each layer of the 3D structure was cured for 20 min. The prototype apparatus was applied to the fabrication of a simple triangular structure which comprised of five $100 \mu \mathrm{m}$ layers. The fabrication procedure progressed as desired for the initial layers of the 3D structure but eventually, the peeling force split the structure into two halves with one half stuck to the build plate and the other to the window in the polymer vat as shown in Figure 4.4. Similar results were observed when the bottom of the glass polymer vat was lined with PDMS or FEP. It was concluded that the pyrrole-silver photopolymer formulation does not form a sufficiently stiff polymer to withstand the fabrication forces present with fixed-surface vat polymerization additive manufacturing.

\subsubsection{Free-surface vat polymerization}

As discussed in Chapter 2, an alternative technology to the fixed-surface technique is free-surface vat polymerization where the polymer layers are selectively polymerized on top of the previously cured layer. Due to the reduced fabrication force on the 


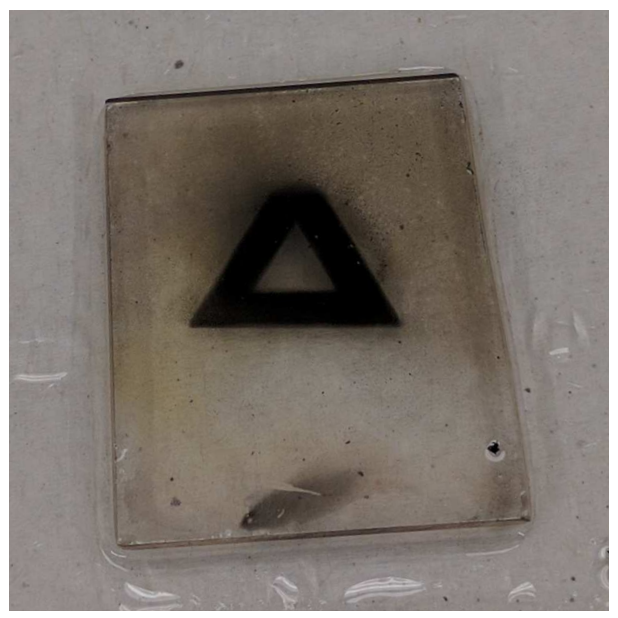

Figure 4.5: Triangular structure fabricated with the free-surface vat polymerization technique shows the poor curing properties of the pyrrole-silver formulation with the deposition of polymer particulates outside the irradiated areas.

produced structure, the free-surface technique was next investigated for the additive manufacturing with the pyrrole-silver photopolymer formulation. Details on the design of the prototype free-surface system are discussed in Chapter 3 and Appendix B. The prototype system was applied to the fabrication of the same triangular structure as with the fixed-surface apparatus. Figure 4.5 shows the produced structure built upon a glass substrate after three layers. During the fabrication process it was found that the cured photopolymer did not adhere itself to the previously cured layers but instead formed a particulate that flowed throughout the photopolymer vat as a new polymer layer was added to the vat. The polymer particulates can be seen in Figure 4.5 as a darkening of the glass build surface outside the irradiated areas. The deposition of polymer outside the irradiated areas increased as subsequent layer were cured.

\subsubsection{Conclusions on the pyrrole-silver formulation}

Based on the investigation into using the pyrrole-silver formulation with vat polymerization additive manufacturing systems, it has been concluded that the pyrrole-silver formulation does not form a sufficiently solid polymer structure upon curing to create 
layered 3D structures. The formation of a polymer particulate compared to the desired solid polymer is likely due to shorter polymer chain lengths arising from a lower strength oxidant compared to the strong oxidants used in chemical polymerization or the electric potential used in electropolymerization. To form solid 3D conjugated polymer structures with the photopolymerization approach, additional additives are required to improve the mechanical integrity of the produced polymer structures.

\subsection{Photopolymerization of pyrrole-UDMA hybrid poly- mer formulation}

\subsubsection{Design of pyrrole-UDMA hybrid polymer formulation}

Chapter 2 explained that one solution to the overcome the poor mechanical properties of photopolymerized PPy is the use of a hybrid polymer formulation. In this formulation, the hybrid polymer takes on the smart material properties of PPy and the mechanical properties of the secondary polymer. Based on the goal of applying the produced conjugated polymer structures to actuation technologies, the secondary polymer should be flexible since these devices require large volume expansion and contraction. To meet this need, a urethane dimethylacrylate (UDMA, Esstech Inc. X-726-0000) oligomer was selected for the secondary polymer since it is commonly used in photosensitive polymer formulations to yield a flexible polymer. Due to the flexible property of the cured polymer, the prototype free-surface vat polymerization system was utilized to prevent failure of the $3 \mathrm{D}$ structure during the peeling step. Various concentrations of UDMA in the photopolymer formulation were investigated. At high UDMA concentrations, the polymer formulation became viscous, making it difficult to dispense into thin layers. To compensate for this effect, the UDMA was diluted with $20 \mathrm{wt} \%$ propylene carbonate.

The hybrid polymer formulation requires a photoinitiating system to generate reac- 


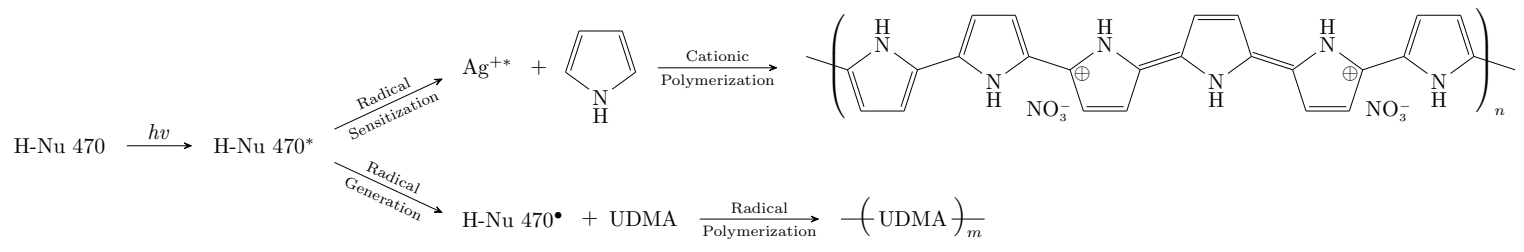

Scheme 4.1: Proposed reaction scheme of the two-component photoinitiating system for the pyrrole-UDMA polymer formulation.

tive species that initiate polymerization of both the pyrrole and UDMA monomers when irradiated with light. Since UDMA is known to polymerize with a radical photoinitiator (Yang et al., 2016), the $\mathrm{H}-\mathrm{Nu} 470$ and silver nitrate photoinitiating system used in the previous pyrrole-silver formulation may be used again. The $\mathrm{H}-\mathrm{Nu} 470$ photoinitiator will directly initiate polymerization of the UDMA polymer and will excite the silver ions to polymerize the pyrrole monomer. Reaction Scheme 4.1 summarizes the proposed two-component photoinitiating system. The same concentrations of $0.1 \mathrm{wt} \% \mathrm{H}-\mathrm{Nu} 470$ photoinitiator and 8:1 molar ratio of pyrrole to silver nitrate were used in this formulation.

Preliminary fabrication tests with $25 \%$ increment concentrations of the secondary urethane acrylate polymer indicated that a concentration greater then $75 \%$ was required to create a fast curing polymer matrix that maintained its integrity throughout the fabrication process. It was found that the UDMA polymer took less than two minutes of irradiation to form a solid polymer layer. The hybrid polymer formulation took advantage of the fast-curing UDMA to create a solid matrix that contained the pyrrole. The pyrrole was fully polymerized with a post-curing treatment. The difference in polymerization times between UDMA and pyrrole is apparent in the polymer's change in transparency. The hybrid polymer was initially translucent and with further irradiation, the polymer became an opaque black, which is characteristic of pyrrole. The fast curing matrix is required to fabricate $3 D$ structures with a high feature resolution because shorter irradiation times limit polymerization outside the irradiated areas. A photopolymer formulation containing 75 wt\% UDMA was used for the subsequent experiments. 


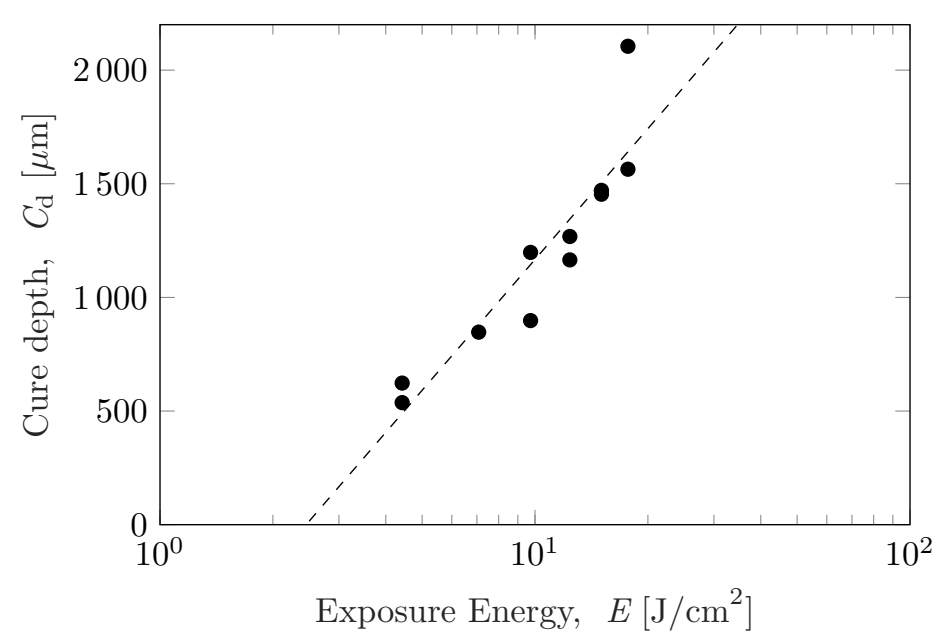

Figure 4.6: Depth of cure results for the pyrrole-UDMA polymer formulation.

\subsubsection{Depth of cure}

The reactivity of the polymer was evaluated with the depth of cure experiment described in Chapter 2. The experiment was performed by filling a cavity with the photopolymer resin and placing glass slide on top. The DLP light engine projected a circle on the photopolymer for a prescribed amount of time. After exposure, the polymer was washed with acetone to the remove uncured polymer and left to dry. The depth of polymerization was measured with an optical microscope. The working curve for the photosensitive PPy formulation containing $75 \mathrm{wt} \%$ UDMA is shown in Figure 4.6. The working curve of the photopolymer enables the selection of an appropriate irradiation time for a specified layer thickness when building multi-layered 3D structures.

\subsubsection{Minimum feature resolution}

The effect of the irradiation time on the minimum achievable feature size was evaluated through the fabrication of lines with a width of one pixel using increasing amounts of light energy. Larger rectangles were created at the ends of the fine parallel lines to anchor the fine structures to the build plate. The pattern used for this experiment is shown in Figure $4.7 \mathrm{a}$. The width of the produced lines was measured optically with a micro- 


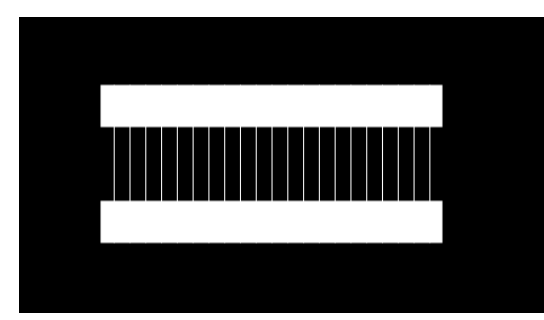

(a)

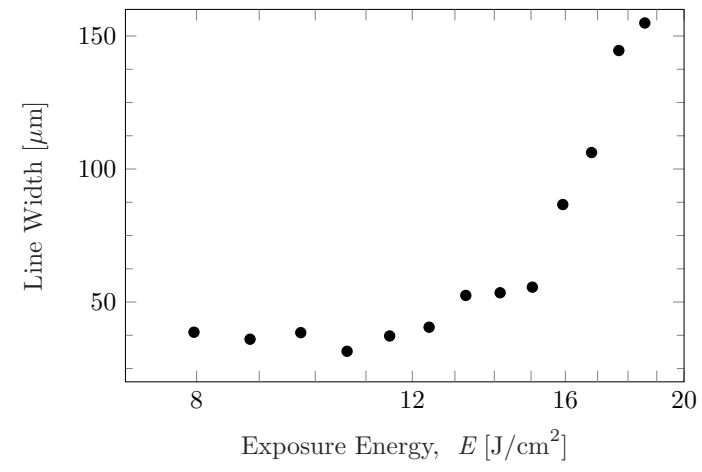

(b)

Figure 4.7: (a) Mask used for the feature resolution experiment and (b) the experimental results from the feature resolution experiment using the pyrrole-UDMA polymer formulation.

scope. This experiment was also used to validate the working curve from the depth of cure experiment since the smallest produced feature should have been irradiated with energy predicted by the working curve. The thickness was measured by mounting the build plate vertically and optically measuring the thickness via an optical microscope. Figure $4.7 \mathrm{~b}$ depicts the line-width results for a layer thickness of $98 \mu \mathrm{m}$ with a plot of line width versus irradiated light energy.

These results show that the line width initially increases slowly with increasing irradiation, yielding a width close the desired $40 \mu \mathrm{m}$ line width, but diverges quickly with irradiation energies greater than $12.5 \mathrm{~J} / \mathrm{cm}^{2}$. This property is common with photosensitive polymer formulations, illustrating the importance of accurate characterization of the reactivity of the polymer to produce features of the desired size. A minimum irradiation energy of $7.96 \mathrm{~J} / \mathrm{cm}^{2}$ was required to fabricate the fine feature which is $288 \%$ larger than the amount predicted by the working curve equation. This difference was attributed to additional polymerization required to adhere the structure to the build plate. The amount of additional curing is expected to be reduced when curing the polymer onto a previous polymer layer since the polymer will have a stronger bond to itself compared to the stainless steel build plate. 


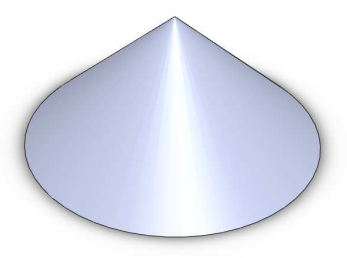

(a)

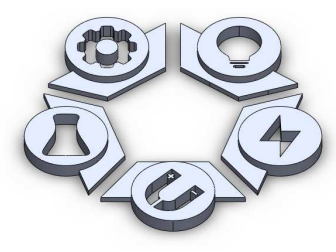

(d)

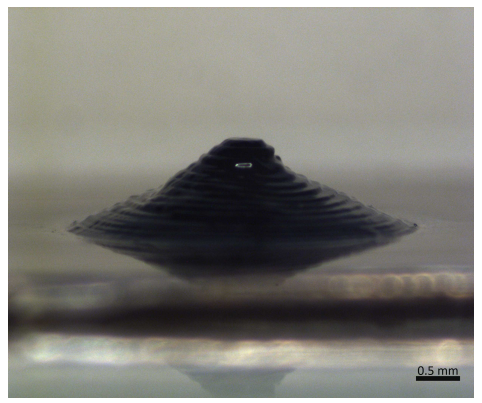

(b)

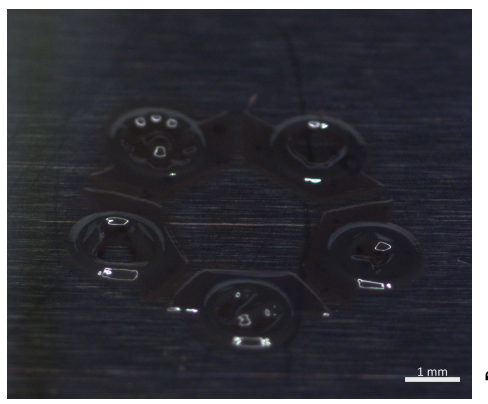

(e)

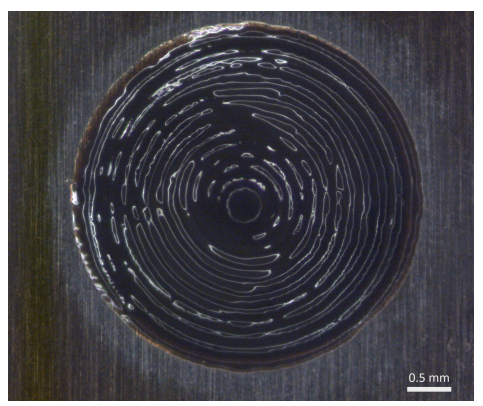

(c)

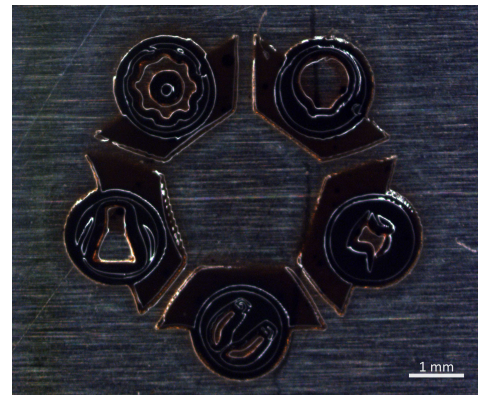

(f)

Figure 4.8: Fabrication of 3D conjugated polymer structures with $100 \mu \mathrm{m}$ thick layers. (a) and (d) depict geometric models of a 3D cone and logo respectively. (b) and (c) show optical images of the fabricated 3D cone. (e) and (f) show optical images of the fabricated logo.

\subsubsection{D Structure Fabrication}

The results from the depth of cure and minimum feature resolution experiments were used for the fabrication of 3D conjugated polymer structures. A layer thickness of $100 \mu \mathrm{m}$ was utilized with an irradiation energy of $7.37 \mathrm{~J} / \mathrm{cm}^{2}$ to ensure adequate adhesion of each layer. These parameters were used to fabricate the logo for the Organic Mechatronics \& Smart Materials Laboratory and a 3D cone. The 3D models of the structures can be seen in Figure 4.8(a) and Figure 4.8(d). The logo model consisted of 4 layers and the $1.5 \mathrm{~mm}$-tall cone consisted of 14 layers. Optical images of the fabricated 3D structures are shown in Figure 4.8.

The 3D cone illustrates the layered appearance characteristic of 3D printed structures. The 3D geometry can be more accurately represented by decreasing the layer 
thickness. Thinner photopolymer layers are better suited for fabricating fine features since they require a reduced dose of light energy for polymerization to propagate the full thickness of the layer. This reduced light-energy dose limits the amount of curing outside the irradiated area, leading to the optimal fabrication of fine features.

The fine features apparent in the printed logo illustrate how the minimum feature resolution affects the geometry of the produced part: small cavities, such as the threads of the light bulb, were filled while thin features, such as the polarity symbols on the magnet, were larger than desired. In general, sharp corners in the original geometry were rounded in the fabricated component. Despite the prescribed irradiation energy working well for the single layer feature resolution test, it resulted in the features of multilayer structures to become over-irradiated. This phenomenon is due to previous layers receiving additional irradiation as subsequent layers are cured. This effect is typically compensated for by adding a non-reactive light absorber to decrease the depth of light penetration (Choi et al., 2009).

\subsubsection{FTIR analysis}

Fourier-transform infrared spectroscopy (FTIR) of photopolymerized PPy, UDMA, and PPy/UDMA samples are depicted in Figure 4.9. The FTIR spectrum of the photopolymerized polymers was measured with a Bruker Tensor II. The absorbance bands present within the FTIR spectrum of PPy and UDMA are alike since the two polymers have similar types of bonds. However, the magnitude of the peaks differs due to the quantity of each bond. The FTIR spectrum of photopolymerized PPy exhibits several characteristic bands at $3440 \mathrm{~cm}^{-1}\left(\mathrm{~N}-\mathrm{H}\right.$ stretching), $1530 \mathrm{~cm}^{-1}$ ( $\mathrm{C}-\mathrm{C}$ and $\mathrm{C}=\mathrm{C}$ ring stretching), $1448 \mathrm{~cm}^{-1}$ (C-N stretching), $1297 \mathrm{~cm}^{-1}$ (C-H and C-N stretching), $1170 \mathrm{~cm}^{-1}$ (ring breathing vibration) and $1041 \mathrm{~cm}^{-1}$ (C-H in-plane and out-of-plane deformation) (Saravanan et al., 2006; Kasisomayajula et al., 2010; Bhat et al., 2001; Kasisomayajula et al., 2016; Rinaldi et al., 2005). The strong absorbance band at $1702 \mathrm{~cm}^{-1}$ corre- 


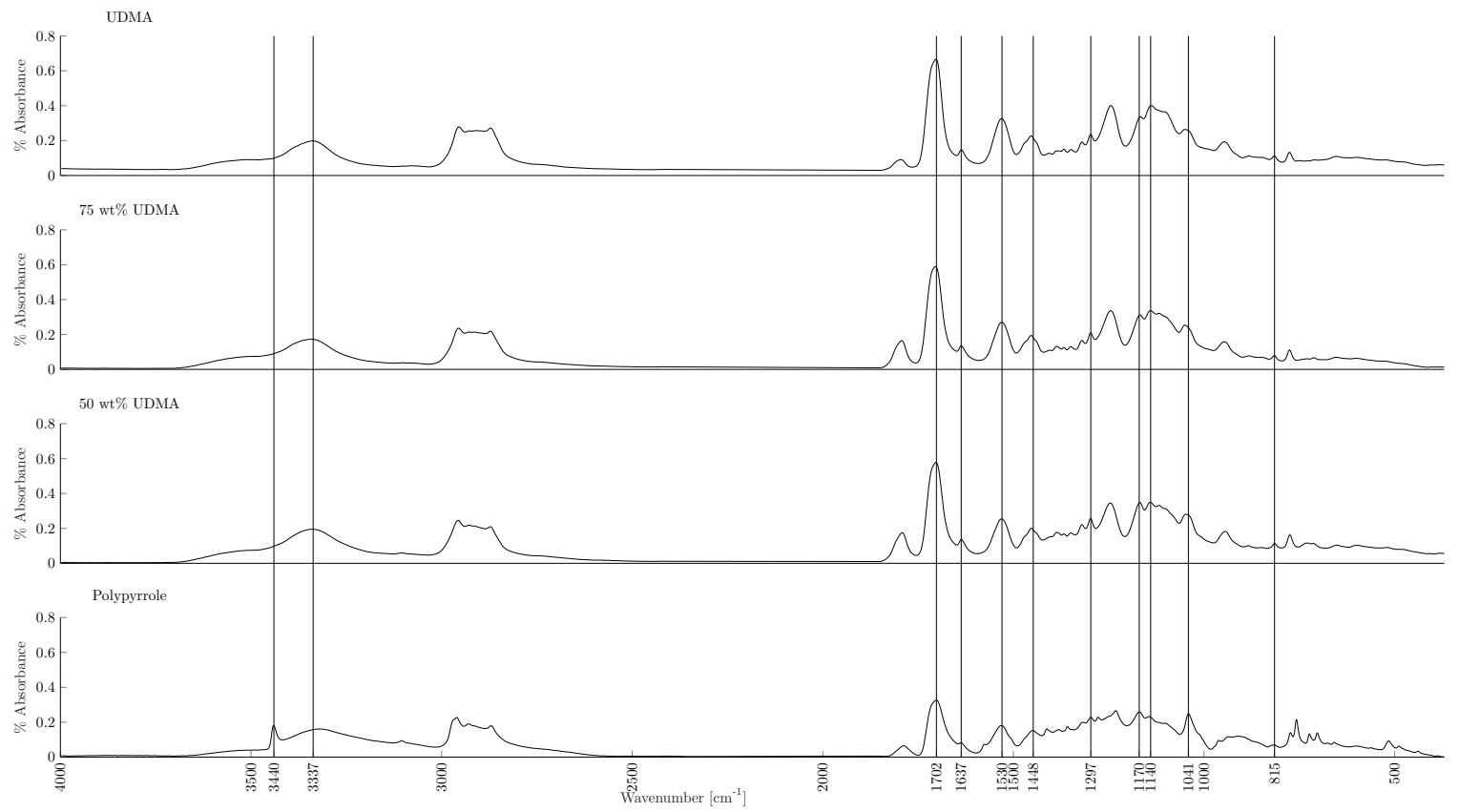

Figure 4.9: FTIR spectra of photopolymerized PPy, UDMA, and hybrid formulations containing $75 \mathrm{wt} \%$ UDMA. The presence of characteristic peaks of PPy and UDMA in the spectra of the hybrid polymer confirms the formation of both photopolymerized PPy and UDMA in the polymer.

sponds to the presence of a carbonyl group produced by the nucleophilic attack of water on pyrrole (Saravanan et al., 2006; Kasisomayajula et al., 2010).

The FTIR spectrum of UDMA has characteristic absorbance bands at $3337 \mathrm{~cm}^{-1}$ (-NH stretching), $1702 \mathrm{~cm}^{-1}$ (C=O stretching), $1637 \mathrm{~cm}^{-1}$ (C=C stretching) and $815 \mathrm{~cm}^{-1}$ ( $\mathrm{C}=\mathrm{C}$ twisting) (Kunwong et al., 2011; Atai et al., 2007). Hybrid photopolymer formulations containing a combination of the two polymers exhibit an FTIR spectrum that is a combination of the PPy and UDMA spectrums, therefore Figure 4.9 confirms the presence of both constituent polymers as anticipated.

\subsubsection{Cross-sectional morphology and composition analysis}

The cross-section of a four-layer hybrid photopolymer structure containing $75 \mathrm{wt} \%$ UDMA is shown in Figure 4.10. The cross-section of the polymer samples was prepared by first embedding the soft polymer in a UV curable embedding medium before 
Figure 4.10: SEM of the cross-section of a multi-layered hybrid photopolymer structure with $100 \mu \mathrm{m}$ thick layers shows no evidence of layering artifacts from the fabrication process but reveals a uniform dispersion of bright spots throughout the material.

cryo-fracturing under liquid nitrogen. The rough surface of the cross-section was a result of the cryo-fracturing process. There is no evidence of layering artifacts are present in the cross-section of the polymer arising from the layer by layer fabrication method however, there are bright spots present throughout the cross-section. Scanning electron microscopy (SEM) imaging was performed with a Hitachi S-3400N Microscope equipped with an Oxford Instruments INCA PentaFETx3 EDAX detector. Polymer samples were sputter coated with 60/40 Gold Palladium prior to imaging to minimize charging.

Energy dispersive X-ray spectroscopy (EDAX) elemental mapping was performed to identify the chemical composition of the bright spots observed in the cross-section. 


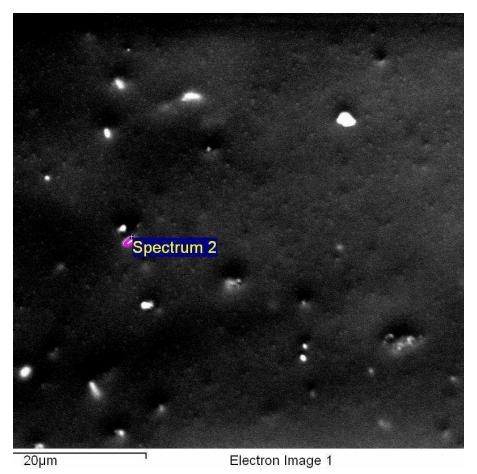

(a)

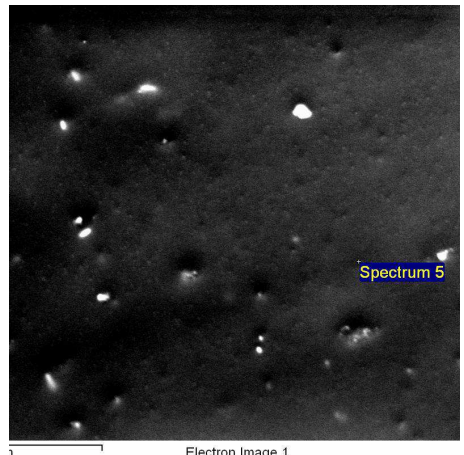

(c)

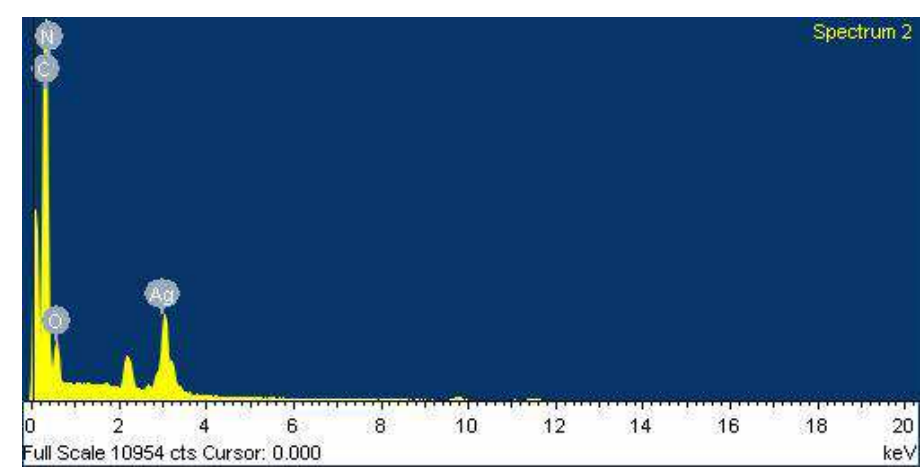

(b)

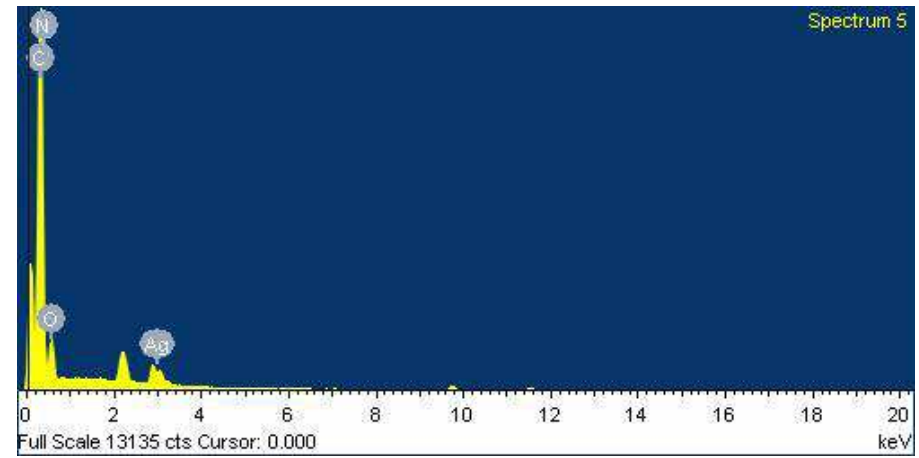

(d)

Figure 4.11: EDAX elemental mapping of bright spots in the cross-section of the photopolymer structure suggests the presence of highly conductive and well-dispersed Ag/PPy nanoparticles.

Figure 4.11(a) and 4.11(b) indicate the location and elemental composition of each site, and Figure 4.11(c) and 4.11(d) indicate the location and elemental composition of the parent phase. The elemental composition of each location is presented in Table 4.1. EDAX elemental mapping revealed that the bright spots in the cross-section of the polymer structure contain a relatively high concentration of silver. It is postulated that these regions likely consist of PPy and silver due to the interaction between PPy and silver which is known to form Ag/PPy core shell nanoparticles (Kasisomayajula et al., 2016; Yang and Lu, 2005; Zang et al., 2016; Nazar et al., 2015). 


\begin{tabular}{ccccc}
\hline & \multicolumn{4}{c}{ Composition [wt\%] } \\
\cline { 2 - 5 } Location & $\mathrm{C}$ & $\mathrm{N}$ & $\mathrm{O}$ & \multicolumn{1}{c}{$\mathrm{Ag}$} \\
\hline Spectrum 2 & 45.08 & 36.54 & 7.35 & 11.03 \\
Spectrum 5 & 52.99 & 38.88 & 7.62 & 0.51 \\
\hline
\end{tabular}

Table 4.1: Elemental composition of the hybrid photopolymer was ascertained via EDAX elemental mapping at the locations indicated in Figure 4.11. Spectrum 2 is of an Ag/PPy nanoparticle and Spectrum 5 is of the UDMA parent phase.

\subsubsection{Electrochemical activity}

Cyclic voltammetry (CV) was performed on the polymer samples to elucidate their electrochemical activity. The cyclic voltammetry experiment was performed with a computer controlled Keithley 2611 source meter. Cycling was performed between $0.8 \mathrm{~V}$ and $-0.2 \mathrm{~V}$ vs $\mathrm{Ag} / \mathrm{AgCl}$ reference electrode at a scan rate of $20 \mathrm{mV} / \mathrm{s}$ in an aqueous $\mathrm{NaNO}_{3}$ solution. Figure 4.12(a) depicts the cyclic voltammogram of PPy with respect to polymer samples containing UDMA, while Figure 4.12(b) isolates the data for samples containing the secondary UDMA polymer. The poor electrochemical activity observed for formulations containing UDMA is attributed to the high crosslink density of urethane acrylate polymers which greatly reduces the mobility of ionic species through the material. The network of urethane polymer surrounding the ionically conductive PPy regions effectively impedes ionic transport in the bulk. This reduced mobility results in all hybrid formulations containing UDMA exhibiting an ionic conductivity similar to that of pure UDMA.

\subsubsection{Electrical conductivity}

The electrical conductivity of the hybrid polymer formulation was measured with the 4-point probe technique. The 4-point probe technique is used because the result is not dependent on the electrode contact area (Blythe and Bloor, 2005). A Keithley 2611 source meter with a custom made 4-point probe stand, shown in Figure 4.13, was used 


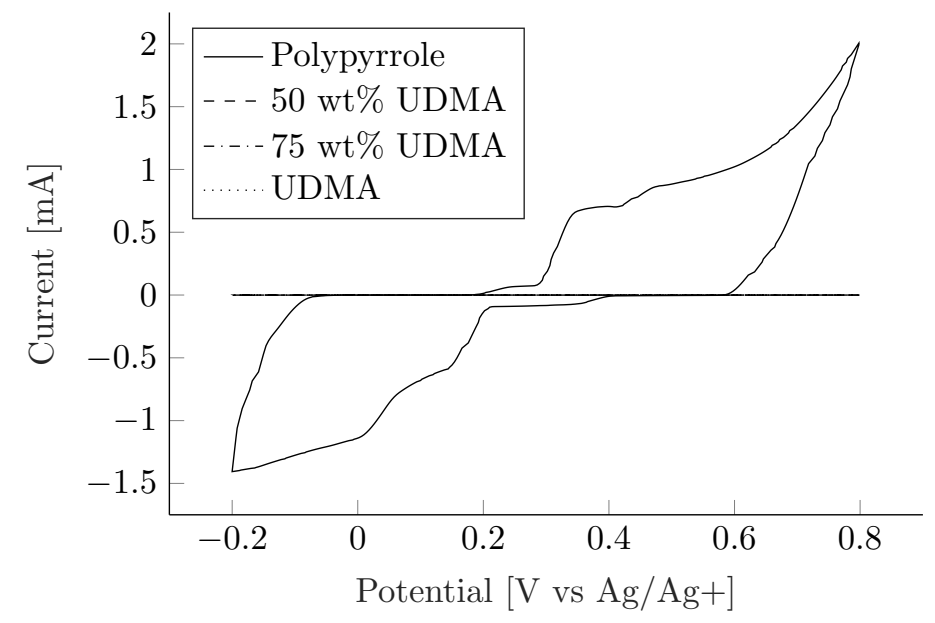

(a)

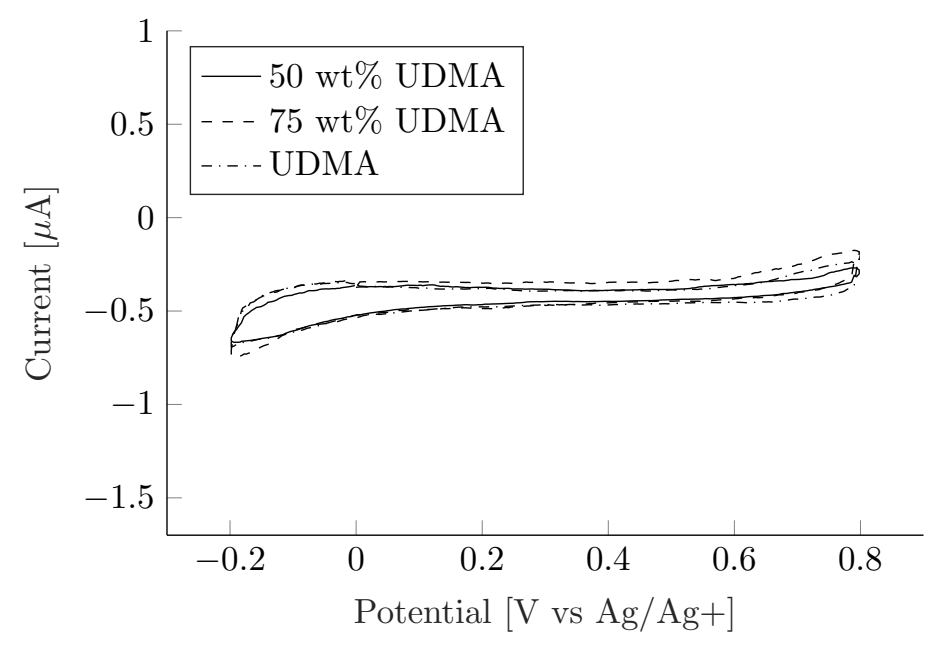

(b)

Figure 4.12: Cyclic voltammetry of (a) PPy compared to polymer samples containing UDMA and (b) samples containing various concentrations of UDMA, indicates the reduced ion mobility attributed to the highly crosslinked UDMA parent phase. 


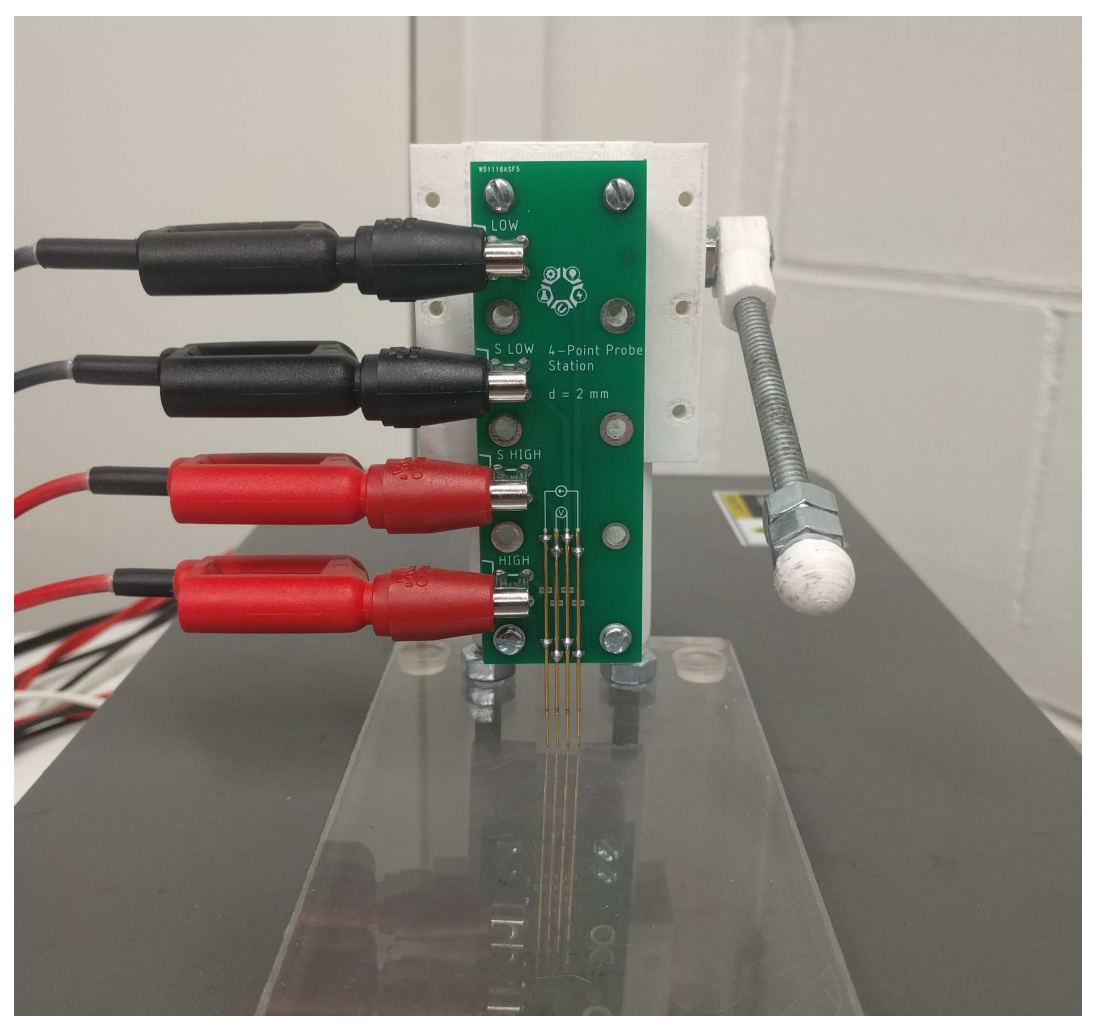

Figure 4.13: 4-point probe apparatus used to measure electrical conductivities.

to measure the electrical conductivity. The 4-point probe stand features a 3D printed rack and pinion stage and a circuit board with 4 spring loaded pins spaced $2 \mathrm{~mm}$ apart and connectors to the source meter. The apparent electrical conductivity of the polymer samples were calculated with the following formula:

$$
\sigma=\frac{l}{2 \pi V d}
$$

where $\sigma$ is the apparent conductivity, $I$ is the current passed through the sample, $V$ is the measured voltage and $d$ is the spacing between the probes. The calculated conductivity was not corrected for sample geometry due to an inability to accurately measure the sample thickness which will result in a lower conductivity than the true value.

Figure 4.14 indicates the relationship between the electrical conductivity of the poly- 
mer versus the concentration of the secondary UDMA polymer. The conductivity of the hybrid polymer exponentially decreased as the concentration of the urethane acrylate polymer increased. This trade-off is a common challenge for the development of hybrid PPy formulations. The electrical conductivity of hybrid photopolymer formulations reported in literature varies greatly based on the utilized secondary polymer and the composition of the formulation, for example: Martins et al. (2006) reported an electrical conductivity of $1 \cdot 10^{-3} \mathrm{~S} \cdot \mathrm{cm}^{-1}$ for a PET/PPy/Ag blend, Nazar et al. (2015) reported $6.8 \cdot 10^{-5} \mathrm{~S} \cdot \mathrm{cm}^{-1}$ for a PEGDA/PPy/Ag blend, Asmussen et al. (2015) reported $3.35 \cdot 10^{-5} \mathrm{~S} \cdot \mathrm{cm}^{-1}$ for a BisEMA/PPy blend and Rinaldi et al. (2005) reported $1 \cdot 10^{-6} \mathrm{~S} \cdot \mathrm{cm}^{-1}$ for PVC/PPy blend. Photopolymerized PPy/Ag films with no secondary polymer additive have been reported to have a conductivity of $0.51 \mathrm{~S} \cdot \mathrm{cm}^{-1}$ (Hodko et al., 2009). The aforementioned fabrication experiments found that a large concentration of the urethane acrylate polymer was required in the hybrid polymer formulation for it to be used effectively with the DLP 3D printing system. However, the electrical conductivity results demonstrate that the influence of the conjugated polymer properties decreases as the concentration of pyrrole decreases, illustrating that the designer is forced to balance process efficacy and electrical conductivity when this polymer formulation and fabrication technique are employed.

\subsubsection{Conclusions on pyrrole-UDMA hybrid polymer formulation}

The hybrid pyrrole-UDMA polymer formulation presented in this section exhibited a significant improvement in mechanical integrity, allowing it to be used with a free-surface vat polymerization additive manufacturing system to fabricate 3D structures. While this progress is an important milestone towards the fabrication of active 3D conjugated polymer devices, the current polymer has a number challenges that must be overcome to improve the performance of devices made from this hybrid polymer formulation.

While it is desired to have a flexible hybrid photopolymer, the pyrrole-UDMA for- 


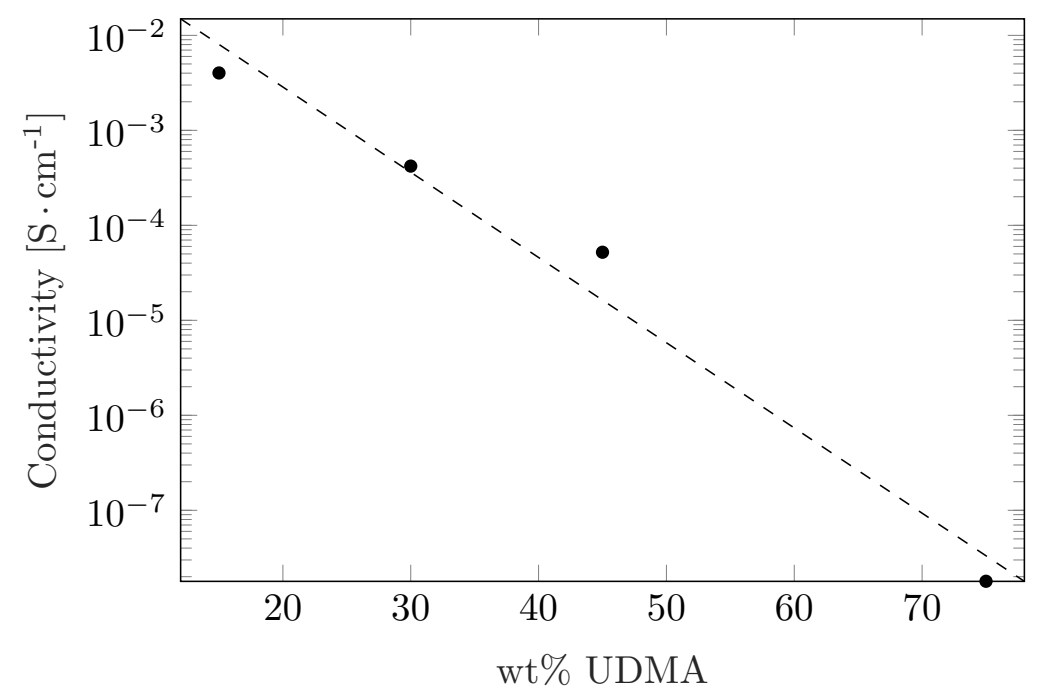

Figure 4.14: Electrical conductivity of the pyrrole-UDMA hybrid polymer versus the concentration of the secondary polymer shows an exponential decrease in conductivity as the concentration on the secondary polymer increases.

mulation exhibits mechanical properties similar to a gel which makes the produced structures delicate. Future formulations should investigate other secondary polymers that impart a greater mechanical integrity to the photopolymer formulation. The high concentration of the UDMA oligomer to form an overall polymer with sufficient mechanical integrity resulted in a low electrical and ionic conductivity, limiting the application of this polymer formulation. Alternative oligomers that have an improved ionic conductivity should be investigated so ions can reach the polypyrrole-silver islands within the secondary polymer matrix during electrochemical stimulation. Other additives such as light absorbers should be investigated to enable the fabrication of overhanging structures. 


\subsection{Photopolymerization of pyrrole-BEMA hybrid poly- mer formulation}

\subsubsection{Design of pyrrole-BEMA hybrid polymer formulation}

The primary insight gained from the pyrrole-UDMA photopolymer investigation was that the hybrid polymer formulation requires an ionically conductive secondary polymer that is compatible with polypyrrole. Ionically conductive polymers are commonly used in the area of solid polymer electrolytes for battery applications. A popular solid polymer electrolyte is Bisphenol A ethoxylate dimethacrylate (BEMA) which is a flexible polymer that is compatible with a wide range electrolyte solutions including LiTFSI which is commonly used for polypyrrole actuators. Due to the high viscosity of the BEMA oligomer, it is typically combined with poly(ethylene glycol) methyl ether methacrylate (PEGMA) as a reactive diluent to achieve the desired viscosity. The acrylate based polymers can be rapidly polymerized with a radical photoinitiator. Pyrrole-BEMA photopolymer formulations have been studied which form a solid polymer at a $41 \mathrm{wt} \%$ concentration of BEMA (ljeri et al., 2010). This oligomer is promising for the present formulation since a lower concentration of the secondary polymer is required which may allow the smart material properties of polypyrrole to have a much greater impact on the overall properties of the hybrid polymer.

In this section, BEMA was diluted with PEGMA at a weight ratio of 7:3. Fabrication tests with $20 \%$ increment concentrations of the BEMA and PEGMA solution found that a concentration then $40 \%$ could be employed without drastically impacting the mechanical integrity of the overall polymer. The same $8: 1$ molar ratio of pyrrole to silver was used. This photopolymer formulation was to be designed for used with the high resolution vat polymerization system discussed in Chapter 3 which uses a UV light engine. The radical photoinitiator 2,4,6-Trimethylbenzoyl-diphenyl-phosphineoxide (TPO) was 


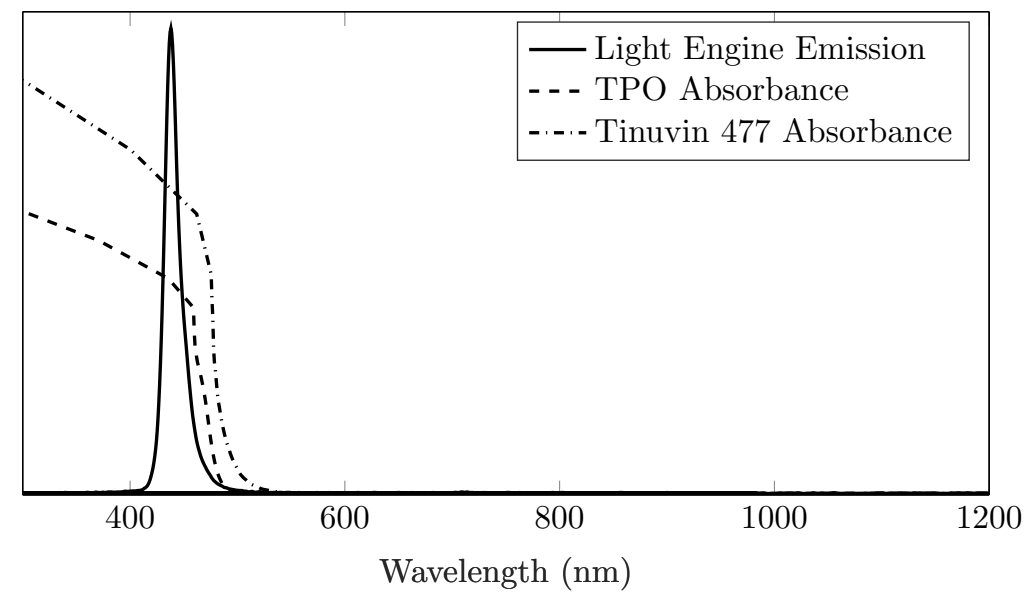

Figure 4.15: Emission spectrum of the light engine and absorption spectrum of the TPO photoinitiator and Tinuvin 477 UV absorber. The intensity and absorbance axes have been normalized.

added at a $5 \mathrm{wt} \%$ concentration to both photopolymerize BEMA and PEGMA and sensitize the silver ions to polymerize the pyrrole monomer. TPO has a strong absorbance overlap with the emission spectrum of the light engine as shown in Figure 4.15. To enable the fabrication of overhanging structures, the UV absorber Tinuvin 477 was incorporated into the photopolymer formulation at a concentration of $1 \mathrm{wt} \%$. The concentration of the photoinitiator and UV absorber in the polymer formulation was selected based on the manufacturer's recommendation. The absorbance spectrum of Tinuvin 477 can also be found in Figure 4.15. The emission spectrum of the light engine and absorption spectrum of the photoinitiator and UV absorber were measured with a BLACK-Comet UV-VIS spectrometer.

The pyrrole used in the pyrrole-BEMA hybrid formulation was distilled prior to use to improve the dissolution of silver nitrate in the monomer solution, eliminating the need for additional solvents. The pyrrole monomer is slightly unstable and will slowly polymerize in the absence of an initiating system. The solubility of silver nitrate decreases as the concentration of pyrrole oligomers increase in the monomer solution. Distilled pyrrole was stored in a fridge under a blanket of nitrogen to slow the rate of polymerization. 


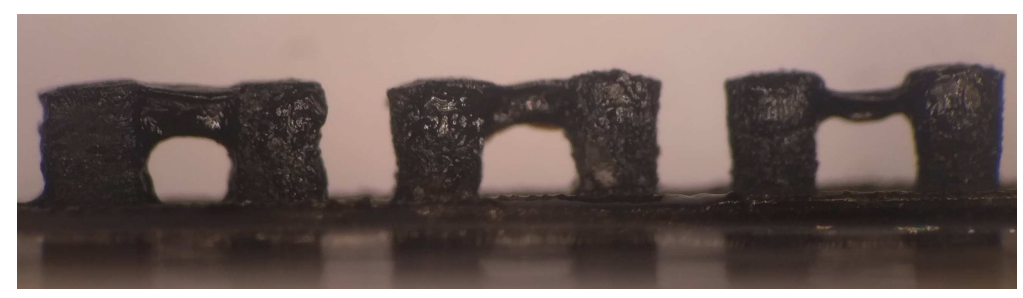

Figure 4.16: Bridge structure used to characterize the depth of cure of the pyrroleBEMA formulation. The overhanging layer was cured for a prescribed amount of time and the produced depth of cure was measured with an optical microscope.

\subsubsection{Depth of cure}

The reactivity of the pyrrole-BEMA photopolymer formulation was evaluated with the depth of cure experiment described in Chapter 2. The experiment was performed with a different method compared to the technique used to characterize the pyrrole-UDMA formulation. The depth of cure experiment was performed by fabricating the overhang structures shown in Figure 4.16 where the top overhang layer was cured for a prescribed amount of time. This methodology is advantageous since an array of overhang structures can be created in a single print job, greatly decreasing the time to perform the experiment. The thickness of the overhang structure was measured with an optical microscope. The working curve for the pyrrole-BEMA photopolymer is shown in Figure 4.17. The working curve of the photopolymer was used to select an appropriate irradiation time for a specified layer thickness when building multi-layered 3D structures.

\subsubsection{Electrochemical activity}

Cyclic voltammetry was performed on the hybrid pyrrole-BEMA polymer formulation to study the electrochemical activity. Polymer samples $9.6 \times 6.4 \times 0.5 \mathrm{~mm}$ in size were used for the experiment. The cyclic voltammetry experiment was performed with a similar method to that used for the pyrrole-UDMA polymer with a maximum potential of $+1.5 \mathrm{~V}$ and a minimum potential of $-1 \mathrm{~V}$ vs $\mathrm{Ag} / \mathrm{AgCl}$ reference electrode at a scan rate of $50 \mathrm{mV} / \mathrm{s}$ in an aqueous $\mathrm{NaNO}_{3}$ solution. Figure 4.18 depicts the cyclic voltammo- 


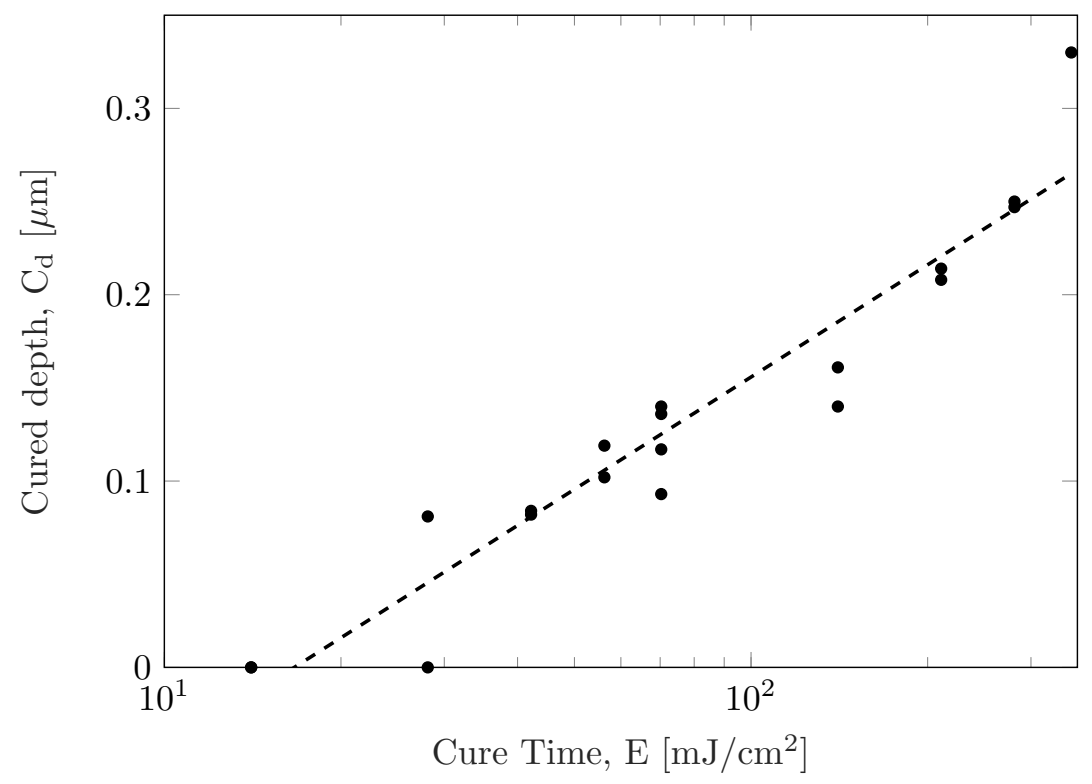

Figure 4.17: Depth of cure results for the pyrrole-BEMA polymer formulation confirms the hybrid polymer formulation agrees well with the depth of cure model described in Section 2.2.5.

gram of the hybrid PPy-BEMA polymer. The electroactivity of the PPy-BEMA polymer was greatly improved compared to the PPy-UDMA formulation. This result illustrates an important material property for the development of a hybrid polymer formulation since ionic conductivity is required for the development of conjugated polymer actuators. Chapter 5 will investigate whether the motion of ions will correspond to a volume change which can be utilized for actuation technologies.

\subsubsection{Electrical conductivity}

The electrical conductivity of the pyrrole-BEMA polymer was measured with the 4-point probe apparatus previously used for the PPy-UDMA hybrid polymer. The relationship between the concentration of BEMA-PEGMA in the photopolymer formulation and the resulting conductivity of the overall polymer is shown in Figure 4.19. As with the UDMA secondary polymer, the electrical conductivity exponentially decreases with increasing concentration of the secondary polymer. The electrical conductivity of the polymer 


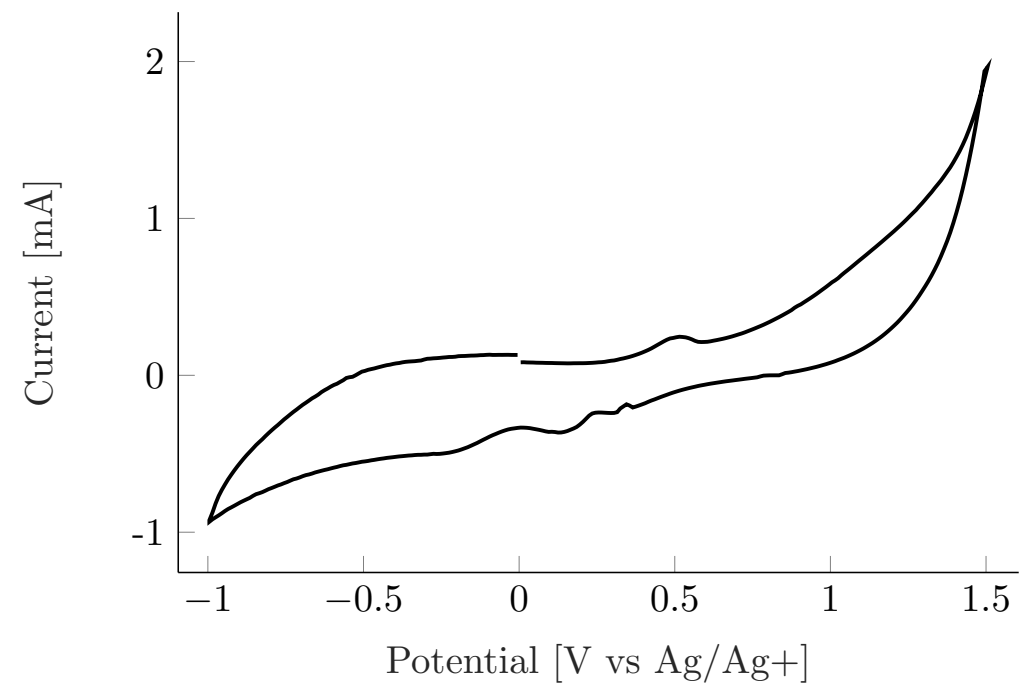

Figure 4.18: Cyclic voltammogram of the hybrid pyrrole-BEMA polymer formulation shows improved ionic conductivity compared to the pyrrole-UDMA formulation.

samples was similar to the values observed with the PPy-UDMA hybrid polymer.

\subsubsection{D structure fabrication}

The capability of the pyrrole-BEMA formulation to fabricate complex 3D structures was evaluated with the fabrication of a $2.5 \mathrm{~mm}$ tall standard benchmarking model shown in Figure 4.20. Desirable results were obtained with the following fabrication parameters: a layer thickness of $25 \mu \mathrm{m}, 30 \mathrm{~s}$ initial cure time to ensure adequate adhesion of the first layer to build plate, $1.5 \mathrm{~s}$ cure time for the following layers and a settling time of $30 \mathrm{~s}$. The cure time was selected based on the depth of cure experiment in Section 4.3.2 and the settling time was selected based on the settling time study in Section 3.3.4. The produced structures were rinsed with acetone and deionized water to remove uncured polymer from the fabricated part.

Overall, the pyrrole-BEMA formulation was able to fabricate the desired geometry of the standard benchmarking model. The addition of Tinuvin 477 allowed the overhangs present in the roof structure to be accurately produced while also limiting the amount of curing outside the irradiated areas. Fine features in the geometry such as the inner 


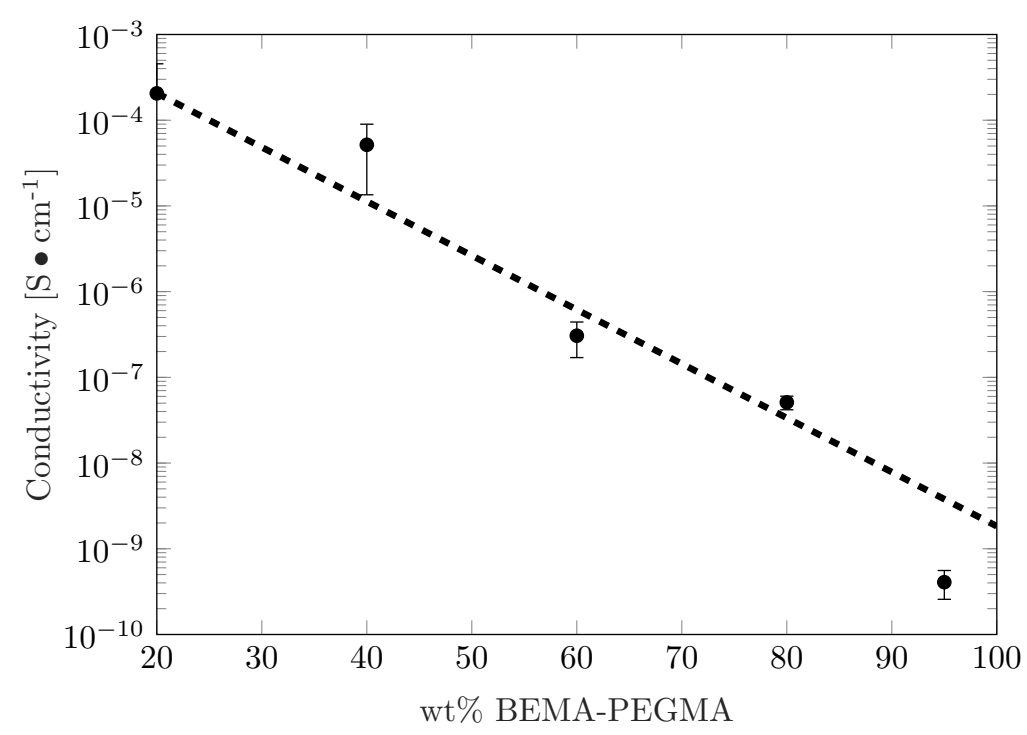

Figure 4.19: Electrical conductivity of the pyrrole-BEMA hybrid polymer versus the concentration of the secondary polymer shows an exponential decrease in conductivity as the concentration of the secondary polymer increases.

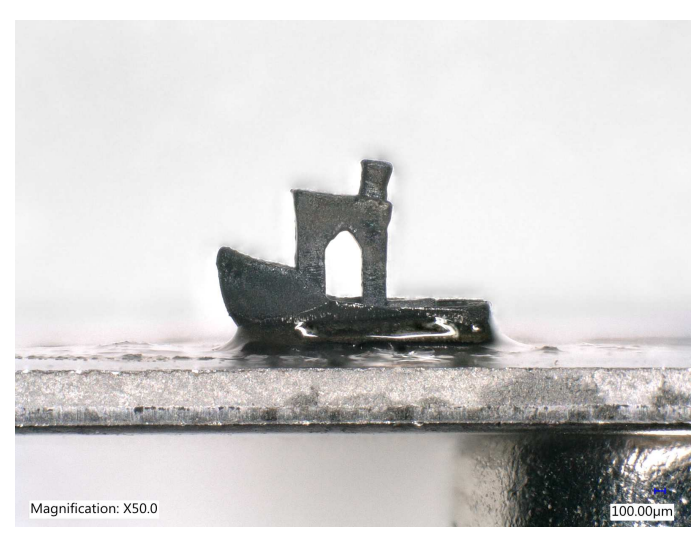

(a)

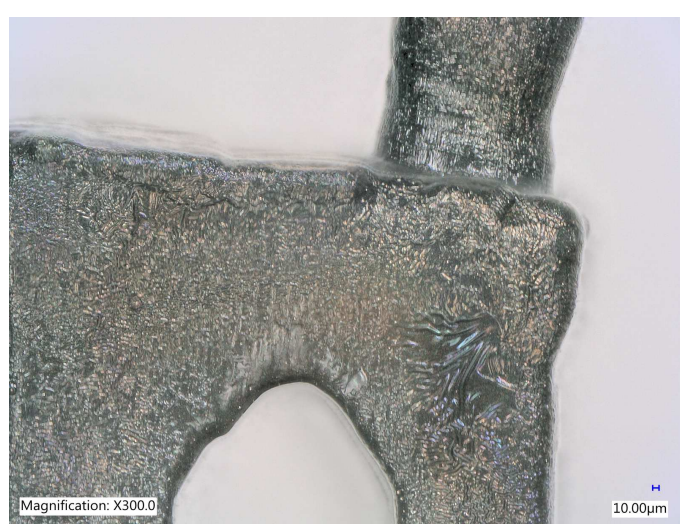

(b)

Figure 4.20: $2.5 \mathrm{~mm}$ tall standard benchmarking model with $25 \mu \mathrm{m}$ thick layers shows the ability for the designed pyrrole-BEMA hybrid polymer formulation to create complex microscale structures. 
hole of the chimney and the rear fishing rod holder are slightly overcured. Reduced irradiation times caused the pillars holding the roof to be too weak causing the roof of the boat to collapse, illustrating how the low stiffness of the pyrrole-BEMA photopolymer must be considered when designing 3D geometries with this material.

Due to the use of an acrylate based secondary polymer, the produced 3D structures exhibited a small amount of volume shrinkage. The volume shrinkage of the geometry was not observed while fabricating the structures but rather after the part had been washed and dried. As a result, the printed structure would commonly peel off the build plate. To compensate for the shrinkage effect, the produced part was adhered to the build surface with an adhesive glue after drying, which can be seen at the base of the printed structure in Figure 4.20.

\subsubsection{Multi-material structure fabrication}

The multi-material feature of the high resolution vat polymerization additive manufacturing system was applied to the fabrication of a prototype microfluidic channel with an embedded conjugated polymer pressure sensor. The device shown in Figure 4.21 was fabricated in three stages: first, the base of the device was made from a conventional photopolymer, then the conjugated polymer sensing element was built and finally the microfluidic channel was built around the sensing element.

The central vat in the multi-material system was filled with acetone to clean the photopolymer structures when switching between polymer vats. The build plate was dried with nitrogen gas after acetone washing. The device was designed to have a thin sensing element to minimize the number of washing procedures. The build plate was manually checked between material changes to ensure the structure was adequately cleaned. It was observed that agitation from moving the build surface in and out of the washing vat was insufficient to clean all of the liquid photopolymer and additional cleaning was required with an acetone wash bottle. Future development of the high 


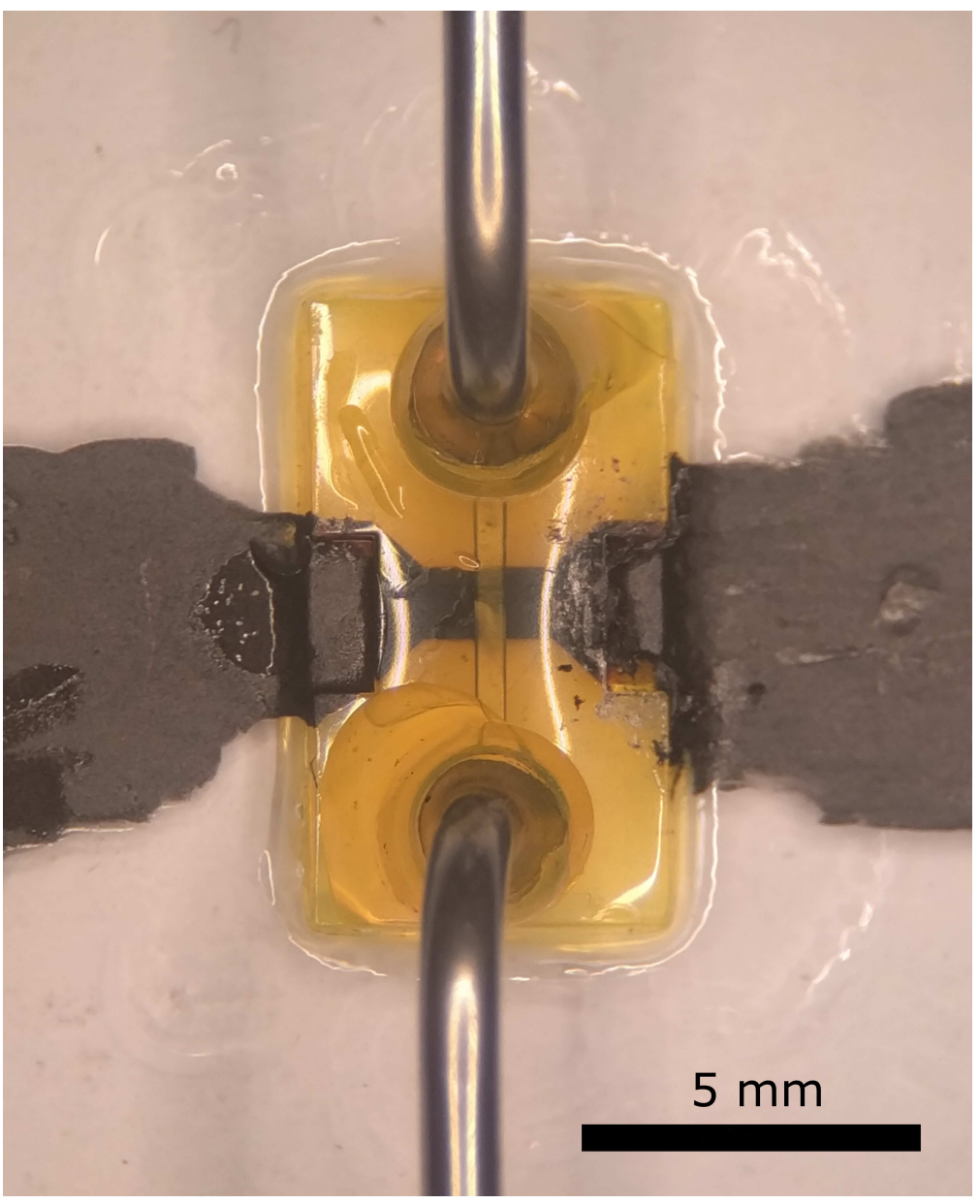

Figure 4.21: Multi-material 3D printed prototype microfluidic channel with an embedded conjugated polymer pressure sensor. 
resolution vat polymerization apparatus should investigate the use of an active washing station with a recirculating pump to clean the build surface.

The device was tested by connecting the inlet port to a Nordson EDF pneumatic fluid dispenser and measuring the change in the resistance of the sensing element with a Keithley 2611 source meter. Pneumatic connections were made to the microfluidic device by gluing right angle syringe needles in the inlet and outlet ports. The exposed terminals of the conjugated polymer sensing element were painted with a carbon based adhesive (PELCO ${ }^{\circledR}$ Conductive Carbon Glue, 16050) to improve the reliability of electrical connections.

The device was tested to inlet pressures of $50 \mathrm{psi}$, however, no significant change in the resistance of the sensing element was observed. It is anticipated that an improved performance would be observed if the sensing element was redesigned to experience a greater strain when fluid pressure is applied. Despite the lack of sensing response, this device showcases the capability of the fabrication system to produce multi-material devices with embedded sensing components.

\subsubsection{Conclusions on pyrrole-BEMA photopolymer formulation}

The hybrid polymer formulation developed in this section represents a significant advancement in the 3D printing conjugated polymer structures. The blend of BEMA and PEGMA as the secondary polymer exhibited an improved compatibility with polypyrrole to yield an overall polymer with enhanced mechanical integrity at a reduced secondary polymer concentration compared to the PPy-UDMA formulation. The enhanced mechanical integrity allowed more complex micro-scale 3D structures to be fabricated with the high resolution free-surface vat polymerization additive manufacturing system. The addition of the UV absorber Tinuvin 477 enabled the accurate fabrication of overhang structures and reduced curing outside the irradiated areas. The use of BEMA and PEGMA resulted in a significant increase in the ionic conductivity which is required 
for the fabrication of conjugated polymer actuators. Chapter 5 will investigate the application of the hybrid PPy-BEMA polymer to sensing and actuation technologies. The concentration of reagents in the presented polymer formulation are not optimal and were selected based on manufacturer recommendations, formulations presented in the literature, or preliminary concentration studies. Future studies are required to identify the affect of each component in the polymer formulation on the resulting material properties to identify the optimal concentration of each reagent.

\subsection{Photopolymerization of pyrrole-silver in commercial photopolymer formulations}

\subsubsection{Integration of pyrrole-silver in commercial photopolymer for- mulations}

With the success of incorporating of conventional photopolymers into the pyrrole-silver formulation, it was hypothesized that pyrrole and silver nitrate may be added to commercially available photopolymer to make them electrically conductive. The yellow photopolymer resin from B9Creations used in Chapter 3 was investigated for used as the secondary polymer. A solution containing an 8:1 molar ratio of pyrrole to silver nitrate was added in $20 \mathrm{wt} \%$ increments to the yellow photopolymer resin. Rectangular polymer samples, $9.6 \times 6.4 \times 0.5 \mathrm{~mm}$ in size, were cured for electrical conductivity measurement. The polymer formulations exhibited curing characteristics near that of the pure yellow photopolymer. As the concentration of the pyrrole-silver formulation increased, the photopolymer samples appeared to become more flexible compared to the stiff properties of the yellow photopolymer. The polymer formulation containing an $80 \mathrm{wt} \%$ concentration of the pyrrole-silver solution suffered from poor mechanical integrity. 


\subsubsection{Electrical conductivity}

The electrical conductivity of the hybrid polymer samples was measured with the 4point probe apparatus previously used to measure the PPy-UDMA hybrid polymer. The relationship between the concentration of secondary yellow photopolymer resin in the photopolymer formulation and the resulting conductivity of the overall polymer is shown in Figure 4.22. As with the previous hybrid polymer samples, the electrical conductivity exponentially decreased with increasing concentration of the secondary polymer. The electrical conductivity of the polymer samples was similar to the values observed with the PPy-UDMA and PPy-BEMA hybrid polymers.

The electrical conductivity of the hybrid polymer samples is near to that of photopolymer formulations that use a conductive filler. Mu et al. (2017) report an electrical conductivity of $2.7 \cdot 10^{-4} \mathrm{~S} \cdot \mathrm{cm}^{-1}$ for a commercial photopolymer containing $0.3 \mathrm{wt} \%$ carbon nanotubes (CNT) which is near the conductivity measured for hybrid polymer samples containing 80 wt\% of the pyrrole-silver nitrate solution. The pyrrole-silver nitrate solution presents an alternative additive to traditional conductive fillers. While a greater concentration of the pyrrole-silver nitrate is required compared to other conductive fillers, the conjugated polymer solution is advantageous since the pyrrole-silver nitrate solution is easily incorporated into other photopolymer resins without the use of special dispersing techniques. The pyrrole-silver nitrate solution is initially transparent which limits the effect on the curing characteristics of the host photopolymer resin.

\subsubsection{Conclusions on pyrrole-silver in commercial photopolymer formulations}

The investigation presented in this section showed that pyrrole and silver nitrate may be added to commercially available photopolymer resins to make them electrically conductive. The ability to use commercially available photopolymer resins is beneficial since 


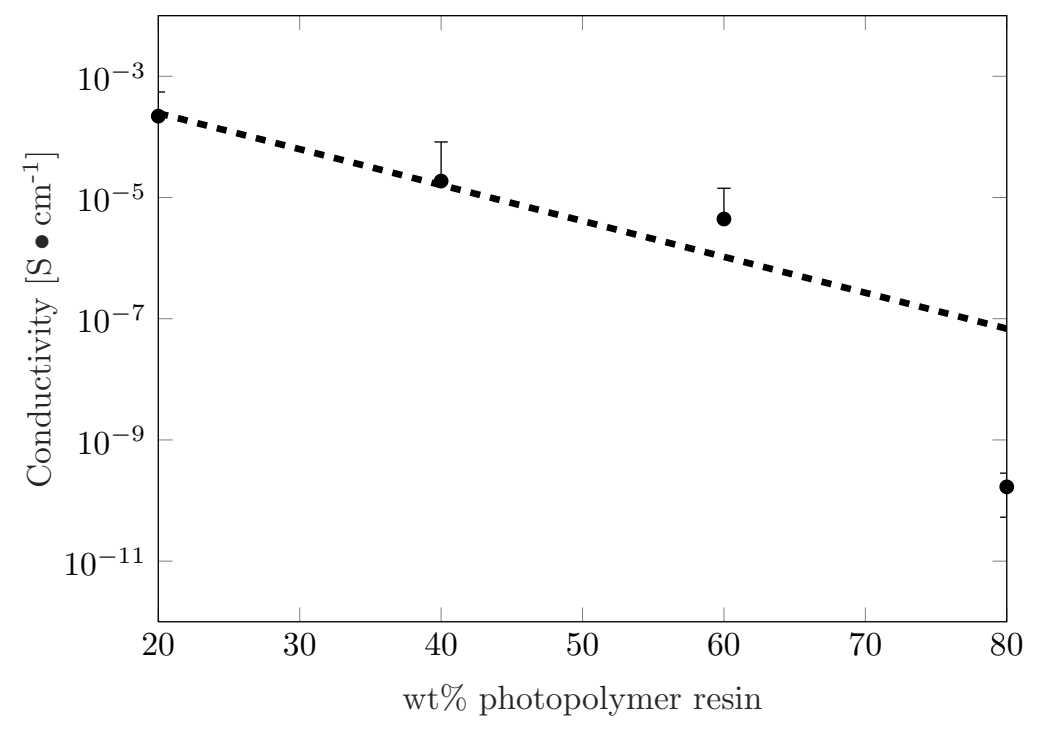

Figure 4.22: Electrical conductivity of the hybrid polymer of pyrrole and a conventional photopolymer versus the concentration of the secondary polymer shows an exponential decrease in conductivity as the concentration of the secondary polymer increases.

they have been fine tuned for desirable curing properties. The hybrid polymer formulation presents an alternative solution to traditional conductive fillers to form electrically conductive photopolymer resins. Future studies are required to understand the overall impact the pyrrole-silver nitrate additive has on the properties of the resulting polymer.

\subsection{Chapter summary}

This chapter presented the iterative design of a photosensitive polypyrrole formulation that is compatible with vat polymerization additive manufacturing for the fabrication of 3D conjugated polymer structures. After an existing photosensitive pyrrole-silver formation was found to be incompatible with vat polymerization additive manufacturing, hybrid polymer formulations were investigated which take on the smart material properties of PPy and the mechanical properties of a secondary polymer. A hybrid polymer formulation containing a BEMA oligomer was ultimately designed that is capable of being fabricated into complex 3D conjugated polymer structures with microscale features. 
The hybrid conjugated polymer formations developed in this chapter show promise for a new generation of 3D conjugated polymer sensing and actuation technologies.

\section{List of references}

Asmussen, S., Arenas, G. and Vallo, C. (2015), 'Photopolymerization of pyrrole/methacrylate mixtures using $\alpha$-cleavage type photoinitiators in combination with iodonium salt', Synthetic Metals 209, 304-312.

Atai, M., Ahmadi, M., Babanzadeh, S. and Watts, D. C. (2007), 'Synthesis, characterization, shrinkage and curing kinetics of a new low-shrinkage urethane dimethacrylate monomer for dental applications', Dental Materials 23(8), 1030-1041.

Bhat, N. V., Gadre, A. P. and Bambole, V. A. (2001), 'Structural, mechanical, and electrical properties of electropolymerized polypyrrole composite films', Journal of Applied Polymer Science 80(13), 2511-2517.

Bi, Y. and Neckers, D. C. (1994), 'A visible light initiating system for free radical promoted cationic polymerization', Macromolecules 27(14), 3683-3693.

Blythe, A. R. and Bloor, D. (2005), 'Electrical properties of polymers '.

Choi, J., Wicker, R. B., Cho, S., Ha, C. and Lee, S. (2009), 'Cure depth control for complex 3D microstructure fabrication in dynamic mask projection microstereolithography', Rapid Prototyping Journal 15(September 2008), 59-70.

Hodko, D., Gamboa-Aldeco, M. and Murphy, O. J. (2009), 'Photopolymerized silvercontaining conducting polymer films. Part I. An electronic conductivity and cyclic voltammetric investigation', Journal of Solid State Electrochemistry 13(7), 10631075. 
ljeri, V. S., Nair, J. R., Gerbaldi, C., Gonnelli, R. S., Bodoardo, S. and Bongiovanni, R. M. (2010), 'An elegant and facile single-step UV-curing approach to surface nanosilvering of polymer composites', Soft Matter 6(19), 4666-4668.

Kasisomayajula, S., Jadhav, N. and Gelling, V. J. (2016), 'Conductive polypyrrole and acrylate nanocomposite coatings: Mechanistic study on simultaneous photopolymerization', Progress in Organic Coatings 101, 440-454.

Kasisomayajula, S. V., Qi, X., Vetter, C., Croes, K., Pavlacky, D. and Gelling, V. J. (2010), 'A structural and morphological comparative study between chemically synthesized and photopolymerized poly(pyrrole)', Journal of Coatings Technology and Research 7(2), 145-158.

Kunwong, D., Sumanochitraporn, N. and Kaewpirom, S. (2011), 'Curing behavior of a UV-curable coating based on urethane acrylate oligomer: the influence of reactive monomers', Songklanakarin J. Sci. Technol 33(2), 201-207.

Marino, T. L., Martin, D. and Neckers, D. C. (1995), 'Chemistry and properties of novel fluorone visible light photoinitiators', Adhesives Age 38(4), 22.

Martins, C. R., De Almeida, Y. M., Do Nascimento, G. C. and De Azevedo, W. M. (2006), 'Metal nanoparticles incorporation during the photopolymerization of polypyrrole', Journal of Materials Science 41(22), 7413-7418.

Mu, Q., Wang, L., Dunn, C. K., Kuang, X., Duan, F., Zhang, Z., Qi, H. J. and Wang, T. (2017), 'Digital light processing 3D printing of conductive complex structures', Additive Manufacturing 18(November 2017), 74-83.

Nazar, R., Ronchetti, S., Roppolo, I., Sangermano, M. and Bongiovanni, R. M. (2015), 'In situ synthesis of polymer embedded silver nanoparticles via photopolymerization', Macromolecular Materials and Engineering 300(2), 226-233. 
Rinaldi, A., Kunita, M., Santos, M., Radovanovic, E., Rubira, A. and Girotto, E. (2005), 'Solid phase photopolymerization of pyrrole in poly(vinylchloride) matrix', European Polymer Journal 41(11), 2711-2717.

Saravanan, C., Shekhar, R. C. and Palaniappan, S. (2006), 'Synthesis of Polypyrrole Using Benzoyl Peroxide as a Novel Oxidizing Agent', Macromolecular Chemistry and Physics 207(3), 342-348.

Yang, X. and Lu, Y. (2005), 'Preparation of polypyrrole-coated silver nanoparticles by one-step UV-induced polymerization', Materials Letters 59(19-20), 2484-2487.

Yang, Y., Urbas, A., Gonzalez-Bonet, A., Sheridan, R. J., Seppala, J. E., Beers, K. L. and Sun, J. (2016), 'A composition-controlled cross-linking resin network through rapid visible-light photo-copolymerization', Polym. Chem. 7(31), 5023-5030.

Zang, L., Qiu, J., Yang, C. and Sakai, E. (2016), 'Preparation and application of conducting polymer/Ag/clay composite nanoparticles formed by in situ UV-induced dispersion polymerization', Scientific Reports 6(1), 20470. 


\section{Chapter 5}

\section{Application of 3D conjugated polymer}

\section{transducers}

This chapter investigates the capability for the PPy-BEMA hybrid polymer discussed in Chapter 4 to be used in sensing and actuation technologies. Electrochemical switching is used to stimulate the hybrid photopolymer and observe if the ion transfer is capable of inducing volumetric expansion and contraction for potential actuation applications. The compliant property of the hybrid polymer formulation was investigated for mechanical strain sensing applications. Strain to break and cyclic loading experiments were performed to study the change in the polymer structure's electrical conductivity in response to mechanical strain. The results from this chapter will provide insight into the types of devices that can be produced with the vat polymerization additive manufacturing system and hybrid conjugated polymer formulation. 


\subsection{Actuation performance of pyrrole-BEMA hybrid poly- mer formulation}

Conjugated polymers have been investigated for smart material actuators since this class of polymers is capable of ion driven volumetric expansion and contraction. The electromechanical transduction property of conjugated polymers is promising for microscale MEMS and lab on a chip devices since this material operates in a similar manner on both the macro- and micro-scale (Smela, 1999). The ability to fabricate 3D microscale conjugated structures is promising for the development of a new class of actuators that is capable of complex modes of actuation.

\subsubsection{Methods}

A thin hybrid polymer film, $8 \mathrm{~mm} \times 5 \mathrm{~mm}$ in size, containing 40\% BEMA-PEGMA secondary polymer was electrochemically stimulated with $\mathrm{a} \pm 2 \mathrm{~V}$ square wave with a frequency of $0.1 \mathrm{~Hz}$ in an electrolyte solution of $0.2 \mathrm{M}$ lithium bistrifluoromethanesulfonimidate (LiTFSI) in propylene carbonate shown schematically in Figure 5.1. The LiTFSI electrolyte is commonly used for polypyrrole actuators since the large relative size difference between the cation and anion of the lithium salt can induce a volume change upwards of 35\% (Spinks et al., 2009). The actuation experiment was preformed under an optical microscope to measure the volumetric expansion and contraction of the polymer. The tip displacement of the polymer film was analyzed with a custom MATLAB image processing script.

\subsubsection{Results and discussion}

Figure 5.2 shows the square wave excitation voltage signal and the resulting current flow through the apparatus. The tip displacement of the polymer film in response to the 


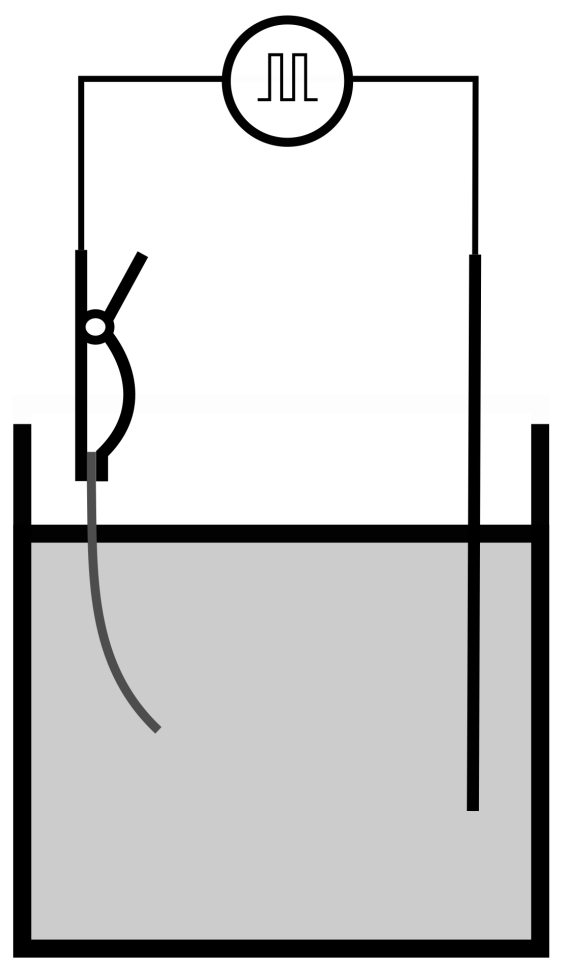

Figure 5.1: Electrochemical stimulation apparatus for measuring the actuation response of Pyrrole-BEMA hybrid polymer samples. The actuation experiment was preformed under an optical microscope to measure the volumetric expansion and contraction of the polymer. 


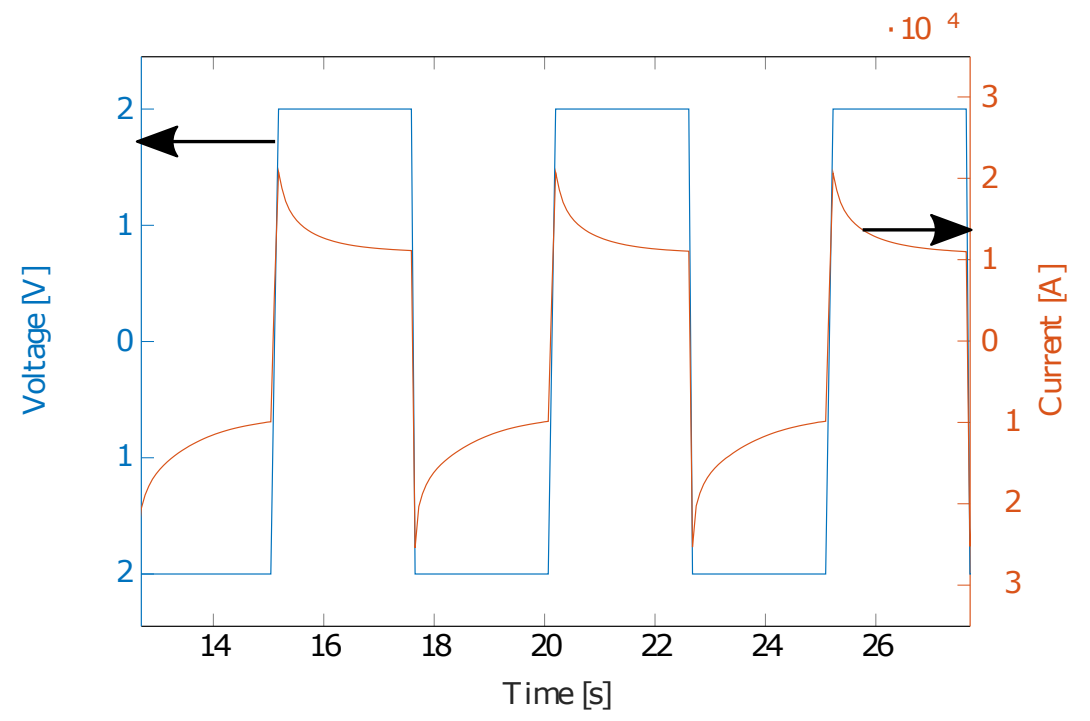

Figure 5.2: Electrochemical voltage excitation signal (blue) and resulting current flow (red) through the actuation test apparatus.

excitation voltage signal is shown in Figure 5.3. The tip displacement of the polymer film exhibits a step wise increase in displacement over time. The observed actuation response is likely due to the combination of solvent swelling, which contributes the linear increase in the tip displacement, and the volumetric expansion and contraction in response to the excitation signal, which results in the stepwise displacement response. Curling of the polymer film rather than uniform volume change in response to solvent swelling and electrochemical stimulation was likely due to stresses induced into the polymer film when it was peeled off the build plate. Greater tip deflections are expected to be observed if the hybrid polymer was combined with passive structures to amplify the electromechanical response of the polymer.

This experiment presents a preliminary investigation into the actuation response of the hybrid PPy-BEMA polymer to validate that the material is electromechanically active. A more detailed investigation into the actuation performance of the hybrid conjugated polymer is required to understand its capability within conjugated polymer devices. 


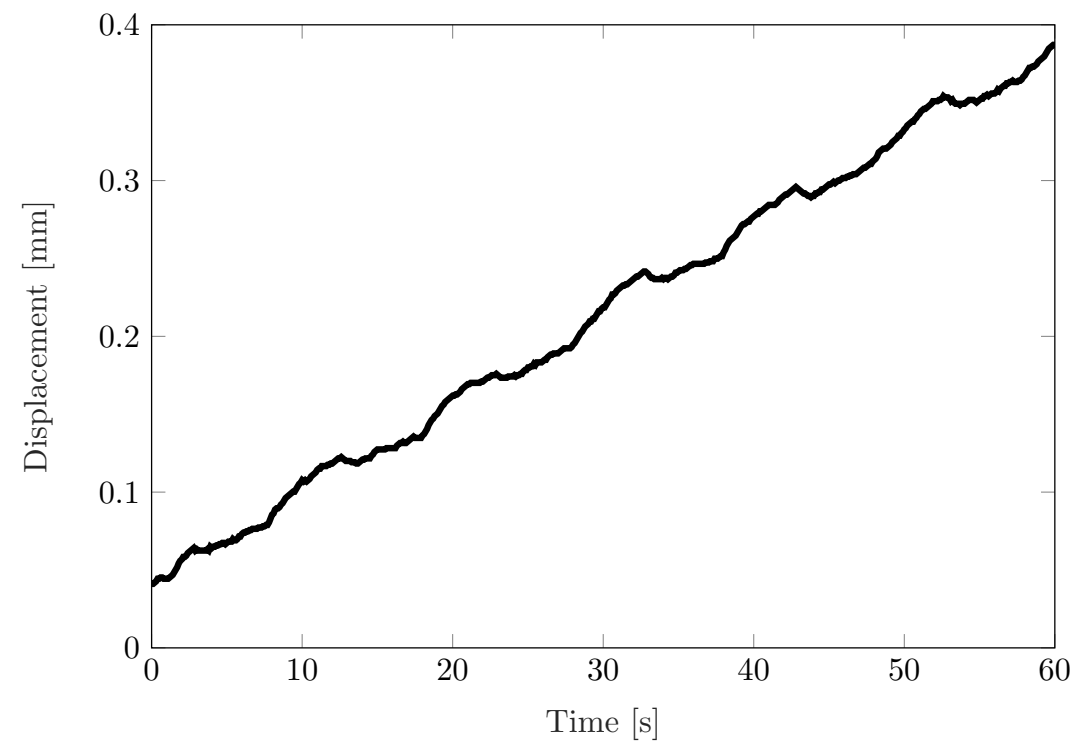

Figure 5.3: The tip displacement of an actuated polymer film in response to the excitation voltage signal shows a stepwise increase in displacement over time.

\subsection{Strain sensing performance of pyrrole-BEMA hybrid polymer formulation}

Conjugated polymers have been investigated for advanced sensing technologies due to a change in their electrical conductivity in response to external stimuli. Conducting materials with piezoresistive properties may be used as strain sensors to detect small motions. The change in electrical resistance when subjected to mechanical strain is characterized by the gauge factor $(G F)$ of the material which is defined as:

$$
G F=\frac{\Delta R / R_{0}}{\Delta l / I_{0}}=\frac{d \rho / \rho}{\epsilon_{l}}+1+2 v
$$

where $R_{0}$ is the unstrained resistance material with a length $I_{0}$. Under a mechanical deformation of $\Delta l$, the change in the material's resistance is defined as $\Delta R$. The change in resistance is contributed to by two factors, the change in the sample geometry $(1+2 v)$ and an intrinsic piezoresistive material property $\left(\frac{d \rho / \rho}{\epsilon_{l}}\right)$, where $\rho$ is resistivity, $\epsilon_{l}$ is strain and $v$ is the Poisson's ratio. 
This section aims to characterize the piezoresistive property of the hybrid conjugated polymer developed in Chapter 4 to evaluate its performance in strain sensing applications.

\subsubsection{Methods}

Tensile testing samples were fabricated in agreement with the ASTM D1708 standard (ASTM, 2014). The geometry of the tensile testing sample described in the ASTM D1708 standard is too large for the high resolution vat polymerization system to fabricate. The samples were prepared using a cast PDMS mold of the desired sample. The mold was filled with the polymer formulation and cured in a Spectroline XL 1500 UV crosslinker with $365 \mathrm{~nm}$ light for $30 \mathrm{~min}$. A hybrid polymer formulation containing BEMA as the secondary polymer at a concentration of $40 \mathrm{wt} \%$ was used for the piezoresistive experiments.

While the tensile testing samples were fabricated with a UV curing casting method rather than the additive manufacturing approach, this technique closely resembles a sample created with the additive manufacturing system where the profile of the tensile testing sample is aligned in the projected plane of the projector. If the sample was fabricated in an orientation such that the applied strain would be perpendicular to the layers in the polymer sample, it is expected that reduced mechanical properties would be observed due to stress concentrations at the interface between layers.

Piezoresistive testing was performed with a CellScale UniVert materials testing instrument equipped with a $10 \mathrm{~N}$ load cell. The resistance of the samples was measured using a Keithley 2611 source meter. The experimental setup for tensile testing is shown in Figure 5.4. For cyclic testing, samples were stretched to a maximum strain of $3 \%$ at a rate of $8 \mathrm{~mm} / \mathrm{min}$, held at the maximum stretched position for $1 \mathrm{~s}$, returned to its original length at a rate of $8 \mathrm{~mm} / \mathrm{min}$, and held at the relaxed position for $1 \mathrm{~s}$. This displacement protocol was repeated for 200 cycles, and resistivity measurements were 


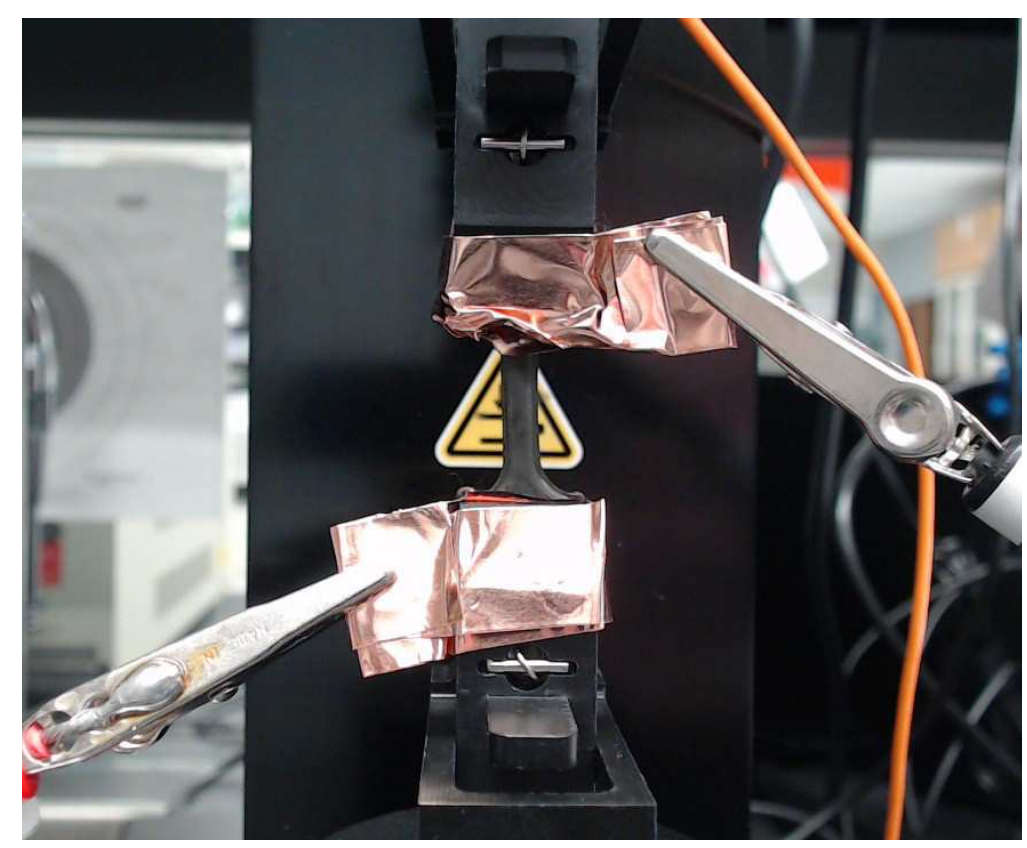

Figure 5.4: Pyrrole-UDMA hybrid polymer sample installed in a CellScale UniVert materials testing instrument for piezoresistive experiments. Copper tape on the grips of the tensile testing instrument was used to establish electrical connection to the polymer sample.

obtained at a rate of $5 \mathrm{~Hz}$. Post-cyclic testing, the samples were further displaced in tension at a rate of $3 \mathrm{~mm} / \mathrm{min}$ to failure.

\subsubsection{Cyclic strain response}

The typical response of the polymer sample under the cyclic tensile loading is shown in Figure 5.5. The amount of strain is indicated by the red line and the resistance of the polymer sample is shown by the blue line. As the strain on the polymer increased, the measured resistance of the polymer sample also increased.

The normalized resistance at the stretched and relaxed states over 200 cycles is shown in Figure 5.6, which illustrates a gradual decay in the nominal resistance, indicated by the decay of the resistance in the relaxed state. There is also a decrease in the piezoresistive response when subject to the $3 \%$ strain, indicated by a decreasing distance between the relaxed and stretched resistance over time. The decay in 


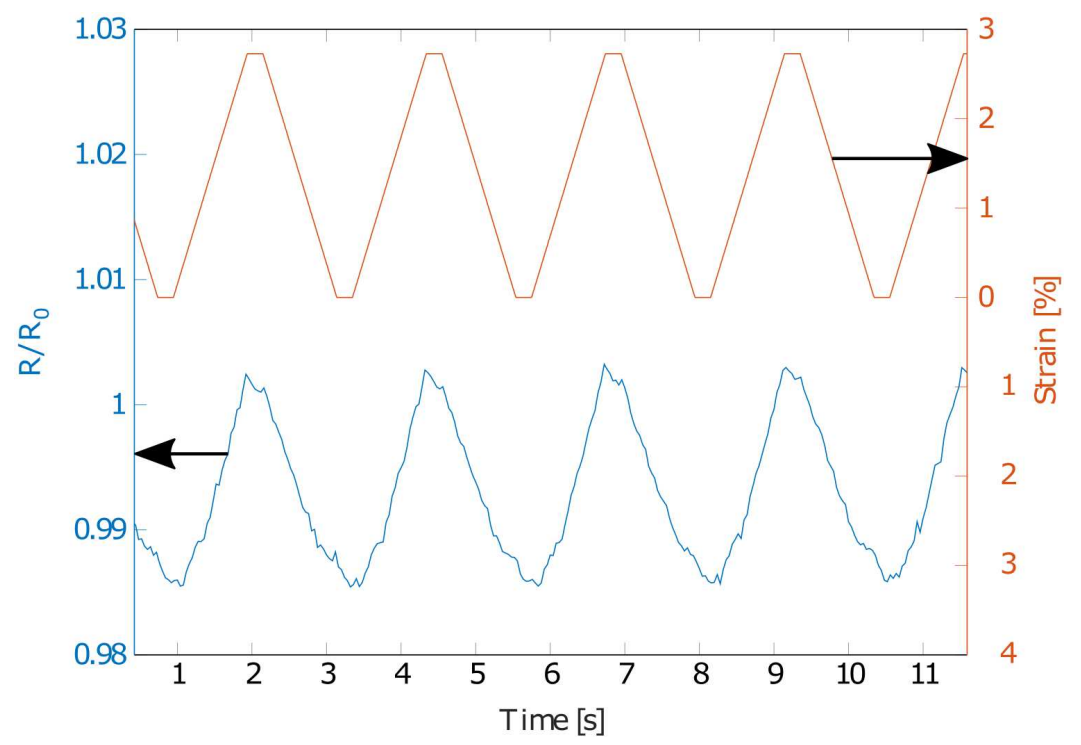

Figure 5.5: Plot of the excitation strain profile and the resulting change in resistance response of the hybrid conjugated polymer sample.

the piezoresistive response is clearly shown in Figure 5.7 with a plot of the gauge factor over the 200 loading cycles. The gauge factor of the material linearly decays with increased cycling.

The range of gauge factors measured with the hybrid conjugated polymer samples is similar to the work performed by Mu et al. (2017) who observed a gauge factor of 0.37 for polymer based strain sensors containing carbon nanotubes.

\subsubsection{Strain to break response}

Figure 5.8 shows a typical strain to break experiment with a plot of normalized resistance versus strain. The resistance of the tensile testing sample exponentially increased with strain. Based on 6 samples, the average strain to failure was $13 \%$, illustrating the compliant nature of the hybrid polymer formulation. 


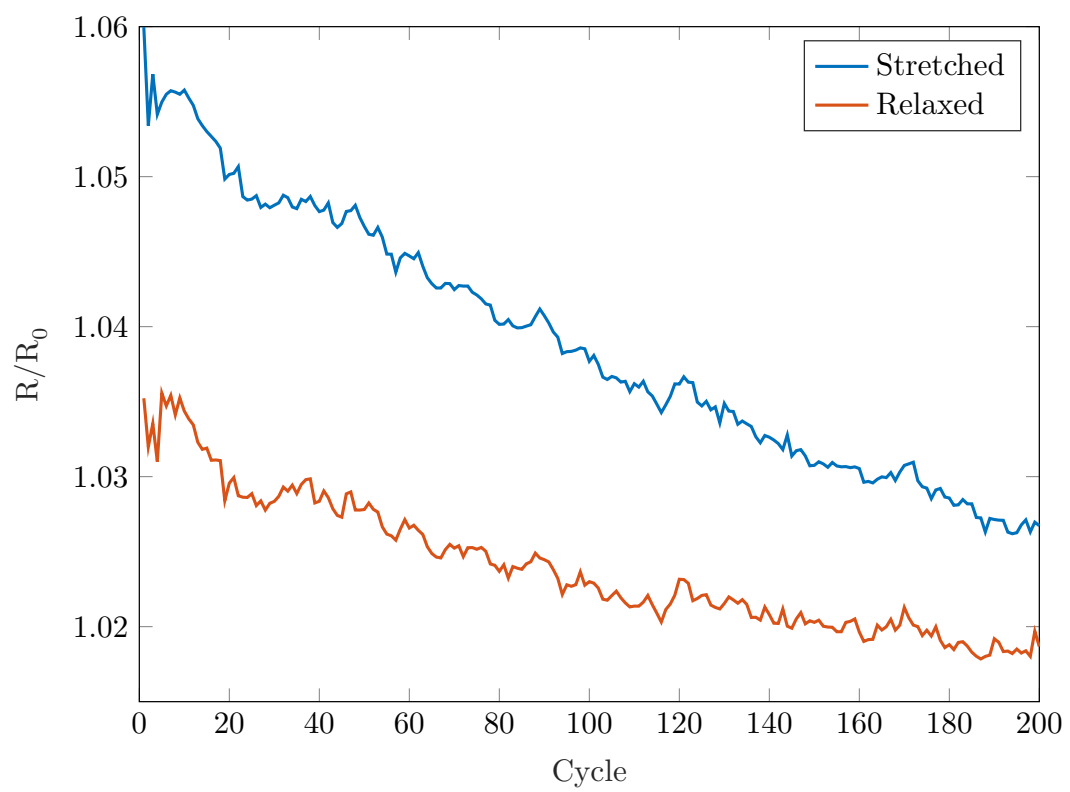

Figure 5.6: Normalized resistance of the hybrid polymer sample in the stretched and relaxed states over 200 loading cycles.

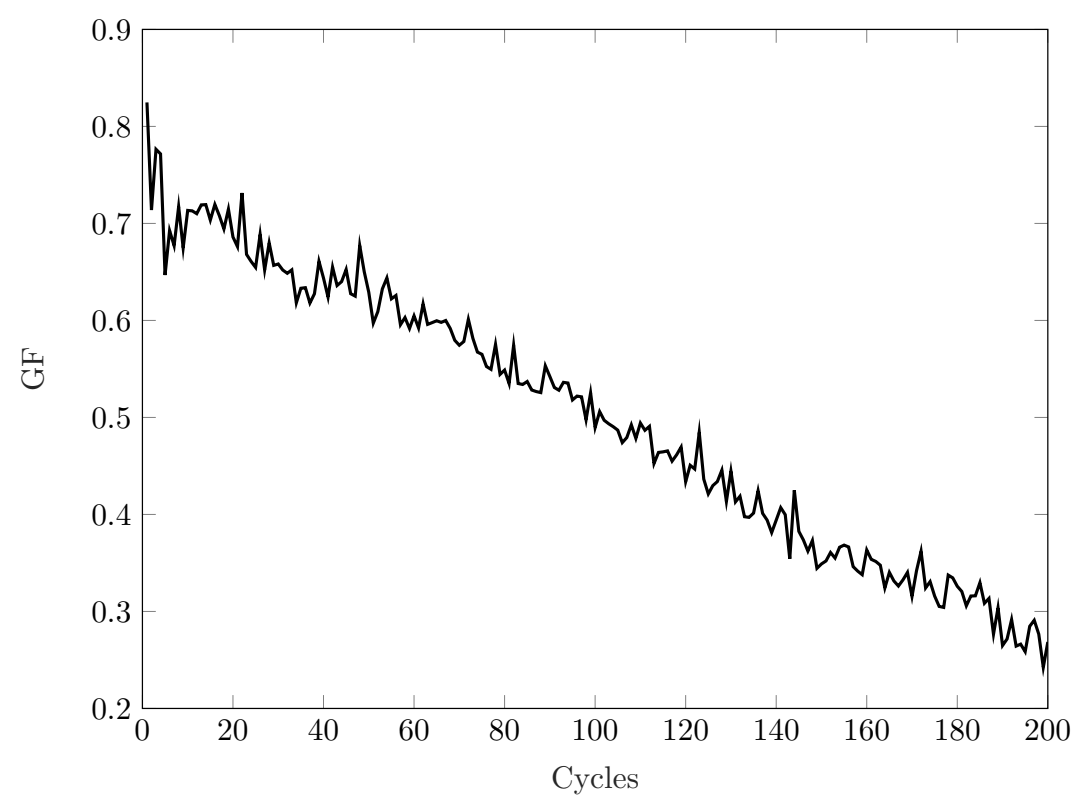

Figure 5.7: The gauge factor of the hybrid polymer sample over 200 loading cycles shows a linear decrease in the gauge factor with increased cycling. 


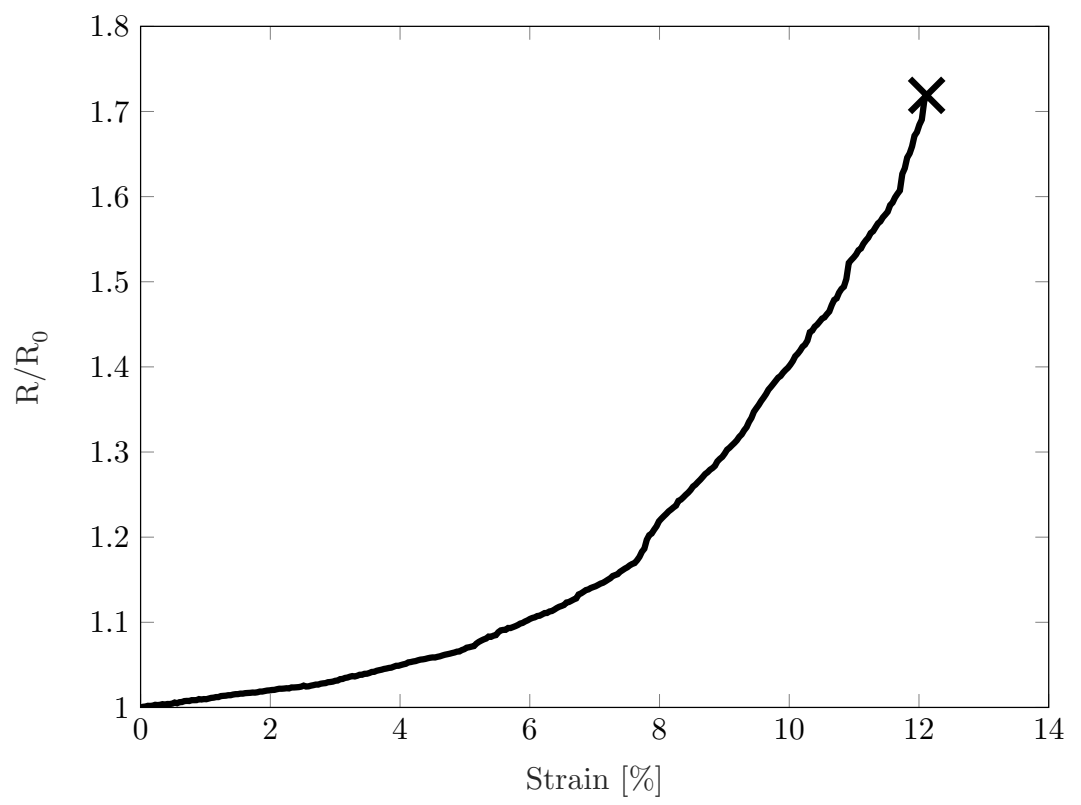

Figure 5.8: Typical strain to break response for hybrid polymer samples with a plot of normalized resistance versus strain.

\subsubsection{Discussion}

Based on the definition of gauge factor in Equation 5.1, for small gauge factors, the negative change in resistance due to the intrinsic piezoresistive property will compete against the positive change in resistance due to the change in geometry of the sample. The intrinsic and geometric piezoresistive components has a similar magnitude but opposite sign resulting in the small gauge factor.

It is hypothesized that when the polymer sample is strained, the reduction in crosssectional area causes the conductive polymer chains to come closer together resulting in a localized decrease in resistance but the change in resistance due to the change in geometry dominates over the length of the sample, causing the overall resistance of the polymer sample to increase. With prolonged cycling, the conductive polymer chains preferentially orient in the direction of strain resulting in both a decrease in the nominal resistance of the polymer sample and a concomitant decrease in the gauge factor of the material. 
Additional studies are required to further understand the piezoresistive property of the hybrid conjugated polymer structure. Improved gauge factors may be observed for compressive based strain sensors since both the intrinsic piezoresistive property and the change in geometry will be decreasing the sample resistance rather than competing against each other.

\subsection{Chapter summary}

This chapter investigated the mechanical strain sensing and actuation performance of the pyrrole-BEMA hybrid polymer discussed in Chapter 4. A stepwise increase in tip displacement of a polymer film was observed when electrochemically stimulated in a LiTFSI solution. The observed actuation response is likely due to the combination of solvent swelling and volumetric expansion and contraction in response to the excitation signal. Additional investigations are required to understand the actuation capability of the hybrid polymer formulation. The piezoresistive properties of the hybrid polymer were characterized with cyclic loading and strain to break experiments. The hybrid polymer exhibits an exponential increase in resistance when subjected to mechanical strain with an initial gauge factor of 0.75 . The gauge factor of the polymer sensor linearly decays with cycling, likely due to merging of the conducive islands in the hybrid polymer structure. The presented study is an important look at the transduction properties of the designed hybrid polymer formulation to inform the future design of devices with microscale 3D conjugated polymer structures. Future studies are required to optimize the hybrid polymer formulation to yield improved transduction properties. 


\section{List of references}

ASTM (2014), 'Standard Test Method for Tensile Properties of Plastics by Use of Microtensile Specimens', American Society for Testing and Materials 08(October), 5-9.

Mu, Q., Wang, L., Dunn, C. K., Kuang, X., Duan, F., Zhang, Z., Qi, H. J. and Wang, T. (2017), 'Digital light processing 3D printing of conductive complex structures', Additive Manufacturing 18(November 2017), 74-83.

Smela, E. (1999), 'Microfabrication of PPy microactuators and other conjugated polymer devices', Journal of Micromechanics and Microengineering 9(1), 1-18.

Spinks, G. M., Alici, G., McGovern, S., Xi, B. and Wallace, G. G. (2009), Conjugated Polymer Actuators: Fundamentals, in 'Biomedical Applications of Electroactive Polymer Actuators', John Wiley \& Sons, Ltd., West Sussex, United Kingdom, pp. 193227. 


\section{Chapter 6}

\section{Concluding Remarks}

\subsection{Summary of conclusions}

This thesis explored the issues surrounding the fabrication of 3D conjugated polymer structures using the vat polymerization additive manufacturing technique. The knowledge ascertained through these activities has prompted the following conclusions:

1. Conventional photosensitive polypyrrole formulations cannot be directly utilized with additive manufacturing technology. Conventional photosensitive polypyrrole formulations create a polymer particulate upon irradiation which does not retain its shape when used with additive manufacturing systems. The combination of a photosensitive polypyrrole formulation with traditional photopolymers to create a hybrid polymer formulation is required to form a solid polymer upon irradiation.

2. The vat polymerization additive manufacturing technique is a viable approach to fabricate microscale 3D conjugated polymer structures. The low stiffness of the hybrid conjugated polymer formulations requires the use of the free-surface vat polymerization technique to eliminate failure of the produced structure during the peeling step present in the fixed-surface technique. Precision equipment including a high pixel resolution light engine and wiper based laminating system may 
be used to produce 3D conjugated polymer structures with micro-scale features. Multi-material fabrication is a valuable feature to create microscale devices with embedded conjugated polymer structures.

3. Hybrid polypyrrole formulations containing BEMA as the secondary polymer, PEGMA as a reactive diluent, Tinuvin 477 as an absorber and TPO and silver nitrate as the photoinitiating system possess desirable curing properties for the fabrication of micro-scale structures while maintaining the electrical and ionic conductivity properties of polypyrrole.

4. Finally, hybrid polypyrrole formulations containing BEMA as the secondary polymer exhibit piezoresistive and electroactive properties which may be used in sensing and actuation technologies. Cyclic loading and strain to break experiments reveal that hybrid conjugated polymer structures exhibit an exponential increase in resistance when subjected to mechanical strain with an initial gauge factor of 0.75 that linearly decays with cycling. Electrochemical excitation of the hybrid polymer in a LiTFSI solution resulted in a stepwise increase tip displacement response illustrating the potential for this polymer to be unused in actuation technologies.

\subsection{Summary of contributions}

The most significant research contributions presented in this thesis are summarized as follows:

- The design of a multi-material vat polymerization fabrication system. A novel multi-material vat polymerization additive manufacturing system was designed to fabricate 3D conjugated polymers devices. The apparatus is capable of producing features with a $5 \mu \mathrm{m}$ pixel resolution. Multi-material capability enables the 
production of microscale devices with embedded conjugated polymer structures.

- A hybrid polypyrrole formulation that is compatible with the vat polymerization additive manufacturing technique. A hybrid photopolymer formulation was designed for the production of 3D conjugated polymer structures with light based additive manufactured technology. The use of BEMA as the secondary polymer enabled the production of complex microscale structures while maintaining the smart material properties of polypyrrole.

- Strain sensing performance characterization of hybrid polypyrrole structures. A preliminary investigation into the piezoresistive properties of hybrid polypyrrole structures was performed to provide insight into the types of devices that may be produced with the vat polymerization additive manufacturing system and hybrid conjugated polymer formulation.

\subsection{Recommendations for future research}

The vat polymerization additive manufacturing system and photosensitive hybrid conjugated polymer formulation will serve as a platform for the development of microscale 3D conjugated polymer transducers. The ability to fabricate 3D conjugated polymer structures will open up new transducer technologies that take advantage of unique 3D geometry. This technology is valuable for many applications in the fields of MEMS, microfluidics, and biomedicine to fabricate novel devices.

The present thesis was constrained to investigating photosensitive pyrrole formulations which used silver nitrate with a radical photoinitiator as the photoinitiating system but several other photoinitiating systems were discussed in Chapter 2. A broad study into the photopolymerization of pyrrole may be valuable to identify an optimal photoinitiating system. 
One of the most interesting properties of conjugated polymers is their ability to be used in actuation technologies. A brief investigation into the actuation performance of the conjugated polymer revealed a small amount of volumetric expansion and contraction in response to electrochemical stimulation. Additional investigations are required to understand the actuation capability of the hybrid polymer formulation and to study the response of $3 \mathrm{D}$ conjugated polymer actuators.

The investigation into the piezoresistive properties of hybrid pyrrole-BEMA structures revealed a complex response. An in-depth study into the piezoresistive properties hybrid pyrrole-BEMA structures should be performed consisting of both compression and tension testing at varied rates and amplitudes to inform the future design of devices featuring conjugated polymer strain sensors.

Finally, the addition of a pyrrole and silver nitrate to commercially available photopolymer formulations was found to be a promising alternative to traditional conductive fillers. Future studies should further investigate the overall impact the pyrrole-silver nitrate additive has on the properties of the resulting polymer. 


\section{Appendices}




\section{Appendix A}

\section{Prototype fixed-surface vat polymerization design}

This appendix will describe the design of the prototype fixed-surface vat polymerization additive manufacturing system. This technique is commonly used in commercial vat polymerization additive manufacturing systems since it provides precise control over the cured layer thickness with the use of a linear stage, therefore the fixed-surface vat polymerization design is a natural starting point for the instigation into vat polymerization of conjugated polymers.

The commercially available B9Creator 3D printer was used as a starting point for the prototype system. The B9Creator 3D printer, shown in Figure A.1, utilizes a fixed surface vat polymerization technique. The slide peel release mechanism is used in this system to release each layer from a PDMS window. While the slide peel release technique is effective in reducing the peeling forces on the printed part, it requires a much larger polymer vat which is unfavorable for photosensitive conjugated polymer structures since the reagents in the formulation are expensive and spoil over several hours. The polymer vat and build plate system shown in Figure A.2 was designed to utilize the B9Creator with a reduced volume of polymer.

A simple straight pull peel mechanism was employed in the modified build plate to minimize the volume of polymer required. The polymer vat was made from glass by gluing a $35 \mathrm{~mm}$ inner diameter glass tube to a $38 \mathrm{~mm}$ diameter glass circle with 5-minute epoxy. The 5-minute epoxy was found to be compatible with various available solvents including acetone, isopropyl alcohol, and propylene carbonate. The bottom of the polymer vat was coated with either cast PDMS or FEP film to reduce peeling forces.

The polymer vat was held in place between two metal plates. The bottom plate was fixed to the B9Creator and had a $33 \mathrm{~mm}$ hole in the center for projected light to pass up into the polymer vat. The top plate had 4 holes for bolts to secure the polymer vat along with a $34 \mathrm{~mm}$ hole for the build surface to pass through. The polymer vat was aligned between the holes in the top and bottom plates with a laser cut acrylic jig.

A cylindrical aluminum rod was utilized as a build plate. The aluminum rod had a diameter of $30 \mathrm{~mm}$, leaving a $2.5 \mathrm{~mm}$ clearance between the sides of the polymer vat for the photopolymer to be displaced. The cylindrical build plate was held to a steel plate with three embedded neodymium magnets. Using magnets to attach the build 


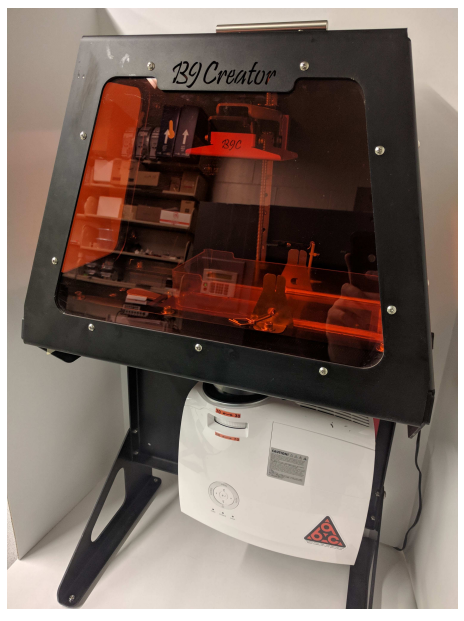

(a)

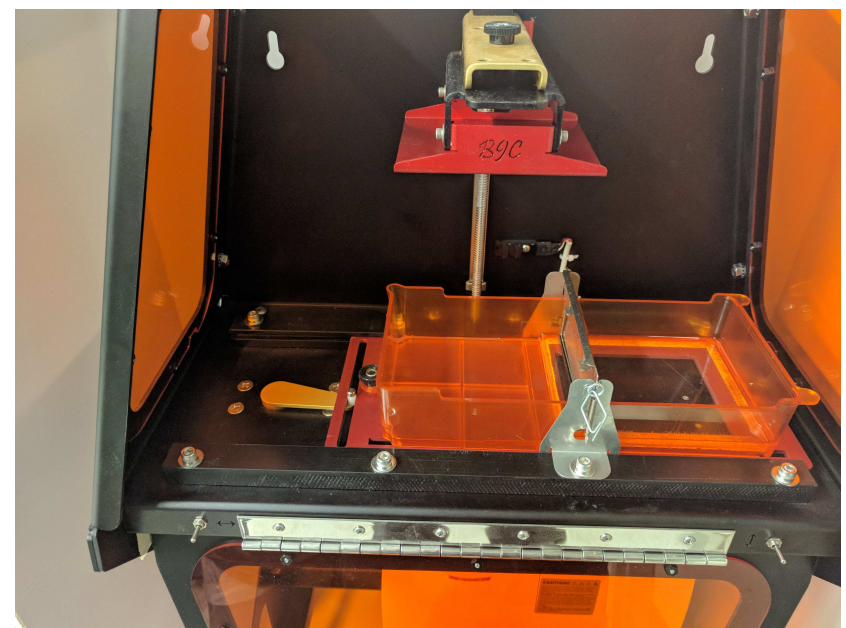

(b)

Figure A.1: Unmodified B9Creator 3D printer used in the preliminary study of vat polymerization of conjugated polymers.

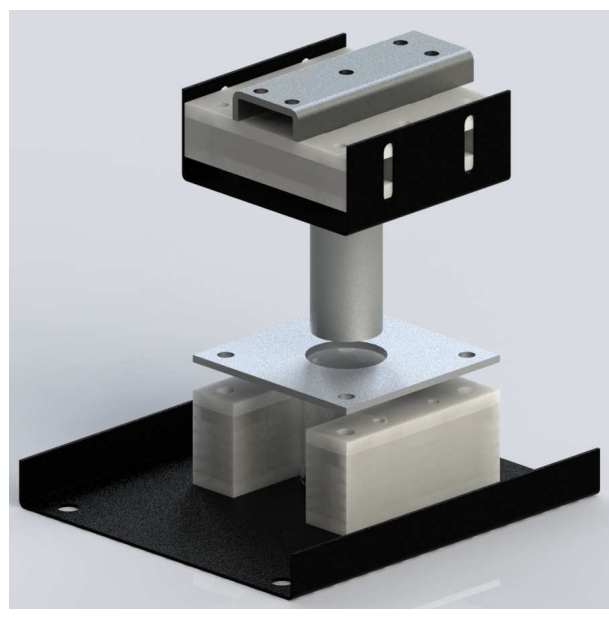

(a)

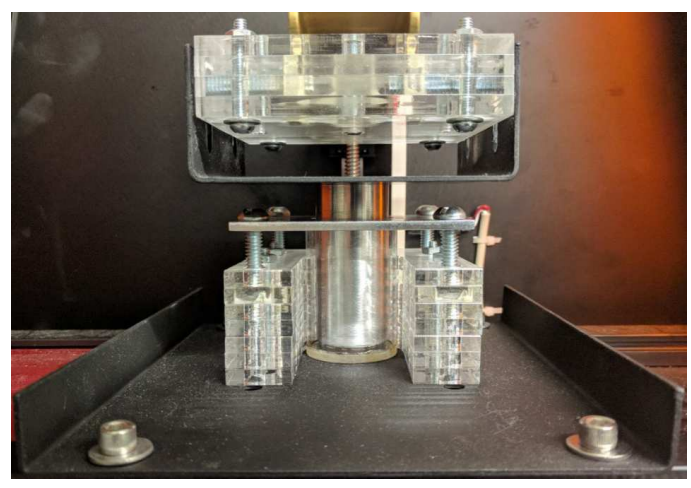

(b)

Figure A.2: Modifications made to the B9Creator 3D printer to reduce the build volume of the polymer vat. 
surface made for easy alignment with the polymer vat and removal of the produced structure. Since the existing build surface was non-magnetic, a custom fixture was designed that featured a magnetic steel surface. The steel surface was designed in a "U" shape with slots cut into the vertical surfaces so the orientation could be adjusted. The steel surface was bolted to a laser cut acrylic mounting block. Acrylic mounting block had locating holes in the top surface interface with the existing jig attached to the vertical build plate stage.

Since the new build surface extended significantly lower then the previous build surface, the software would move the build surface too low during the build plate height calibration procedure. To compensate for the height difference, a 3D printed extension to the build surface stage calibration photo-switch interrupter plate was installed which has a length equal to the height difference between the new and existing build surfaces. The interrupter plate extension made the software think the build surface was lower than reality, allowing the modified build surface to be used with the existing software. 


\section{Appendix B}

\section{Prototype free-surface vat polymerization design}

This appendix will describe the design of the prototype free-surface vat polymerization additive manufacturing system. This technique was investigated after concluding that the strength of the photosensitive polypyrrole formulation was not high enough to withstand the peeling step present in the fixed-surface vat polymerization technique. The free-surface technique overcomes the peeling step by curing the thin polymer layers on top of the previously cured layer, making it ideal for fabricating structures with low strength photopolymers.

A prototype free-surface vat polymerization additive manufacturing system was constructed by modifying a commercially available B9Creator 3D printer. The prototype system is shown in Figure B.1 and schematically in Figure B.2.

The existing DLP projector was oriented downward to project the images of each layer from above. A glass polymer vat was mounted in a 3D printed fixture directly below the DLP projector. The liquid level within the vat was controlled with a computerized syringe pump. A 4 in stainless steel needle bent in the middle at $90^{\circ}$ was held within the polymer vat by the 3D printed vat fixture to dispense the retract the photopolymer from the syringe pump. Ultra-Chemical-Resistant Versilon PVC Tubing was used between the syringe pump and the needle to prevent degradation from the photopolymer. The polymer vat was contained within an airtight chamber with a window in the top. Oxygen inhibition at the curing layer was mitigated by purging the chamber with nitrogen gas. A removable stainless steel sheet in the bottom of the polymer vat was used as a build surface.

As a result of the significant modifications to the B9Creator, the standard software and hardware for this printer were no longer compatible. An A4988 stepper motor driver and Arduino microcontroller were build on a breadboard to dispense or retract photopolymer from the syringe pump.

A Raspberry Pi 2 running NanoDLP, a program to host and control SLA and DLP 3D printers, was used to coordinate the additive manufacturing process. This software is very configurable and allowed custom commands to be communicated to the Arduino microcontroller after each layer was cured. To overcome surface tension forces between the previously cured layer and the photopolymer resin, the vat was flooded until 


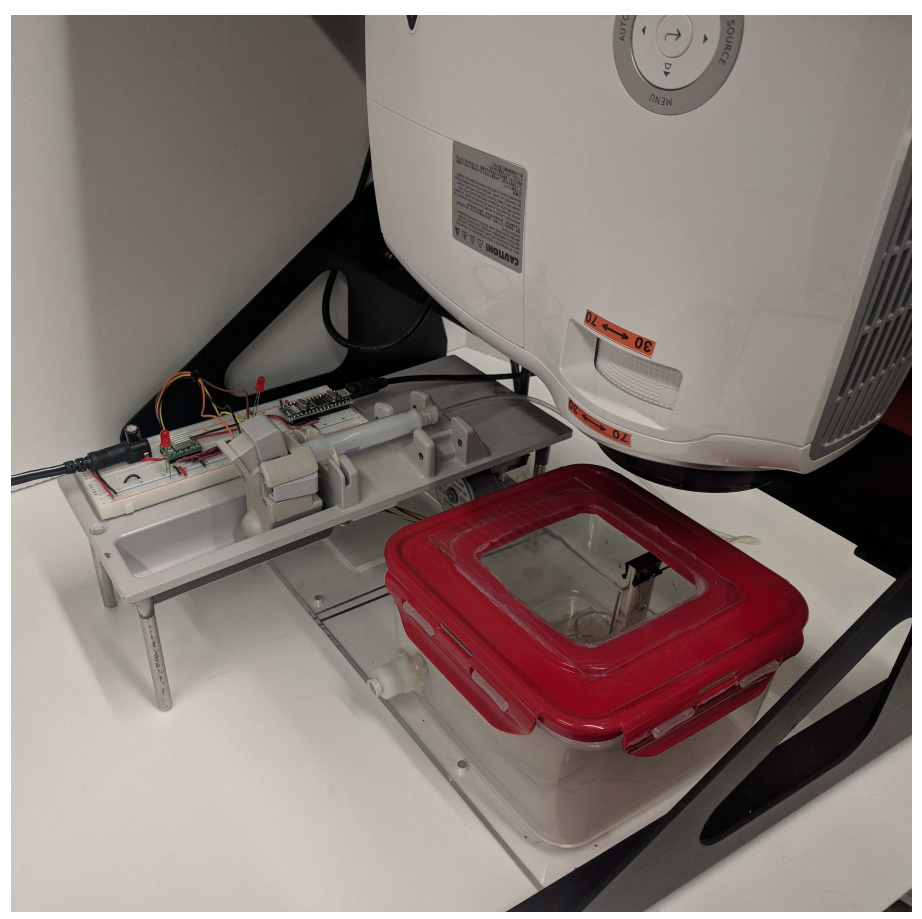

(a)

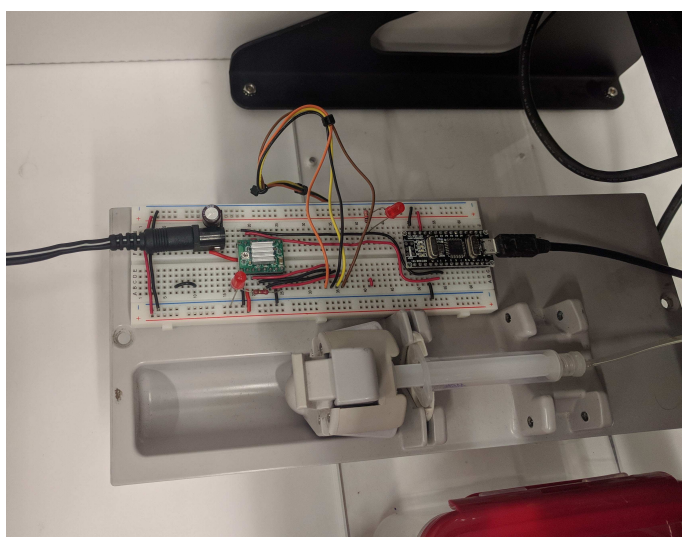

(b)

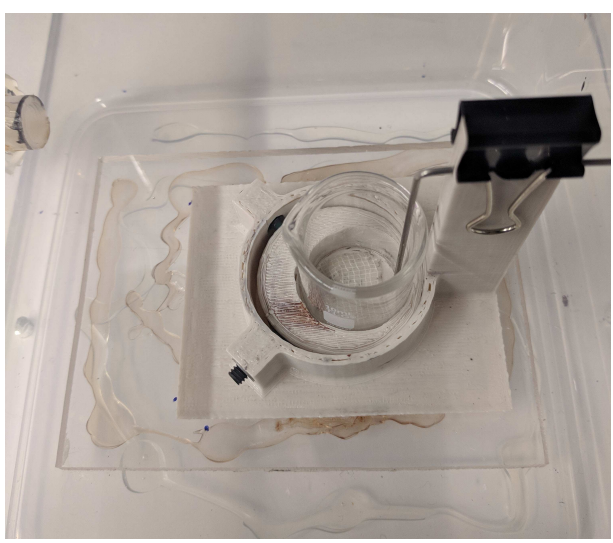

(c)

Figure B.1: Modifications made to the B9Creator 3D printer to print with the free-surface vat polymerization technique. 


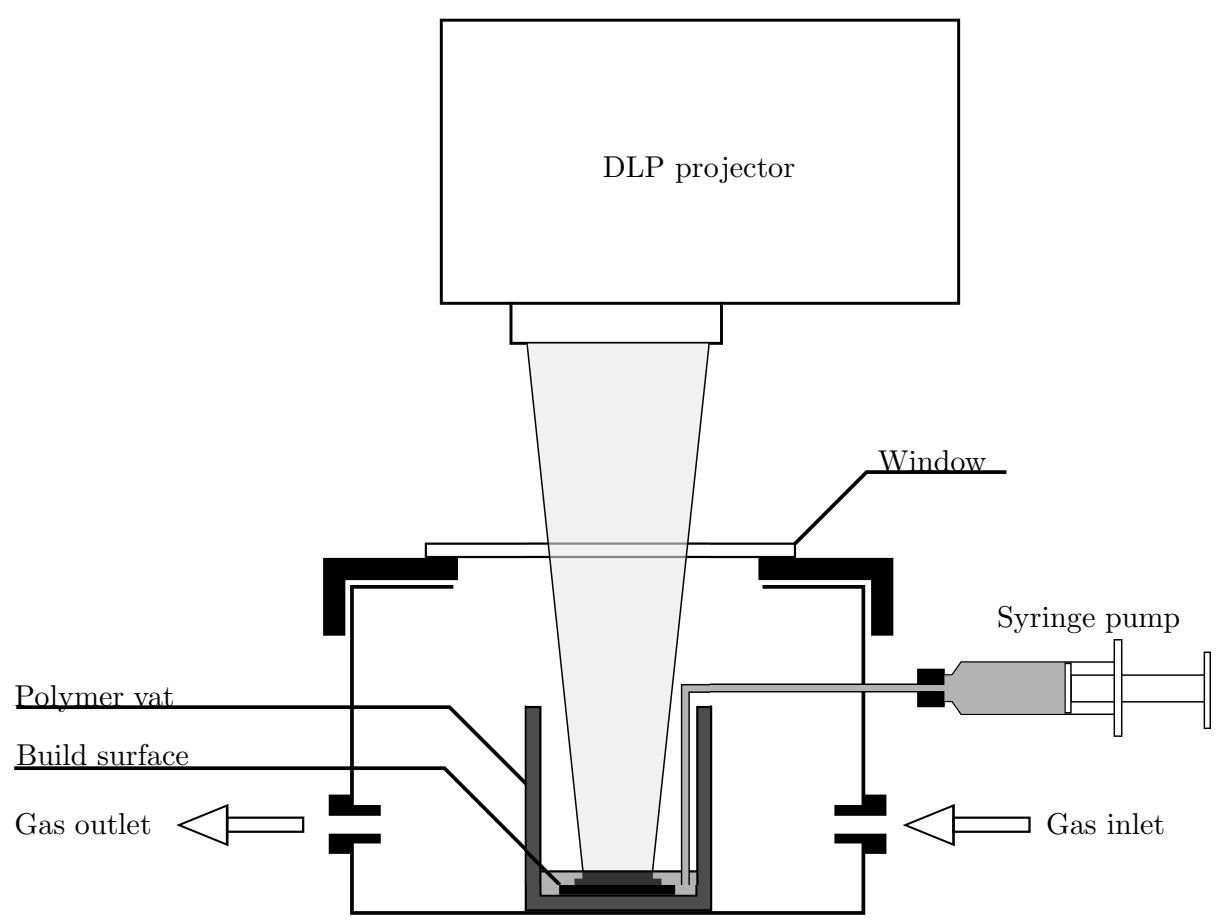

Figure B.2: Schematic diagram of the prototype free-surface additive manufacturing system

a uniform photopolymer layer was formed. The photopolymer was then drawn from the vat until the desired layer thickness was achieved. 


\title{
Appendix C
}

\section{Curriculum vitæ}

\author{
Name: $\quad$ Andrew Thomas Cullen \\ Post-Secondary The University of Western Ontario \\ Education and London, Ontario, Canada \\ Degrees: $\quad 2012-2016$ B.E.Sc. in Mechatronic Systems Engineering \\ Honours and NSERC \\ Awards: $\quad$ Canada Graduate Scholarship - Master's Program \\ $2017-2018$ \\ Province of Ontario Graduate Scholarship (declined) \\ $2017-2018$ \\ Province of Ontario Graduate Scholarship \\ $2016-2017$ \\ The University of Western Ontario \\ Dean's Honour List \\ $2013-2016$ \\ Related Work Teaching Assistant \\ Experience: The University of Western Ontario \\ $2016-2018$
}




\section{Patent Applications:}

- A. Price, A. Cullen, Method and System for Fabrication of Multilayered Conductive Polymer Structures, US Provisional Patent, serial no. 62/477,625, priority date 2017-03-28.

\section{Publications:}

- Cullen, A.T. \& Price, A.D. (2018). Digital light processing 3D printing of intrinsically conductive polymer structures. Synthetic Metals, 235, 34-41.

- Cullen, A.T. \& Price, A.D. (2017). Fabrication of multilayered conductive polymer structures via selective visible light photopolymerization. In Electroactive Polymer Actuators and Devices (EAPAD) 2017 (p. 101632N). Proc. SPIE. 